\title{
A high throughput screen for TMPRSS2 expression identifies FDA-approved and clinically advanced compounds that can limit SARS-CoV-2 entry
}

\section{Yanwen Chen}

Shanghai Jiao Tong University https://orcid.org/0000-0002-3467-2871

Travis Lear

University of Pittsburgh https://orcid.org/0000-0001-9156-0844

John Evankovich

University of Pittsburgh https://orcid.org/0000-0003-2070-0883

\section{Mads Larsen}

University of Pittsburgh

\section{Bo Lin}

University of Pittsburgh https://orcid.org/0000-0002-2108-2696

Irene Alfaras

University of Pittsburgh

Jason Kennerdell

University of Pittsburgh

\section{Laura Salminen}

University of Pittsburgh

\section{Daniel Camarco}

University of Pittsburgh

Karina Lockwood

University of Pittsburgh

Jie Liu

University of Pittsburgh

Michael Myerburg

University of Pittsburgh

John McDyer

University of Pittsburgh

Yuan Liu

University of Pittsburgh

\section{Toren Finkel}

University of Pittsburgh

Bill Chen ( $\square$ chenb@upmc.edu ) 


\section{Article}

Keywords: COVID-19, SARS-CoV-2, coronavirus, TMPRSS2, high throughput screen, clinical trial compounds

Posted Date: August 14th, 2020

DOI: https://doi.org/10.21203/rs.3.rs-48659/v1

License: (c) (1) This work is licensed under a Creative Commons Attribution 4.0 International License. Read Full License

Version of Record: A version of this preprint was published at Nature Communications on June 23rd, 2021. See the published version at https://doi.org/10.1038/s41467-021-24156-y. 
A high throughput screen for TMPRSS2 expression identifies FDA-approved and clinically advanced compounds that can limit SARS-CoV-2 entry

Yanwen Chen ${ }^{1,2^{*}}$, Travis B. Lear ${ }^{1,3,4^{*}}$, John W. Evankovich ${ }^{1,3^{*}}$, Mads B. Larsen ${ }^{1}$, Bo Lin ${ }^{1}$, Irene Alfaras ${ }^{1}$, Jason R. Kennerdell ${ }^{1}$, Laura Salminen ${ }^{1}$, Daniel P. Camarco', Karina C.

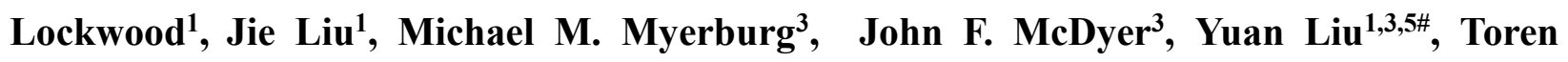

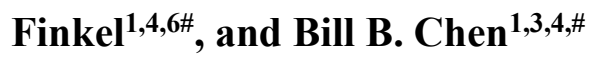

${ }^{1}$ Aging Institute, University of Pittsburgh/UPMC, Pittsburgh, PA 15219, USA

${ }^{2}$ Department of Gastroenterology, Ruijin Hospital, Shanghai Jiaotong University School of Medicine, Shanghai 200025, China

${ }^{3}$ Department of Medicine, Acute Lung Injury Center of Excellence, University of Pittsburgh, Pittsburgh, PA 15213, USA

${ }^{4}$ Vascular Medicine Institute, University of Pittsburgh, Pittsburgh, PA, 15213, USA

5 McGowan Institute for Regenerative Medicine, University of Pittsburgh, Pittsburgh, PA 15219, USA

${ }^{6}$ Department of Medicine, Division of Cardiology, University of Pittsburgh, Pittsburgh, PA 15213, USA

*These authors contributed equally to this work

Address Correspondence to:

Bill B. Chen, PhD \#

Toren Finkel, MD., PhD \#

Yuan Liu, PhD \#

University of Pittsburgh

Aging Institute, Department of Medicine

558 Bridgeside Point 1

100 Technology Drive

Pittsburgh, PA 15219

E-mail: chenb@upmc.edu, finkelt@pitt.edu, liuy13@upmc.edu 
SARS-CoV-2 (2019-nCoV) is the pathogenic coronavirus responsible for the global pandemic of COVID-19 disease. The Spike (S) protein of SARS-CoV-2 attaches to host lung epithelial cells through the cell surface receptor ACE2, a process dependent on host proteases including TMPRSS2. Here, we identified small molecules that can reduce surface expression of TMPRSS2 using a 2,700 FDA-approved or current clinical trial compounds. Among these, homoharringtonine and halofuginone appear the most potent agents, reducing endogenous TMPRSS2 expression at sub-micromolar concentrations. These effects appear to be mediated by a drug-induced alteration in TMPRSS2 protein stability. We further demonstrate that halofuginone modulates TMPRSS2 levels through proteasomal-mediated degradation that involves the E3 ubiquitin ligase component DDB1- and CUL4-associated factor 1 (DCAF1). Finally, cells exposed to homoharringtonine and halofuginone, at concentrations of drug known to be achievable in human plasma, demonstrated marked resistance to SARS-CoV-2 pseudoviral infection. Given the safety and pharmacokinetic data already available for the compounds identified in our screen, these results should help expedite the rational design of human clinical trials designed to combat COVID-19 infection. 


\section{Introduction}

SARS-CoV-2 is a coronavirus first described in Wuhan, China that shares many similarities with other pathogenic beta coronaviruses, including the SARS-CoV virus and the MERS coronavirus (1). After emergence of SARS-CoV, several groups identified the molecular and cellular pathways through which this virus attaches, enters, and replicates in host respiratory epithelial cells (2-4). To mediate cell entry, the SARS-CoV viral Spike (S) glycoprotein is recognized by the extracellular receptor angiotensin converting enzyme 2 (ACE2) on host respiratory epithelial cells $(5,6)$. A second critical process, $\mathrm{S}$ protein priming, is essential to complete viral entry and spread (7). S protein priming is a process wherein the spike protein is cleaved by host proteases. Two separate classes of proteases were shown to prime SARS-CoV S protein - endo-lysosomal proteases in the cathepsin family, and the plasma membrane associated protease, TMPRSS2 $(3,8)$. The viral entry mechanisms elucidated for SARS-CoV infection appear to be shared by SARSCoV-2 (9, 10). Additionally, SARS-CoV-2 uniquely possesses a "furin-like" cleavage site and is subject to processing during viral packaging by the intracellular proprotein convertase furin (1114).

Host-directed efforts to limit SARS-CoV-2 infection could logically entail strategies to manipulate either ACE2 or the S protein priming step. However, in animal models, reducing ACE2 activity appears to increase lung injury in response to SARS-CoV infection or sepsis $(6,15)$. In contrast, mice lacking TMPRSS2 exhibit no discernable basal phenotype (16), but demonstrate protection from acute SARS-CoV infection (3). These observations led us, and others $(9,17)$, to hypothesize that reducing or inhibiting TMPRSS2 may be an attractive strategy to mitigate SARS-CoV-2 pathogenicity. Given SARS-CoV-2's rapid spread, coupled with the high burden of acute respiratory failure and death, there is an urgent need for therapies. We believe the most rapid and effective near-term approach is to identify current FDA approved drugs that can be re-purposed to block viral entry and or spread. As such, we have executed a screen of approximately 2,700 FDAapproved or current clinical trial compounds to ascertain their ability to be repurposed to reduce TMPRSS2 abundance and prevent SARS-CoV-2 entry in lung epithelial cells. Additionally, we provide insight into the cellular regulation of TMPRSS2 - notably that it is targeted for E3 ligase driven ubiquitination and proteasomal degradation. 


\section{Result}

In order to assess agents that might alter TMPRSS2 expression, we first engineered full length human TMPRSS2 to express a C-terminal 11 amino acid tag (HiBiT), which produces a bioluminescent signal when combined with a complementary protein $(\mathrm{LgBiT})$ and a furimazine substrate (18). The HiBiT-tagged TMPRSS2 construct was expressed in human bronchial epithelial cells (Beas-2B) allowing us to detect either plasma membrane associated TMPRSS2, using bioluminescence associated with a non-lytic nano-luciferase (Nano-Luc) reagent or total TMPRSS2 expression, using a lytic luciferase agent (Fig. 1). Elements of the screen were optimized and miniaturized to a 384 -well format. We calculated the $Z$ ' score to be 0.38 , and thus suitable for high-throughput applications (fig. S1). We next screened a chemical library (FDAapproved and current clinical trial drugs) consisting of $\sim 2,700$ compounds for their capacity to decrease TMPRSS2-dependent Nano-Luc activity. We assayed the compounds effect on both the extracellular plasma membrane associated and total TMPRSS2-HiBiT signal using the non-lytic and lytic luciferase reagent. At a compound concentration of $10 \mu \mathrm{M}$, this screen identified $\sim 100$ well-characterized drugs that produced a $>50 \%$ reduction in either extracellular or total TMPRSS2-HiBiT signal (Fig. 2). A number of compounds were positive in both screens (Fig. 2c: intersection of purple and pink rectangles). In contrast, the agent nafamostat, a serine protease inhibitor, resulted in the opposite effect, as treatment with this agent potently increased TMPRSS2 expression (Fig. 2c). This compound is known to block TMPRSS2 enzymatic activity and thereby serve as a potential strategy to limit SARS-CoV and MERS acute infection of cells (19). A related compound, camostat, is also known to enzymatically inhibit TMPRSS2, and was recently shown to block acute SARS-CoV-2 infection (9), prompting the initiation of a clinical trial for this agent in COVID-19 patients (ClinicalTrials.gov Identifier: NCT04321096). However, our results suggest there might be a feedback mechanism through which TMPRSS2 enzymatic activity regulates overall levels of the protease. As such, these observations serve as a potentially cautionary note for using TMPRSS2 enzymatic inhibitors as a means to reduce coronavirus infections $(9,19)$.

The most potent compounds (24 in total) were subsequently cherry-picked and tested extensively in dose-course studies for their effect on the extracellular and intracellular TMPRSS2-HiBiT signal 
in airway epithelial cells (Fig. 3 and fig. S2). These agents were also directly assessed by Western blot analysis for TMPRSS2-HiBiT protein expression (fig. S3). An assessment of the compound's toxicity, as measured by the drug's effect on cell number (using CellTiter-Glo 2.0) was also determined, so as to give an estimate of each compounds' potential therapeutic index (Fig. 3 and fig. S2). As noted, many agents, including homoharringtonine (approved for chronic myeloid leukemia), halofuginone (in clinical trials for scleroderma) and cilnidipine (a calcium-channel blocker, antihypertensive agent approved in Asia and in some European countries), were effective in reducing extracellular TMPRSS2 expression at sub-micromolar concentrations. In general, these agents were also effective in reducing total TMPRSS2 as well, although for certain agents (e.g. cilnidipine) this required a substantially higher concentration of drug.

The clinical utility of any agent identified ultimately depends on the ability of the potential therapeutic compound to reach presumed therapeutic levels in patients. As such, we compared the measured IC50 from our screen to the known pharmacokinetic properties of the identified agents. While we would ideally have preferred knowing the concentration of each drug in the presumptive target organ (e.g. lung), such information is not currently publicly available for many of these agents. As such, as an approximation, we leveraged the known concentration of each of these compounds in human plasma. From this exercise, homoharringtonine and halofuginone emerged as the candidates most likely to be clinically effective. We next sought to confirm that the reduction in the Nano-Luciferase signal observed in our screen by these two agents was not cell-type specific, dependent on the HiBiT tag, or a transcriptional effect of these compounds on the heterologous CMV promoter used to drive expression of our HiBiT-tagged TMPRSS2 construct. Using the same CMV promoter, we transiently expressed either a V5-tagged TMPRSS2 or a V5-tagged LacZ protein in the mouse respiratory epithelial cell line MLE-12. As noted in Figure 4a, both agents seem to selectively reduce TMPRSS2 expression in cells.

We then asked whether these compounds were effective in reducing endogenous TMPRSS2 expression. We chose the intestinal epithelial cell line Caco-2, since these cells are known to express high levels of TMPRSS2 and to be permissive for both SARS-CoV and SARS-CoV-2 infection $(9,20)$. Moreover, evidence suggests that the human intestinal tract may be an alternative entry point for coronaviruses (21). Treatment of Caco-2 cells for 18 hours with either 
homoharringtonine or halofuginone resulted in a marked reduction in endogenous TMPRSS2 protein expression (Fig. 4b, c). As expected, the observed decrease in TMPRSS2 protein expression was not a consequence of a drug-induced reduction in TMPRSS2 transcription, which if anything, modestly increased with compound treatment (fig. S4). We also noted qualitatively similar decreases in TMPRSS2 protein levels in homoharringtonine or halofuginone-treated Calu3 cells, a human lung cancer cell line that is also permissive for SARS-CoV-2 infection (9) (fig. $\mathrm{S} 5 \mathrm{a}, \mathrm{b})$. A time course demonstrated that the effects of drug treatment were relatively rapid, with a substantial reduction in TMPRSS2 expression evident within three hours (Fig. 4d, Fig. S5c). Similarly, levels of TMPRSS2 returned close to baseline roughly six hours after drug removal (Fig. 4e, Fig. S5d).

While homoharringtonine is a relatively toxic agent with an adverse side effect profile $(22,23)$, halofuginone, has a very mild toxicity profile and is generally well tolerated $(24,25)$. Given the potential safety advantages of halofuginone, we therefore sought to gain additional understanding of how this agent modifies TMPRSS2 expression. Given the rapid decay of TMPRSS2 upon halofuginone treatment, we initially focused our efforts on determining the means of protein degradation. While the lysosomal inhibitor Bafilomycin A1 was without effect, the effects of halofuginone on TMPRSS2 protein levels were largely abrogated in the presence of the proteasomal inhibitor carfilzomib (Fig 5a). This argues that TMPRSS2 likely undergoes clearance through the ubiquitin-proteasomal system (UPS). To gain further mechanistic insight into this process, we employed Beas-2B cells stably expressing TMPRSS2-Hibit to perform a highthroughput screen using siRNAs targeting $\sim 800$ components of the UPS including siRNAs targeting ubiquitin, proteasome subunits, E1, E2, E3, deubiquitinases (DUBs), and E3 ligases. Knockdown of ubiquitin or proteasome subunits increased TMPRSS2 HiBiT signal robustly, consistent with our assumption that the protein is subject to UPS-mediated turnover (Fig 5b-c). We also observed that knockdown of the E3 ligase subunit DCAF1 increased TMPRSS2 abundance (red dots) (Fig. 5b-c). Overexpression of DCAF1 decreased TMPRSS2-V5 protein in total cell lysate, and also increased poly-ubiquitination of TMPRSS2, suggesting a role of this E3 component in TMPRSS2 ubiquitination and half-life (Fig. 5d). Overexpression of DCAF1 also dose-dependently decreased endogenous TMPRSS2 (Fig. 5e). To further establish the mechanism by which halofuginone modulates TMPRSS2 stability, we performed siRNA-mediated 
knockdown of DCAF1 in the setting of halofuginone treatment. In control knockdown cells, halofuginone reduced TMPRSS2 expression without altering the levels of another cell surface protein, E-cadherin. In cells subjected to DCAF1 siRNA mediated knockdown (using two separate siRNAs), we noted an increase in basal levels of TMPRSS2. Of note, knockdown of DCAF1 knockdown abrogated the effects of halofuginone on TMPRSS2 protein levels (Fig. 5f). We next took advantage of the observation that lysine residues in target proteins are often the site of E3mediated ubiquitination (26). TMPRSS2 contains four lysine residues on its intracellular domain, with three lysines closely clustered (K80, K82 and K83). In contrast to the wild-type protein, or to a site directed mutant in which only two lysines were altered, a site-directed mutant of TMPRSS2 in which all three lysines were converted to arginine was resistant to the effects of halofuginone (Fig. 5g). This suggests that DCAF1 is a potential E3 ligase subunit that regulates TMPRSS2 stability through ubiquitination. Of note, halofuginone has also been postulated to act as a glutamyl-prolyl-tRNA synthetase inhibitor, inhibiting translation of a subset of proteins (27). While halofuginone does not exhibit strict substrate specificity, it has been proposed that halofuginone reduces the rate of translation of "proline-rich" proteins. In that regard, we note that TMPRSS2 sequence contains 7\% proline residues. Indeed, we observed that halofuginone can inhibit the in vitro and in vivo synthesis of TMPRSS2 in a proline-dependent fashion (fig. S6a, b). As such, it seems likely that halofuginone can affect both the synthesis and degradation of TMPRSS2.

We next asked whether the agents we have identified could effectively limit viral infection. Given the biosafety issues of working with live SARS-CoV-2, we addressed this scientific question by creating a pseudovirus, which incorporated the SARS-CoV-2 Spike protein. For the sake of viral entry, this pseudovirus faithfully recapitulates COVID-19 infection. Of note, this strategy has been employed recently by other groups $(9,28,29)$. In our case, we also molecularly tagged the Spike protein with the HiBiT sequence to allow rapid and sensitive detection of viral infection. The strategy employed is diagramed in Figure 6a. We first assessed the ability of our SARS-CoV-2 pseudovirus to infect various cell lines, and as previously noted, both Caco-2 and Calu-3 cells appeared to be highly permissive (Fig 6b), consistent with the known high level expression of both ACE2 and TMPRSS2 in these cell lines $(9,20,30)$. We then asked whether homoharringtonine or halofuginone were biologically active in reducing SARS-CoV-2 infection. Remarkably, both 
agents were able to affect a $50 \%$ reduction in the level of viral infection at concentration $\sim 30 \mathrm{nM}$ (Fig. 6c, d). Similar, albeit slightly less potent effects, were also seen in Calu-3 cells (fig. S7a, b). We noted that other agents identified in our screen including cilnidipine, dasatinib and venetoclax were also effective in reducing viral entry (fig. S7c, d). Since our pseudovirus also encodes for a GFP reporter, we also assessed this parameter in pseudovirally-infected cells. As noted, both homoharringtonine and halofuginone markedly reduced levels of GFP expression (Fig. 6e), again consistent with their ability to inhibit viral entry. We also tested a separate pseudovirus which encodes for a luciferase-based reporter and showed a similar dose-dependent reduction of the luciferase signal using both homoharringtonine and halofuginone (Fig. S8a, b). In addition, we noted that combined treatment with homoharringtonine and halofuginone was more effective than either agent alone (Fig. S8c).

Finally, given that each permissive cell line has a different reliance on TMPRSS2- versus endosomal-mediated viral entry pathways, we sought to ascertain the effects of our identified compounds on the most clinically relevant cell type, namely primary human respiratory epithelial cells. These primary cells were obtained from normal human lungs under a protocol approved by our Institutional Review Board (IRB) and maintained at an air-liquid interface. In culture, these cells retain features of their normal apical-basal polarity and exhibit the expected bronchial epithelial mucociliary phenotype $(31,32)$. Similar to what we observed in immortalized cell lines, homoharringtonine and halofuginone were also effective in blocking SARS-CoV-2 pseudoviral entry in these primary human bronchial epithelial cells (Fig. 6f, g). Lastly, we confirmed the role of DCAF1 in regulating the beneficial effects of halofuginone. Of note, DCAF1 knockdown significantly blocked the observed halofuginone-mediated reduction in SARS-CoV-2 pseudoviral entry (Fig. 6h). 


\section{Discussion}

By executing an unbiased small molecule screen, we have identified a number of compounds that are currently in active clinical trials or are FDA-approved that can reduce TMPRSS2 expression (Table I). Using the biological IC50 for inhibiting pseudoviral entry, several of these candidates would appear to be clinically viable. The agents identified in our screen may be effective alone, or in combination with each other (Fig. S8c). In addition, as noted initially, SARS-CoV-2 can enter cells through TMPRSS2-mediated pathways, or can employ endolysosomal proteases such as cathepsin $\mathrm{L}$ to cleave the $\mathrm{S}$ protein and gain cell entry $(8,33)$. If agents such as chloroquine, which modify endosomal $\mathrm{pH}$, are ultimately determined to be effective for COVID-19 infections, we hypothesize that they would likely be synergistic with agents identified here that inhibit TMPRSS2-mediated pathways (fig. S11). Our approach, would also appear to complement strategies that directly target viral replication such as remdesivir (34).

It should be noted that our two most promising leads, homoharringtonine and halofuginone, have been noted to have anti-viral activity (35-37), although the precise mechanism for these effects were not previously determined. Our in vitro data would indicate that TMPRSS2 reduction occurs at concentrations that are achievable with the approved dosing in humans (see Table 1). As noted, homoharringtonine is a chemotherapeutic agent that can trigger myelosuppression $(22,23)$, while halofuginone is generally better tolerated $(24,25)$. As such, we concentrated our mechanistic studies on the latter, likely more clinically viable agent, demonstrating that TMPRSS2 is targeted for proteasomal-mediated degradation. We have previously described an unbiased siRNAmediated strategy to rapidly identify which of the more than $600 \mathrm{E} 3$ ubiquitin ligases are relevant for a given target's half-life (38). Using that platform, we were able to identify DCAF1 as an important regulator of TMPRSS2 stability. Of note, knockdown of DCAF1 raised the basal level of TMPRSS2. More importantly, knockdown of DCAF1 abrogated the ability of halofuginone to trigger a decline in TMPRSS2 levels. It, similarly, reduced the ability of halofuginone to block SARS-CoV2 pseudoviral entry. 
These results suggest that halofuginone catalyzes a reduction of TMPRSS2 protein levels through a DCAF1-dependent pathway. This is further supported by the observation that altering a cluster of critical lysine residues on TMPRSS2's cytoplasmic domain abrogates the effects of halofuginone. Our preliminary data suggests that halofuginone does not inhibit TMPRSS2 catalytic activity (Fig. S9) nor directly binds to either TMPRSS2 or DCAF1 (Fig. S10) suggesting the drug effects may occur indirectly, perhaps by triggering a post-translational modification of TMPRSS2 that favors an enhanced interaction with DCAF1. One such possible post-translational modification comes from previous observations that DCAF1 binding to a substrate can be triggered my mono-methylation of the substrate thereby creating a methyl-degron (39). Whether or not halofuginone can catalyze this or other modifications will require additional study. Of note, halofuginone has also been postulated to act as a glutamyl-prolyl-tRNA synthetase inhibitor, inhibiting translation of a subset of proteins (27). Our data suggests this mechanism may be operative here as well. As such, it appears possible that halofuginone could affect both the translation and the post-translational stability of TMPRSS2.

While we were focusing on TMPRSS2 in this study, recent work has demonstrated that several other host proteases can perform the critical $\mathrm{S}$ protein priming step. Notably, SARS-CoV-2 contains an extra "RRAR" amino acid sequence in the S1/S2 domain, making it susceptible to cleavage by the host protease furin during viral packaging (11-14). Furin is a highly expressed proprotein convertase that performs vital cellular functions, and the presence of this furin-like cleavage domain in other viruses is associated with increased pathogenicity and neurotropism (4042). Future studies are needed to determine whether halofuginone or other promising hits from this study can also reduce furin, or other key host proteases, involved in SARS-CoV2 entry.

Finally, while our results focused on strategies that alter the post-translational stability of TMPRSS2, it should be noted that TMPRSS2 expression is known to be transcriptionally regulated by androgens (43). It is intriguing to speculate whether this may translate into a higher basal level of TMPRSS2 in men, and in turn, whether this higher level of TMPRSS2 expression can partially explain why men appear to be at significantly higher risk for mortality and complications following COVID-19 infection (44). Of note, individuals who have inherited a non-coding SNP that only modestly increases the expression of TMPRSS2, appear to be at a significant elevated risk for developing more severe viral infections (45). As such, we believe that agents identified here, that 
reduce TMPRSS2 expression, represent a rational approach to modify the clinical course of COVID-19, and potentially future related viral pandemics.

\section{ACKNOWLEDGMENTS}

This work was supported by NIH grants to BBC (5R35HL139860 and 5R01HL133184), YL (5R01HL142777), JWE (1K08HL144820), TBL (T32 HL110849), JFM (R01 133184) and TF (1R01 HL142663, 1R01HL142589 and P30 AG024827), the University of Pittsburgh Aging Institute seed fund to $\mathrm{BBC}, \mathrm{TF}$ and YL and a grant from Jewish Healthcare Foundation (TF). We thank Cystic Fibrosis Research Development Program at the University of Pittsburg, School of Medicine, Pittsburgh, PA for providing HBE cells.

\section{AUTHOR CONTRIBUTIONS}

$\mathrm{BBC}, \mathrm{TF}, \mathrm{YL}$ designed and directed the study. YC, TBL, JWE, BBC, TF analyzed the data, prepared the figures, and wrote the manuscript. YC, TBL, JWE, MBL, IA, BL, JRK, LAS, KCL, JL, YL and BBC performed all experiments. MMM and JFM provided help with the human bronchial epithelial cells. DPC assisted with high-throughput screening. BBC, YL, JWE, and TF provided funding for the studies.

\section{DATA AVAILABILITY}

The datasets generated during and/or analyzed during the current study are available from the corresponding author on reasonable request

Supplementary Materials

MATERIALS and METHODS

Figs S1 to S11 
References:

1. Y. Yin, R. G. Wunderink, MERS, SARS and other coronaviruses as causes of pneumonia. Respirology 23, 130-137 (2018).

2. I. Glowacka et al., Evidence that TMPRSS2 activates the severe acute respiratory syndrome coronavirus spike protein for membrane fusion and reduces viral control by the humoral immune response. J Virol 85, 4122-4134 (2011).

3. N. Iwata-Yoshikawa et al., TMPRSS2 Contributes to Virus Spread and Immunopathology in the Airways of Murine Models after Coronavirus Infection. J Virol 93, (2019).

4. H. Hofmann, S. PohImann, Cellular entry of the SARS coronavirus. Trends Microbiol 12, 466-472 (2004).

5. H. P. Jia et al., ACE2 receptor expression and severe acute respiratory syndrome coronavirus infection depend on differentiation of human airway epithelia. J Virol 79, 14614-14621 (2005).

6. K. Kuba et al., A crucial role of angiotensin converting enzyme 2 (ACE2) in SARS coronavirusinduced lung injury. Nat Med 11, 875-879 (2005).

7. M. A. Tortorici, D. Veesler, Structural insights into coronavirus entry. Adv Virus Res 105, 93-116 (2019).

8. G. Simmons et al., Inhibitors of cathepsin L prevent severe acute respiratory syndrome coronavirus entry. Proc Natl Acad Sci U S A 102, 11876-11881 (2005).

9. M. Hoffmann et al., SARS-CoV-2 Cell Entry Depends on ACE2 and TMPRSS2 and Is Blocked by a Clinically Proven Protease Inhibitor. Cell, (2020).

10. D. Wrapp et al., Cryo-EM structure of the 2019-nCoV spike in the prefusion conformation. Science 367, 1260-1263 (2020).

11. J. Shang et al., Cell entry mechanisms of SARS-CoV-2. Proc Natl Acad Sci U S A 117, 11727-11734 (2020).

12. J. A. Jaimes, J. K. Millet, G. R. Whittaker, Proteolytic Cleavage of the SARS-CoV-2 Spike Protein and the Role of the Novel S1/S2 Site. iScience 23, 101212 (2020).

13. B. Coutard et al., The spike glycoprotein of the new coronavirus 2019-nCoV contains a furin-like cleavage site absent in CoV of the same clade. Antiviral Res 176, 104742 (2020).

14. P. Anand, A. Puranik, M. Aravamudan, A. J. Venkatakrishnan, V. Soundararajan, SARS-CoV-2 strategically mimics proteolytic activation of human $\mathrm{ENaC}$. elife 9, (2020).

15. Y. Imai et al., Angiotensin-converting enzyme 2 protects from severe acute lung failure. Nature 436, 112-116 (2005).

16. T. S. Kim, C. Heinlein, R. C. Hackman, P. S. Nelson, Phenotypic analysis of mice lacking the Tmprss2-encoded protease. Mol Cell Biol 26, 965-975 (2006).

17. L. W. Shen, H. J. Mao, Y. L. Wu, Y. Tanaka, W. Zhang, TMPRSS2: A potential target for treatment of influenza virus and coronavirus infections. Biochimie 142, 1-10 (2017).

18. M. Soave, B. Kellam, J. Woolard, S. J. Briddon, S. J. Hill, NanoBiT Complementation to Monitor Agonist-Induced Adenosine A1 Receptor Internalization. SLAS Discov 25, 186-194 (2020).

19. M. Yamamoto et al., Identification of Nafamostat as a Potent Inhibitor of Middle East Respiratory Syndrome Coronavirus S Protein-Mediated Membrane Fusion Using the SplitProtein-Based Cell-Cell Fusion Assay. Antimicrob Agents Chemother 60, 6532-6539 (2016).

20. J. Cinatl, Jr. et al., Infection of cultured intestinal epithelial cells with severe acute respiratory syndrome coronavirus. Cell Mol Life Sci 61, 2100-2112 (2004).

21. J. Zhou et al., Human intestinal tract serves as an alternative infection route for Middle East respiratory syndrome coronavirus. Sci Adv 3, eaao4966 (2017).

22. V. Gandhi, W. Plunkett, J. E. Cortes, Omacetaxine: a protein translation inhibitor for treatment of chronic myelogenous leukemia. Clin Cancer Res 20, 1735-1740 (2014). 
23. S. Lü, J. Wang, Homoharringtonine and omacetaxine for myeloid hematological malignancies. J Hematol Oncol 7, 2 (2014).

24. M. Pines, D. Snyder, S. Yarkoni, A. Nagler, Halofuginone to treat fibrosis in chronic graft-versushost disease and scleroderma. Biol Blood Marrow Transplant 9, 417-425 (2003).

25. M. Pines, I. Spector, Halofuginone - the multifaceted molecule. Molecules 20, 573-594 (2015).

26. A. Hershko, A. Ciechanover, The ubiquitin system. Annu Rev Biochem 67, 425-479 (1998).

27. T. L. Keller et al., Halofuginone and other febrifugine derivatives inhibit prolyl-tRNA synthetase. Nat Chem Biol 8, 311-317 (2012).

28. X. Ou et al., Characterization of spike glycoprotein of SARS-CoV-2 on virus entry and its immune cross-reactivity with SARS-CoV. Nat Commun 11, 1620 (2020).

29. A. C. Walls et al., Structure, Function, and Antigenicity of the SARS-CoV-2 Spike Glycoprotein. Cell, (2020).

30. M. Kawase, K. Shirato, L. van der Hoek, F. Taguchi, S. Matsuyama, Simultaneous treatment of human bronchial epithelial cells with serine and cysteine protease inhibitors prevents severe acute respiratory syndrome coronavirus entry. J Virol 86, 6537-6545 (2012).

31. M. M. Myerburg, P. R. Harvey, E. M. Heidrich, J. M. Pilewski, M. B. Butterworth, Acute regulation of the epithelial sodium channel in airway epithelia by proteases and trafficking. Am J Respir Cell Mol Biol 43, 712-719 (2010).

32. A. T. Lennox et al., ATP12A promotes mucus dysfunction during Type 2 airway inflammation. Sci Rep 8, 2109 (2018).

33. I. C. Huang et al., SARS coronavirus, but not human coronavirus NL63, utilizes cathepsin L to infect ACE2-expressing cells. The Journal of biological chemistry 281, 3198-3203 (2006).

34. H. Sivaraman, S. Y. Er, Y. K. Choong, E. Gavor, J. Sivaraman, Structural Basis of the SARS-CoV2/SARS-CoV Receptor Binding and Small-Molecule Blockers as Potential Therapeutics. Annu Rev Pharmacol Toxicol, (2020).

35. P. I. Andersen et al., Novel Antiviral Activities of Obatoclax, Emetine, Niclosamide, Brequinar, and Homoharringtonine. Viruses 11, (2019).

36. H. J. Dong et al., The Natural Compound Homoharringtonine Presents Broad Antiviral Activity In Vitro and In Vivo. Viruses 10, (2018).

37. J. Hwang, A. Jiang, E. Fikrig, A potent prolyl tRNA synthetase inhibitor antagonizes Chikungunya and Dengue viruses. Antiviral Res 161, 163-168 (2019).

38. Y. Chen et al., A small molecule NRF2 activator BC-1901S ameliorates inflammation through DCAF1/NRF2 axis. Redox Biol 32, 101485-101485 (2020).

39. Ji M. Lee et al., EZH2 Generates a Methyl Degron that Is Recognized by the DCAF1/DDB1/CUL4 E3 Ubiquitin Ligase Complex. Molecular cell 48, 572-586 (2012).

40. X. Sun, L. V. Tse, A. D. Ferguson, G. R. Whittaker, Modifications to the hemagglutinin cleavage site control the virulence of a neurotropic H1N1 influenza virus. J Virol 84, 8683-8690 (2010).

41. G. Izaguirre, The Proteolytic Regulation of Virus Cell Entry by Furin and Other Proprotein Convertases. Viruses 11, (2019).

42. J. Cheng et al., The S2 Subunit of QX-type Infectious Bronchitis Coronavirus Spike Protein Is an Essential Determinant of Neurotropism. Viruses 11, (2019).

43. B. Lin et al., Prostate-localized and androgen-regulated expression of the membrane-bound serine protease TMPRSS2. Cancer Res 59, 4180-4184 (1999).

44. G. Grasselli et al., Baseline Characteristics and Outcomes of 1591 Patients Infected With SARSCoV-2 Admitted to ICUs of the Lombardy Region, Italy. Jama, (2020).

45. Z. Cheng et al., Identification of TMPRSS2 as a Susceptibility Gene for Severe 2009 Pandemic A(H1N1) Influenza and A(H7N9) Influenza. J Infect Dis 212, 1214-1221 (2015). 
46. J. Nemunaitis et al., Pharmacokinetic study of omacetaxine mepesuccinate administered subcutaneously to patients with advanced solid and hematologic tumors. Cancer Chemother Pharmacol 71, 35-41 (2013).

47. M. J. de Jonge et al., Phase I and pharmacokinetic study of halofuginone, an oral quinazolinone derivative in patients with advanced solid tumours. Eur J Cancer 42, 1768-1774 (2006).

48. J. Lee et al., Evaluation of the pharmacokinetic and pharmacodynamic drug interactions between cilnidipine and valsartan, in healthy volunteers. Drug Des Devel Ther 8, 1781-1788 (2014).

49. Y. Ishida et al., Pharmacokinetics and pharmacodynamics of dasatinib in the chronic phase of newly diagnosed chronic myeloid leukemia. Eur J Clin Pharmacol 72, 185-193 (2016).

50. S. K. Agarwal, B. Hu, D. Chien, S. L. Wong, A. H. Salem, Evaluation of Rifampin's Transporter Inhibitory and CYP3A Inductive Effects on the Pharmacokinetics of Venetoclax, a BCL-2 Inhibitor: Results of a Single- and Multiple-Dose Study. J Clin Pharmacol 56, 1335-1343 (2016).

51. R. Jafari et al., The cellular thermal shift assay for evaluating drug target interactions in cells. Nat Protoc 9, 2100-2122 (2014). 


\section{Figure Legends:}

Figure 1: Schematic of TMPRSS2-HiBiT detection for high throughput screen. (A) The split nano-luciferase components $\mathrm{LgBiT}$ and $\mathrm{HiBiT}$ can interact to form a functional enzyme that generates luminescence. (B) Human TMPRSS2 cDNA was C-terminally tagged with a HiBiT sequence on a domain that is extracellular when TMPRSS2 is present in the plasma membrane. (C) The TMPRSS2-HiBiT construct was expressed in human airway cells where it exists in an intracellular pool and a plasma membrane-associated pool. (D) Non-lytic extracellular HiBiT detection results in LgBiT-HiBiT complementation solely with the pool of plasma membrane localized TMPRSS2. Following extracellular HiBiT detection, cells are lysed and the total TMPRSS2- HiBiT is then quantified.

Figure 2: Screening of airway cells with a FDA-approved or clinically active compound library for agents that reduce TMPRSS2-HiBiT levels. (A) HTS results from the non-lytic extracellular HiBiT detection of TMPRSS2-HiBiT. The top 20 compounds are specified. (B) Lytic HiBiT detection reflecting total cellular TMPRSS2-HiBiT; top 20 compound hits are listed. (C) Scatterplot of hit compounds from both screens. Compounds that reduce membrane TMPRSS2HiBiT signal (non-lytic extracellular HiBiT detection) are shown in pink, compounds that reduce total TMPRSS2-HiBiT signal (lytic HiBiT detection) are shown in purple. Some individual compounds are specified.

Figure 3: Determination of the potency for a subset of identified compounds. A selection of six of the most promising drugs were assessed for their activity (IC50) to inhibit TMPRSS2 expression extracellularly (first column) or to inhibit total TMPRSS2 (middle column). An assessment of cellular toxicity for each compound (CellTiter-Glo) is shown in the last column. The remaining activity profiles for other identified agents are found in the supplementary figures. data are mean $+/$ - SEM $(n=4)$.

Figure 4: Homoharringtonine and halofuginone potently reduce TMPRSS2 protein levels. (A) Immunoblot data from MLE-12 cells co-expressing LacZ-V5 and TMPRSS2-V5 treated with homoharringtonine (HHT) or halofuginone (HFG) at the indicated concentrations for $18 \mathrm{hr}$. (B-C) Immunoblot analysis of endogenous TMPRSS2 protein level in Caco-2 cells treated for $18 \mathrm{hr}$ with HHT (B) or HFG (C). TMPRSS2 densitometry is shown, data are mean +/- SEM (n=3). (D) Timecourse treatment of HFG-treated Caco- 2 cells $(3 \mu \mathrm{M})$. TMPRSS2 densitometry is shown, data are mean + /- SEM ( $\mathrm{n}=3$ ). (E) Immunoblot analysis of Caco-2 cells treated with HFG for $18 \mathrm{hr}$ prior to removing the drug, adding fresh media, and then analyzing the protein recovery time course. (All TMPRSS2 densitometry that is shown represents mean $+/-$ SEM $(n=3)$. Actin is shown as a loading control. NS, $\mathrm{P}>0.05$; *, $\mathrm{P}<0.05$; **, $\mathrm{P}<0.01$; ***, $\mathrm{P}<0.001$; ****, $\mathrm{P}<0.0001$ relative to 0 time point or control, or as indicated by one-way ANOVA with Dunnett's test of multiple comparisons (B-E). 
Figure 5: TMPRSS2 is degraded through ubiquitin proteasome system, which is required for HFG efficacy. (A) Immunoblotting assay of Caco-2 cells treated with HFG in combination with carfilzomib (CFZ) or bafilomycin A1 (BafA1) and probed for TMPRSS2. (B-C) TMPRSS2HiBiT signal was measured following siRNA knockdown of ubiquitination-related machinery. (B) Ubiquitination siRNA library screening results ordered by increase in TMPRSS2-HiBiT signal. The E3 ligase DCAF1 was detected as a top hit. (C). Volcano plot of TMPRSS2-HiBiT signal screening with Ubiquitination siRNA library from $n=3$ independent screening assays. Statistical significance is plotted against $\log 2$-fold change in TMPRSS2-HiBiT signal. Top hits are annotated. (D) DCAF1 co-expression increases TMPRSS2 ubiquitination. (E) Immunoblot analysis of Beas2B cells with increasing expression of DCAF1, TMPRSS2 densitometry shown represents mean + - SEM (n=3). (F) Immunoblot analysis of Beas-2B cells with control or DCAF1 siRNA treatment followed by HFG treatment. TMPRSS2 densitometry represent mean -/+ SEM $(n=3)$. (G) TMPRSS2 intracellular lysines were assayed for their responsiveness to HFG, data represent mean $+/$ - SEM $(n=3)$. Actin is shown as a loading control throughout, and E-cadherin is added as an additional plasma membrane loading control. NS, $\mathrm{P}>0.05$; *, $\mathrm{P}<0.05 ; * *, \mathrm{P}<0.01 * * *, \mathrm{P}<$ 0.001 ; ***, $\mathrm{P}<0.0001$ relative to control, or as indicated by one-way ANOVA with Dunnett's test of multiple comparisons (E-F), or two-way unpaired t-test (G).

Figure 6: Agents that reduce TMPRSS2 expression markedly inhibit SARS-CoV-2 pseudoviral infection. (A) Schematic of pseudoviral construction and assay. The S protein of SARS-CoV-2 was C-terminally tagged with HiBiT. (B) Level of viral transduction in various cell lines plotted on a logarithmic scale. Calu-3 and Caco-2 cells had the highest observed rates of infection. (C-D) Effects of increasing concentrations of homoharringtonine (HHT) (C) or halofuginone (HFG) (D) on SARS-CoV-2 pseudoviral infection. (E) Effects of HHT or HFG on pseudoviral-mediated GFP expression, determined by immunostaining. (F-G) SARS-CoV-2 pseudoviral infection of primary human bronchial epithelial cells in the presence of increasing concentrations of HHT (F) and HFG (G). (H) SARS-CoV-2 pseudoviral infection of Caco-2 cells transfected with DCAF1 siRNA along with HFG treatment (100nM). All SARS-CoV-2 pseudoviral data is corrected to cell number as determined by CellTiter-Glo. **, $\mathrm{P}<0.01 ; * * *, \mathrm{P}$ $<0.001$; ***, $\mathrm{P}<0.0001$ relative to 0 time point or control, or as indicated by one-way ANOVA with Dunnett's test of multiple comparisons (F-G), or a two-way ANOVA with Tukey's test of multiple comparisons $(\mathrm{H})$

Table 1: Identified agents' current clinical indications, human pharmacokinetic properties (46-50) and estimated IC50 for inhibiting viral entry into Caco-2 cells. 
Supplemental Materials:

MATERIALS AND METHODS

Materials-

High-Capacity cDNA Reverse Transcription Kit (4368814) and SYBR Green PCR Master Mix (4364344) were from Applied Biosystems. BEAS-2B (CRL-9609), Caco-2 (HTB-37), Calu-3 (HTB-55), MLE-12 (CRL-2110) and Vero (CCL-81) cells were all obtained from ATCC. Easy Prep RNA Miniprep Plus Kit (R01-04) was from Bioland Scientific. DC Protein Assay Reagent A/B/S (500-0113/ 0114/ 0115) was from BioRad. DMEM/F-12 (11320082), EMEM (670086), Fetal Bovine Serum (26140079) and Opti-MEM I Reduced Serum Medium (31985062) were from Gibco. Amaxa Nucleofector II was from Lonza. Anti- $\beta$-actin antibody (MA5-15739), Countess II Automated Cell Counter (AMQAX1000) and Lipofectamine 3000 Transfection Reagents (L3000015) were from Invitrogen. HIV-1 Gag p24 DuoSet ELISA (DY7360-05) was from R\&D Systems. SARS-CoV-2 (2019-nCoV) Spike ORF mammalian expression plasmid (Codon Optimized) (VG40589-UT) was from SinoBiological. The following reagent was obtained through the NIH AIDS Reagent Program, Division of AIDS, NIAID, NIH: HIV-1 pNL4-3 $\Delta$ Env Vpr Luciferase Reporter Vector (pNL4-3.Luc.R-E-) from Dr. Nathaniel Landau. pSF-CMV-VSVG (OG592) VSV G Expression Plasmid (SnapFast Pro) (OG592) was from Oxgene. AsiSI (R0630), Eco53kI (R0116S), PmeI (R0560), Quick Ligation Kit (M2200) were from New England Biolabs (NEB). Mouse monoclonal anti-V5 Tag (R960-25) and TMPRSS2 antibody (PA5-83286) were from Thermo Fisher. X-tremeGENE HP DNA Transfection Reagent (6366244001) and XtremeGENE siRNA Transfection Reagent (4476115001) were from Sigma-Aldrich. Lenti-X GoStix Plus (631280) were from Takara. Venetoclax (HY-15531) and Homoharringtonine (HY14944) were from MedChemExpress. Halofuginone (S8144) and Cilnidipine (S1293) were from 
SelleckChem. Bafilomycin A1 (11038), Carfilzomib (17554), Dasatinib (11498), and Verteporfin (17334) were from Cayman Chemical. Antibody against E-cadherin (G-10, sc-8426) was from Santa Cruz Biotechnologies. MG132 (F1100) was from UBPBio. CellTiter-Glo 2.0 Cell Viability Assay (G9243), Nano-Glo HiBiT Lytic Detection System (N3040), Nano-Glo HiBiT Extracellular Detection System (N2421), Nano-Glo HiBiT Blotting System (N2410), HiBiT CMV-neo Flexi Vectors (N2401, N2391) were from Promega.

Cell Culture- Beas-2b and MLE-12 cells from ATCC were cultured in HITES media supplemented with 10\% fetal bovine serum (FBS). Caco-2 and HEK293T were from ATCC and cultured in DMEM (Gibco) supplemented with 10\% FBS. Calu-3 and Vero cells were from ATCC and cultured in EMEM (ATCC) supplemented with 15\% FBS. For the generation of primary bronchial epithelial cells, following attaining informed consent, airway segments and lung tissue were obtained from excess pathological tissue following lung transplantation in accordance with a protocol approved by the University of Pittsburgh Investigational Review Board (32). The isolation, growth and maintenance of these cells were as previously described $(31,32)$. Cells were treated with compound at indicated doses for indicated times, or at the following doses: HFG, $3 \mu \mathrm{M}$; Carfilzomib, $1 \mu \mathrm{M}$; Bafilomycin A1, $1 \mu \mathrm{M}$.

Cloning - HiBiT-tagged TMPRSS2 and SARS-2-CoV spike protein plasmid constructs were generated using molecular cloning and the FLEXI system (Promega). Briefly, the open reading frame of the target genes were PCR amplified with restriction sites for AsiSI and PmeI and was cloned into pFC37K-HiBiT plasmid. Point mutants (Lysine $\rightarrow$ Arginine) were generated through QuikChange II XL Site-Directed Mutagenesis Kit (Aglient). All plasmid constructs were verified by DNA sequencing (Genewiz). 
Transfection - Plasmid transfections were conducted using nucleofection in Beas-2b and MLE12 cells using Nucleofector II (Amaxa). X-tremeGENE HP DNA transfection reagent or Lipofectamine 3000 transfection reagent was used for plasmid transfections of HEK293T cells.

FDA-Approved Compound Library Screening - Human bronchial epithelial Beas-2b cells transiently expressing TMPRSS2-HiBiT (C-terminal) were seeded to a final density of $1 \times 10^{4}$ cells per well. The FDA-approved compound library (100nL per drug) was stamped to 384-well tissue culture plates using CyBio Well vario (Analytik Jena). Compounds were plated to the final concentrations of $10 \mu \mathrm{M}$. After $18 \mathrm{hr}$ of treatment, culture media was removed and cells were processed for Nanoluciferase activity using Extracellular HiBiT detection system (Promega), according to manufacturer's protocol. After reading the extracellular HiBiT signals, TritonX-100 solution was added into each well (final concentration 0.05\%) for cell lysis, and HiBiT signals were acquired again for all plates. Signals were collected and quantified using a Cytation 5 plate reader from Biotek. For secondary screening to determine drug IC50, specific compounds were cherry-picked using a TTP Mosquito X1 followed by serial dilutions of compounds were prepared using a Bravo automated liquid handling platform (Agilent). Cell seeding and extracellular and lytic HiBiT signal acquisition were performed according to the same protocol described above.

High-Throughput Liquid Handling - A Thermo Scientific custom HTS platform and Agilent Bravo automated liquid-handling platform was used to transfer contents of a FDA-approved compound library into assay plates. Biotek EL406 washer dispenser was used to distribute reagents or cell solutions into assay plates. For multiple plates operation, plate and liquid handling sequence and intervals were controlled through the Agilent VWORKs software. 
Cell Viability Assessment - Cell viability was tested using CellTiter-Glo 2.0 Cell Viability Assay (Promega). $20 \mu 1$ reagent was dispensed directly into each well of the 384-well tissue culture plates prior to luminescence signal acquisition by Cytation 5 plate reader.

Compound Washout Assays - Cells were pre-treated with indicated compound for 18-hr prior to 1 round of washing and incubation with fresh culture media for the indicated time periods. After the indicated times, cells were collected and processed for TMPRSS2 immunoblotting.

$R T-q P C R$ - Total RNA was extracted using RNA Extraction Miniprep Kit from Bioland Scientific, following the manufacturer's protocol. cDNA was prepared using High-Capacity RNAto-cDNA Kit from Applied Biosystems. SYBR Green Real-Time PCR Master Mixes from Applied Biosystems were used in qPCR, detecting the expression level TMPRSS2, HPN, ST14 and CORIN.

Ubiquitination Assay — TMPRSS2-V5-HIS in pcDNA3.1D was co-expressed with HA-DCAF1 in pcDNA3 in Beas-2B cells for 18hr, prior to lysis and precipitation with Dynabead HIS-resin (Thermo). Precipitate was eluted in 1xLaemmli Protein Sample Buffer at 95C for 10min, and resolved through SDS-PAGE immunoblotting.

Ubiquitination siRNA screen — TMPRSS2-HiBiT cells were screened with an siRNA library targeting Ubiquitination-related machinery as previously described (38). Briefly, 25 ng of siRNA was mixed with Lullaby transfection reagent (OzBiosciences) and diluted in Opti-MEM media. The transfection mixture incubated at room temperature for 20 minutes, and was added to $20 \mu \mathrm{L}$ of HITES $+10 \%$ FBS media with 2000 cells. Following $72 \mathrm{hr}$ knockdown, Lytic HiBiT luciferase assays were performed using manufacturer's protocol. For conformational specific gene silencing, small interfering RNAs were selected and purchased from IDT, and transfected in cells using 
Lullaby siRNA transfection reagent, with Negative Control DsiRNA transfected as control. Subsequent analysis was performed after $72 \mathrm{~h}$ of knockdown.

In vitro transcription and translation - TMPRSS2 constructs were synthesized in vitro using TnT Coupled Reticulocyte Lysate System (Promega) following manufacturer's protocol. HFG plus proline assays were conducted as previously described (27), with free amino acids diluted 5-fold and proline supplemented at indicated concentrations.

Immunoblotting - Cells were lysed in RIPA buffer supplemented with EDTA-free protease inhibitor tablet on ice. Cell lysates were sonicated at $20 \%$ amplification for 12 seconds and centrifuged at $12,000 \mathrm{~g}$ for 10 minutes at $4{ }^{\circ} \mathrm{C}$. Supernatants were collected and normalized for the total protein concentrations, mixed with $6 \mathrm{X}$ protein sample buffer, and incubated at $42^{\circ} \mathrm{C}$ for 10 minutes. Sample lysate was resolved using 4-20\% acrylamide PROTEAN ${ }^{\circledR}$ TGX $^{\mathrm{TM}}$ precast gels from BioRad and electrophoresed in TGS buffer. The proteins were then electro-transferred to nitrocellulose membranes. Blots were incubated in $15 \mathrm{ml}$ of blocking buffer for $1 \mathrm{hr}$ at room temperature, before incubation in $10 \mathrm{ml}$ of the primary antibody solution (1:2000 dilution) overnight at $4{ }^{\circ} \mathrm{C}$. Afterwards, three 10 -minute washing were performed in $15 \mathrm{ml}$ TBST. Blots were then incubated with $10 \mathrm{ml}$ of the secondary antibody solution for $1 \mathrm{hr}$ at room temperature. After three 10-minute washing in 15ml TBST, blots were then developed using West Femto Maximum Sensitivity Substrate from Thermo Scientific, and imaged using ChemiDoc Imaging System from Bio-Rad.

HiBiT Blotting - Samples from cells transfected with HiBiT-tagged proteins were prepared following the same protocol as immunoblotting. Proteins were transferred to a nitrocellulose membrane, followed by gentle rocking in TBST to rinse away transfer buffer. Nano-Glo HiBiT blotting system (Promega) was used for development, following manufacturer's protocol. Briefly, 
the blot was incubated in $5 \mathrm{ml} 1 \times$ Nano-Glo blotting buffer supplemented with $25 \mu \mathrm{LgBiT}$ protein overnight at $4{ }^{\circ} \mathrm{C}$. The next day, $10 \mu \mathrm{l} \mathrm{Nano-Glo} \mathrm{luciferase}$ assay substrate was directly added into the solution and mixed well immediately. After incubation for $5 \mathrm{~min}$ at room temperature in dark, the blot was imaged by ChemiDoc Imaging System (Bio-Rad), using chemiluminescence mode.

Pseudovirus Entry Assays - Pseudovirus with SARS-CoV-2 spike protein with a C-terminal HiBiT tag was generated by co-transfection of 293T cells with psPAX2 (Addgene, MA), pLentic-mGFP (Origene, MD) and PFC37K-HIBIT-SARS-CoV-2-S (Backbone was from Promega, WI) by using lipofectamine 3000 (Invitrogen, CA). Briefly, 293T cells were seeded one day before in $8 \mathrm{ml}$ DMEM complete media without antibiotics in a $10 \mathrm{~cm}$ tissue culture dish. The following morning, cells were transfected with $8 \mu \mathrm{g}$ psPAX2, $8 \mu \mathrm{g}$ pLenti-c-mGFP or pLenti-c-FLUC and 4 $\mu \mathrm{g}$ PFC37K-HIBIT-SARS-CoV-2-S with lipofectamine 3000 according to the manufacturer's protocol. Six hours later, media was changed with fresh full media without antibiotics. The supernatants were harvested $48 \mathrm{~h}$ post-transfection, and centrifuged at $500 \times \mathrm{g}$ for $10 \mathrm{~min}$ to remove cell debris. Virus titer was checked by Lenti-X GoStix Plus (Takara,CA). HibiT expression was checked by Nano-Glo® HiBiT Lytic Detection System (Promega, WI). The HIV-1 Gag p24 content in the produced SARS-CoV-2-HiBiT pseudovirus was quantified by ELISA (R\&D systems), following the manufacturer's protocol. To detect infectivity, various cell types with control or compound treatment were incubated for $1 \mathrm{~h}$ with pseudovirus, after extensive washes with PBS, cells were lysed with Nano-Glo HiBiT lytic reagent (Promega) for $20 \mathrm{~min}$. Luminescence signals were then acquired by ClarioSTAR microplate reader (BMG Labtech, Cary, NC).

Immunocytochemistry - Caco-2 cells were seeded in 384-well glass-bottom plates (Cellvis, 5000 cells/well) and treated with halofuginone or homoharringtonine at the indicated concentrations for 
18hr. Cells were then treated with a pseudovirus encoding the SARS-CoV-2 Spike protein and mGFP. Forty-eight hours after infection, cells were fixed (4\% paraformaldehyde), permeabilized (0.5\% Triton X-100), and stained for GFP (Cell Signaling Technology), followed with a goat antirabbit Alexa Fluor 568 secondary antibody. The fluorescent signal was imaged using Image Express (Molecular Devices) to measure viral entry.

TMPRSS2 activity assay — Test compounds were pre-incubated with recombinant TMPRSS2 (AA 106-492, LSBio) in assay buffer (TBS, 0.05\% Tween-20) for 15 minutes before addition of BocGln-Ala-Arg-MCA (Peptides International) substrate to a final concentration of $10 \mu \mathrm{M}$. Final concentration of TMPRSS2 was $1.35 \mu \mathrm{g} / \mathrm{mL}$. Fluorescence (380-15nm excitation/470-20nm emission) of the samples was monitored over 4:45 hours and the slope of the curves was calculated by linear regression analysis and used as output for TMPRSS2 activity.

Cellular thermal shift assay — B2B cells were transfected with TMPRSS2-HiBiT plasmid overnight, prior to treatment with vehicle or HFG ( $3 \mu \mathrm{M}$ for $1 \mathrm{hr})$. Cells were collected and resuspended in $10 \mathrm{ml}$ of PBS supplemented with EDTA-free protease inhibitor tablet. Cell solutions were aliquoted to 10 PCR microtubes evenly. Using the PCR thermocycler generating temperature gradient, each aliquot was incubated at a certain temperature between $40 \sim 58^{\circ} \mathrm{C}$ with $2^{\circ} \mathrm{C}$ interval for $3 \mathrm{~min}$, then at room temperature for $3 \mathrm{~min}$. Samples were immediately snap frozen in liquid nitrogen and 2 cycles of freeze-thaw were followed. After vortex briefly, samples were transferred to $1.7 \mathrm{ml}$ microcentrifuge tubes for centrifuging at $20,000 \mathrm{~g}, 4^{\circ} \mathrm{C}$ for $20 \mathrm{~min}$. Supernatants were carefully acquired and used for subsequent immunoblotting analysis. The whole procedure followed the protocol described in previous literature (51). 
Statistics - Statistical comparisons were performed in GraphPad Prism. Unpaired two-tailed Student's $t$-test was used to compare two groups. Comparisons of more than two groups were tested with one-way ANOVA with Tukey's post-hoc test of multiple comparisons.

\section{Supplemental Figure Legends:}

Supplementary Figure 1: Assessment of TMPRSS2 HTS Screen. TMPRSS2-HiBiT expressing BEAS-2B cells were seeded into 384-well plates for 24h. Half of the cells were treated with CHX overnight and the lytic HiBiT signal was measured. A subsequent Z'-factor was calculated.

Supplementary Figure 2: IC50 calculations for compounds that reduce TMPRSS2 expression. Remaining compounds (top 24 in total) inhibiting TMPRSS2 expressions were tested for their potency in reducing extracellular (first column) or total (middle column) TMPRSS2-HiBiT protein levels. An assessment of cellular toxicity for each compound (CellTiter-Glo) is shown in the last column.

Supplementary Figure 3: Agents identified in the screen selectively decrease TMPRSS2 in airway cells. TMPRSS2-HiBiT expressing MLE-12 cells were treated with three agents identified in the screen (homoharringtonine, halofuginione, or venetoclax) at the indicated concentration for $18 \mathrm{hr}$ prior to chemiluminescent detection of TMPRSS2-HiBiT expression. Conventional detection of actin is shown as a loading control.

Supplementary Figure 4: Analysis of TMPRSS2 mRNA expression by qPCR analysis of Caco2 cells treated with homoharringtonine or halofuginone for $6 \mathrm{hr}$. Expression of other related proteases including hepsin (HPN), ST14, and corin were also measured. Data represent mean $-/+$ SEM $(\mathrm{n}=4) .{ }^{*} \mathrm{P}<0.05$, relative to vehicle, or as indicated by one-way ANOVA with Dunnett's test of multiple comparisons.

Supplementary Figure 5: (A-B) Calu-3 cells were treated with a dose course of homoharringtonine (HHT) or halofuginone (HFG) for $18 \mathrm{hr}$ prior to immunoblotting analysis. Densitometry of endogenous TMPRSS2 is shown below. Data represent mean $-/+$ SEM $(n=3)$. (C) Time-course treatment of HHT-treated Caco-2 cells $(3 \mu \mathrm{M})$. TMPRSS2 densitometry is shown, data are mean +/- SEM (n=3). (D) Immunoblot analysis of Caco-2 cells treated with HHT for $18 \mathrm{hr}$ prior to removing the drug, adding fresh media, and then analyzing the protein recovery time course. 
Supplementary Figure 6: (A) in vitro transcription/translation assay. V5- or HiBiT-tagged TMPRSS2 was treated with HFG without or with L-proline supplementation $(\mathrm{n}=3)$. (B) HFGinduced decrease in TMPRSS-HiBiT signal is blunted with exogenous proline addition to Beas2B cells. Data represent mean $+/-$ SEM $(n=8)$.

Supplementary Figure 7: (A-B) SARS-CoV-2 pseudovirus entry assay in Calu-3 cells following HHT or HFG treatment, corrected for cell viability with Celltiter-Glo and normalized to vehicle treatment. (C) SARS-CoV-2 pseudovirus entry assay in Caco-2 cells treated with calcium channel blocker cilnidipine. (D) Treatment of Caco-2 cells with the indicated agents. All agents were assessed at $10 \mu \mathrm{M}$ except for camostat $(3 \mu \mathrm{M})$. data are mean $-/+$ SEM $n=3$, SARS-CoV-2 pseudoviral luminescence is corrected for cell viability with CellTiter-Glo and normalized to vehicle treatment. $* \mathrm{P}<0.05$, relative to 0 time point or control, or as indicated by one-way ANOVA with Dunnett's test of multiple comparisons (A-B).

Supplementary Figure 8: (A-B) Effects of HHT or HFG on pseudoviral-mediated FLUC expression in Caco2 cells. data are mean -/+ SEM ( $n=4)$., SARS-CoV-2 pseudoviral FLUC is corrected for cellular protein concentration and normalized to vehicle treatment. (C) Pseudoviral entry assay with HHT $(10 \mathrm{nM})$, HRG $(10 \mathrm{nM})$ alone or combined, data are mean $-/+$ SEM $(\mathrm{n}=3)$. $* \mathrm{P}<0.05$, relative to 0 time point or control, or as indicated by one-way ANOVA with Dunnett's test of multiple comparisons (A-C).

Supplementary Figure 09: in vitro fluorescent activity assay of recombinant TMPRSS2 in the presence of a dose course of several compounds $(\mathrm{N}=4)$

Supplementary Figure 10: Cellular thermal shift assay of TMPRSS2 (A) and DCAF1 (B) proteins in B2B cells transfected with TMPRSS2-HiBiT plasmid, upon treatment with vehicle or HFG. The abundance of TMPRSS2 and DCAF1 were detected by HiBiT blotting and antiDCAF1 antibody respectively. The corresponding quantifications of densitometry were shown beneath.

Supplementary Figure 11: Strategies to limit SARS-CoV-2 infection. Agents identified here act by reducing the expression of TMPRSS2 and thereby limiting viral entry. Combinations of these agents may synergize which each other to reduce TMPRSS2 levels below what a single agent can achieve. In addition, agents that reduce TMPRSS2 levels or function would also likely synergize with agents (e.g. chloroquine) that predominantly target viral entry through the endosomal pathway. 
Fig.1

A

HiBiT-Nanoluciferase System

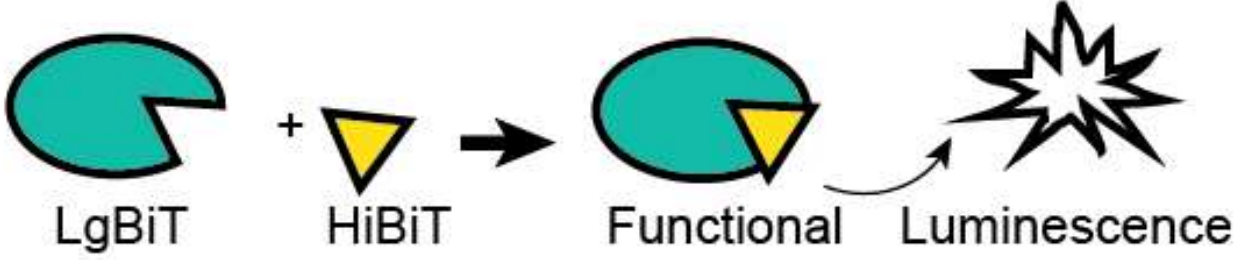

Fragment Fragment Nano-Luciferase

$\mathrm{B}$

Tagging of Essential Protease for SARS-CoV-2 Infectivity

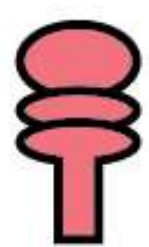

TMPRSS2

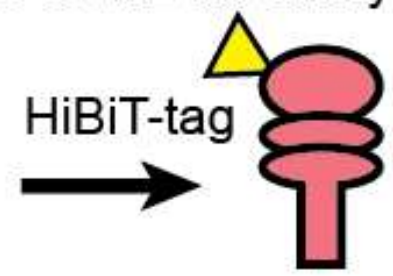

TMPRSS2
C

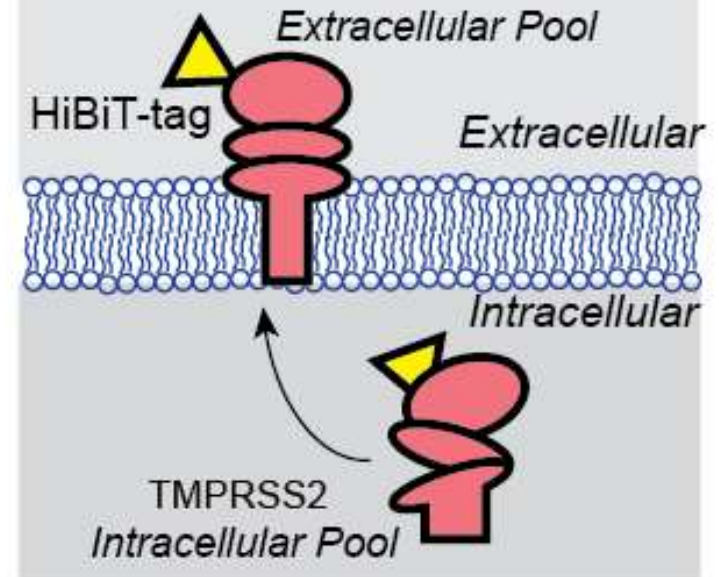

D TMPRSS2-HiBiT Expression in Airway Epithelial Cells

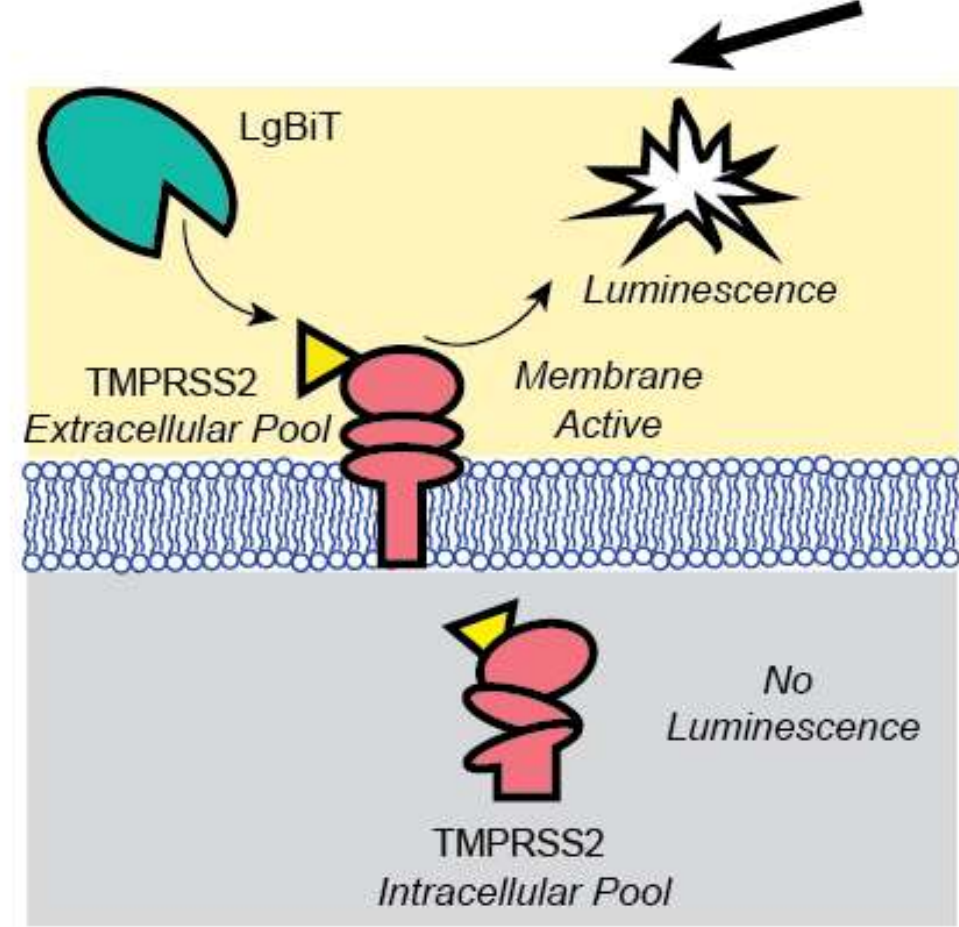

Extracellular HiBiT Detection

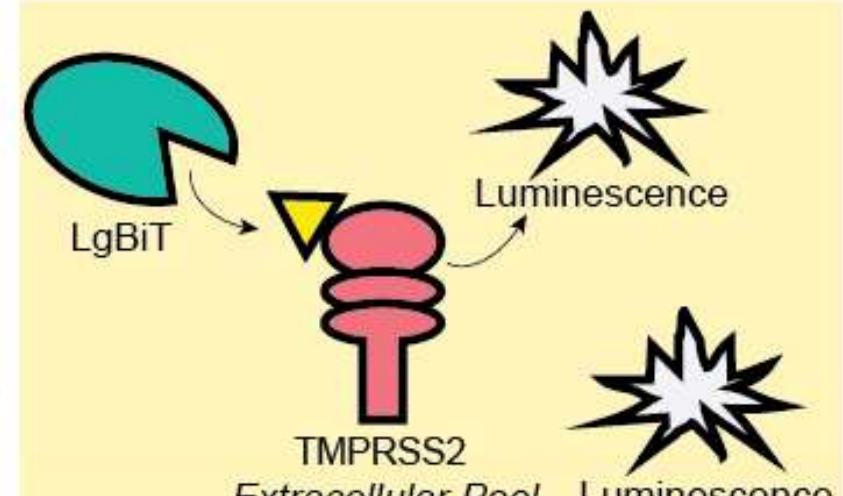

Extracellular Pool Luminescence

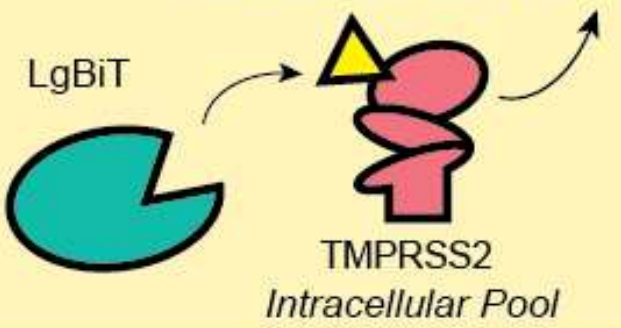

Lytic HiBiT Detection 
Fig. 2
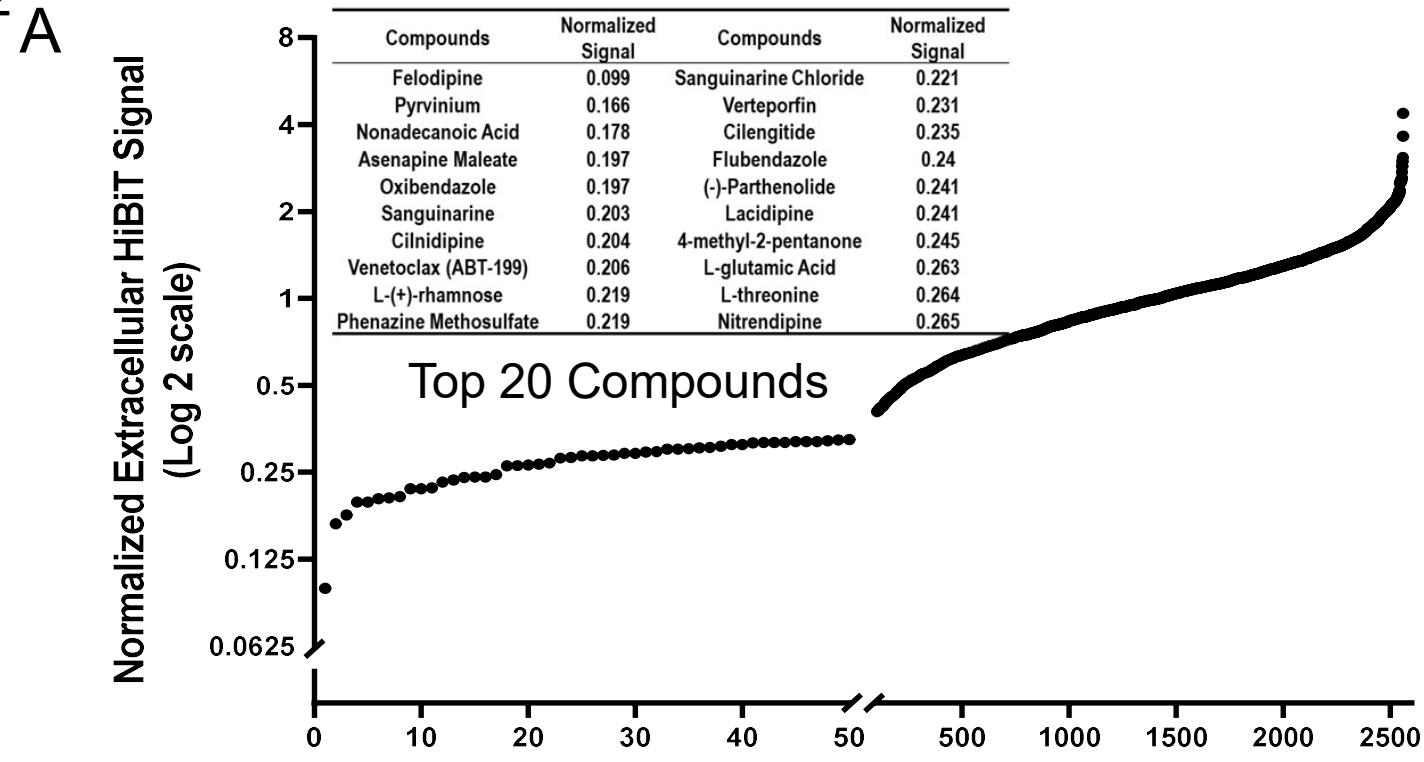

B

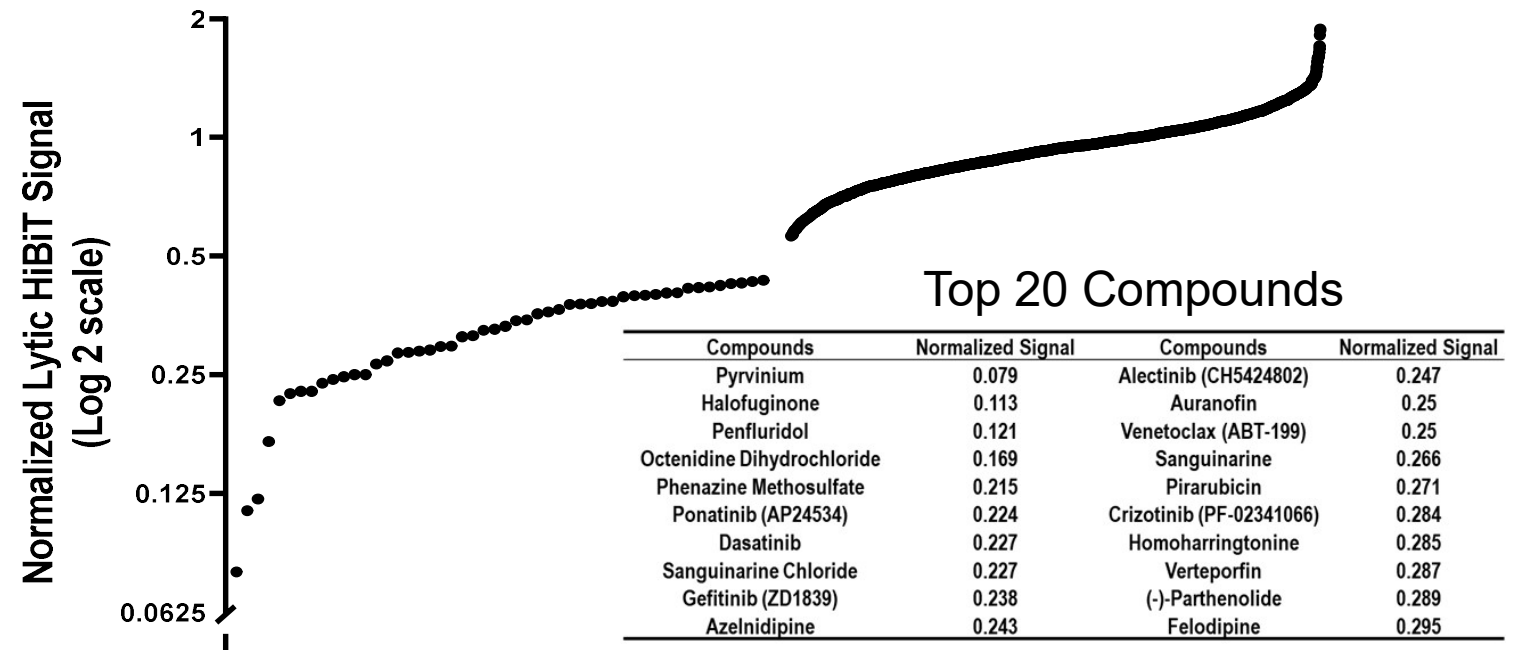

C

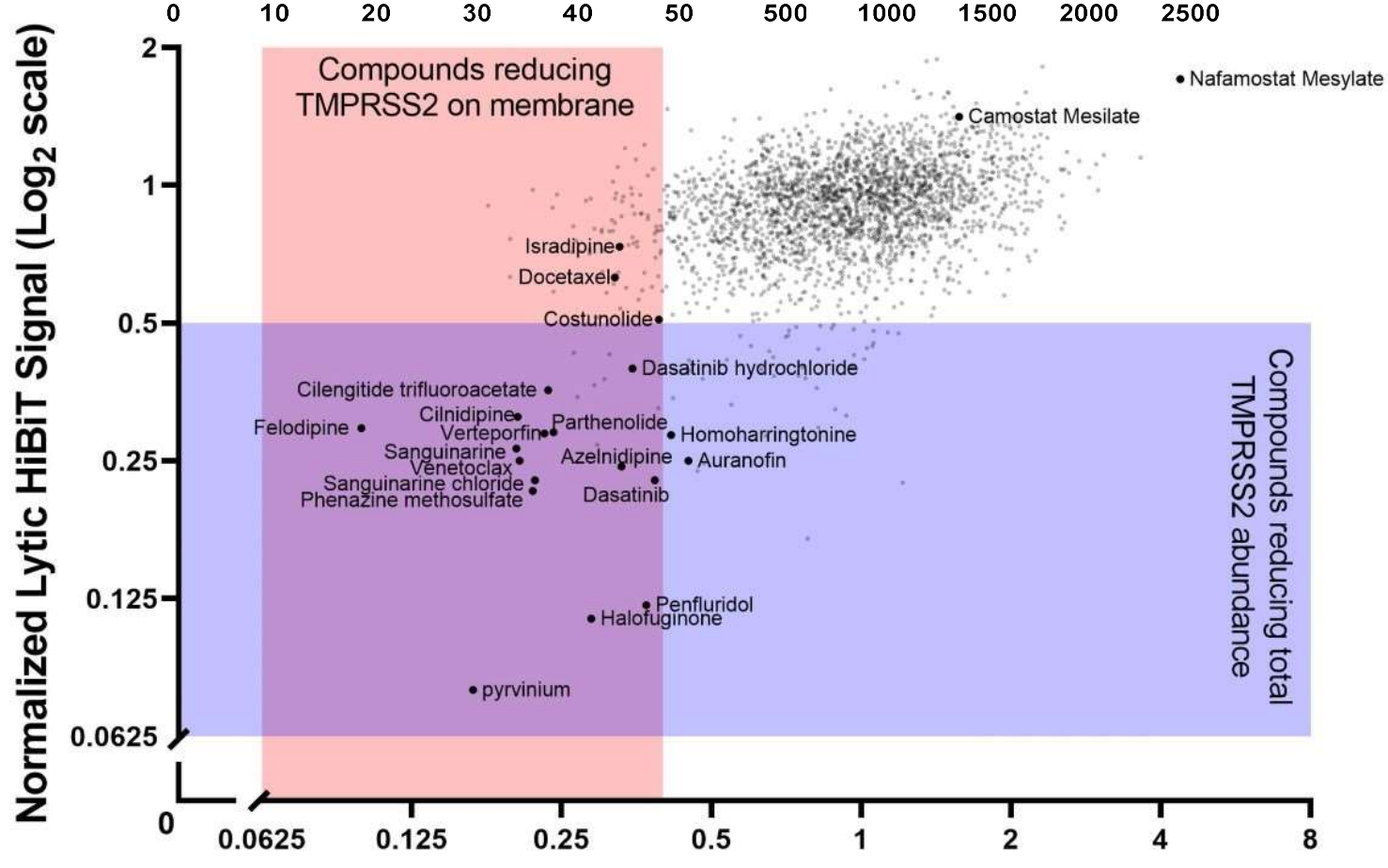

Normalized Extracellular HiBiT Signal ( $\log _{2}$ scale) 


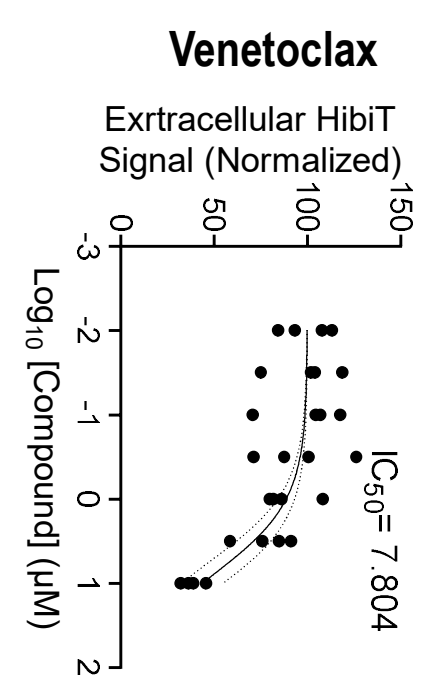

Lytic HiBiT Signal (Normalized)

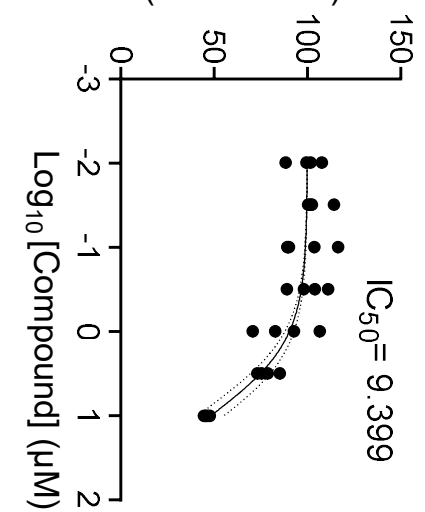

ATP-Glo (Normalized)

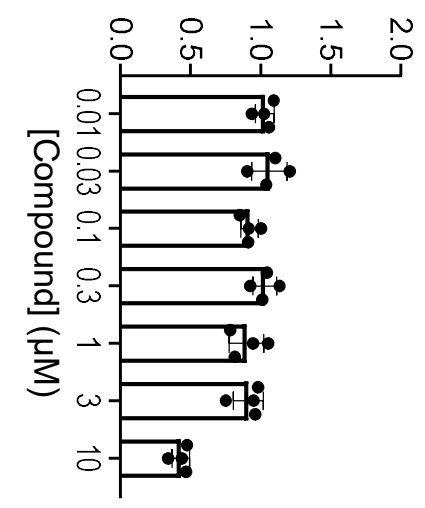

\section{Dasatinib}

Exrtracellular HibiT Signal (Normalized)

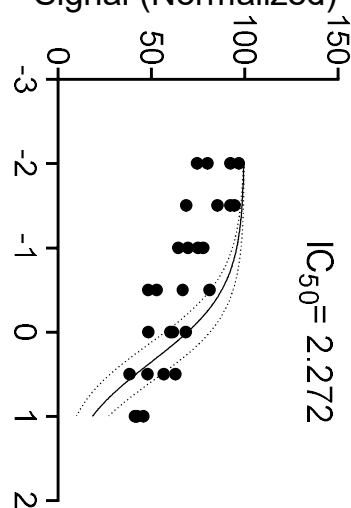

Lytic HiBiT Signal (Normalized)

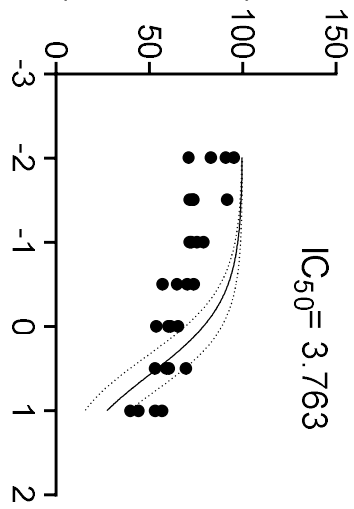

ATP-Glo (Normalized)

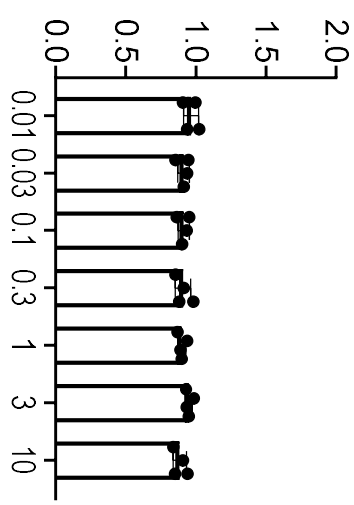

Verteporfin

Exrtracellular HibiT

Signal (Normalized)

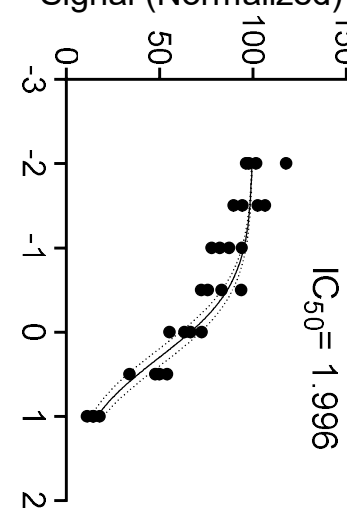

Lytic HiBiT Signal (Normalized)

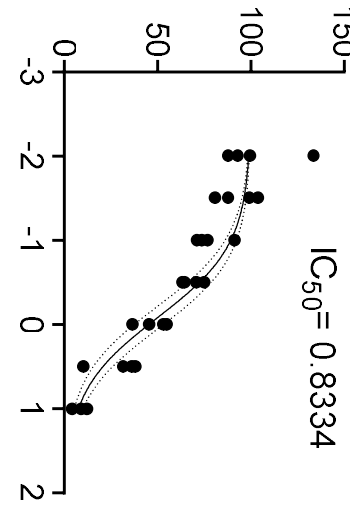

ATP-Glo (Normalized)

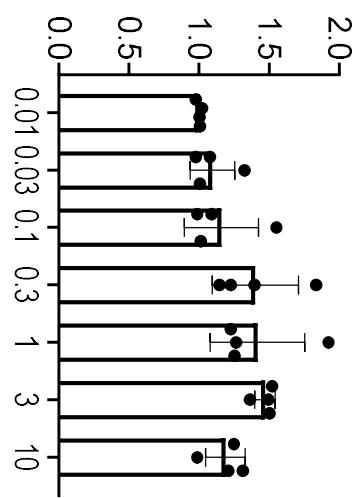

\section{Cilnidipine}

Exrtracellular HibiT

Signal (Normalized)

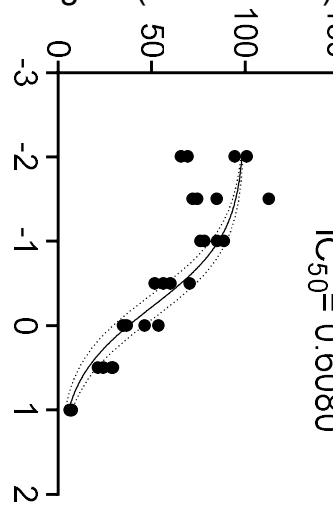

Lytic HiBiT Signal (Normalized)

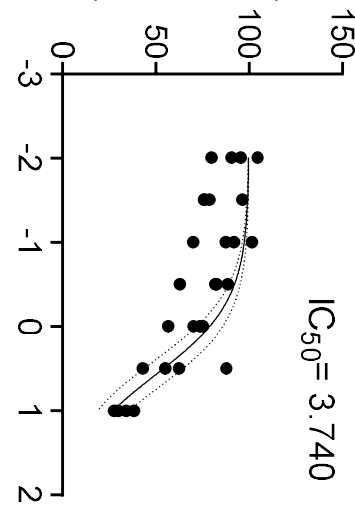

ATP-Glo (Normalized)

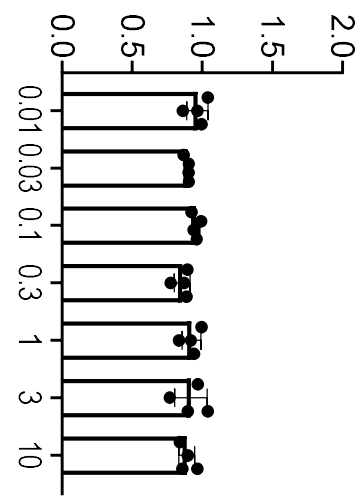

Halofuginone

Exrtracellular HibiT Signal (Normalized)

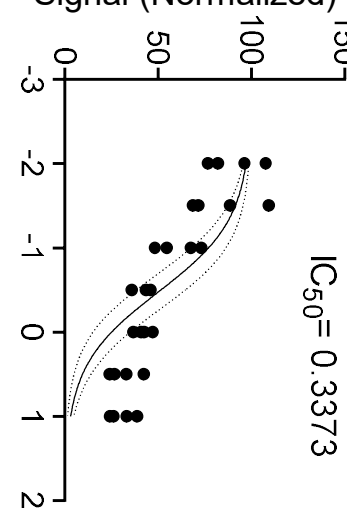

Lytic HiBiT Signal (Normalized)

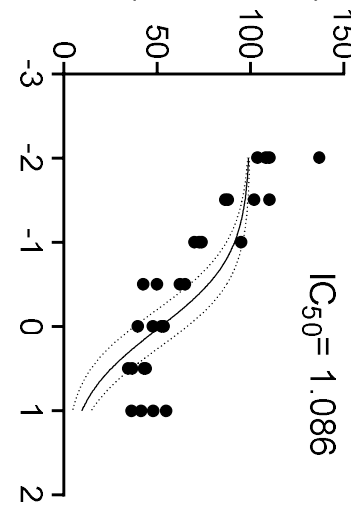

ATP-Glo (Normalized)

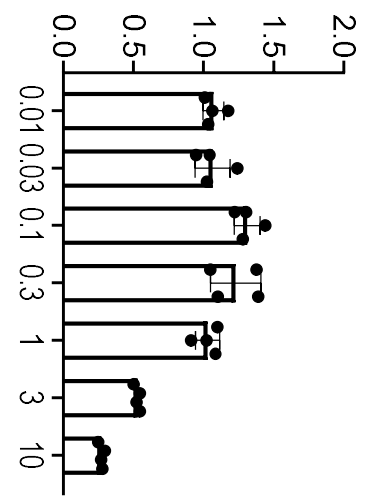

Homoharringtonine $\frac{7}{\sigma 9}$.

Exrtracellular HibiT $\omega$

Signal (Normalized)

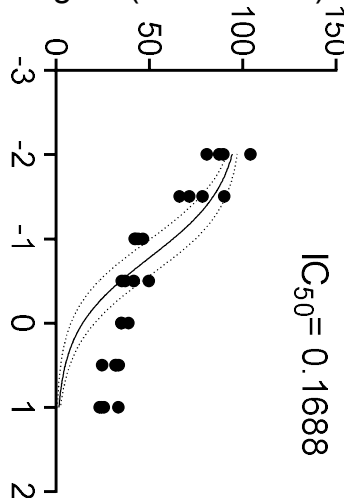

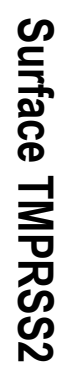

Lytic HiBiT Signal (Normalized)

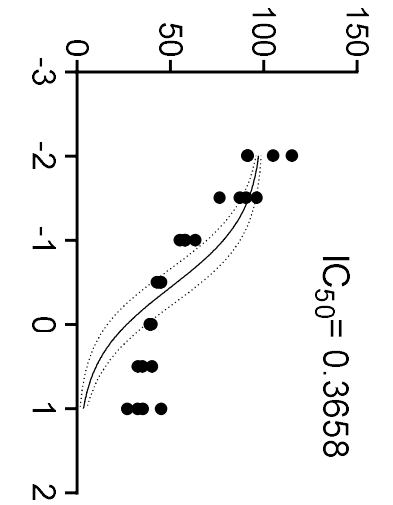

ATP-Glo (Normalized)

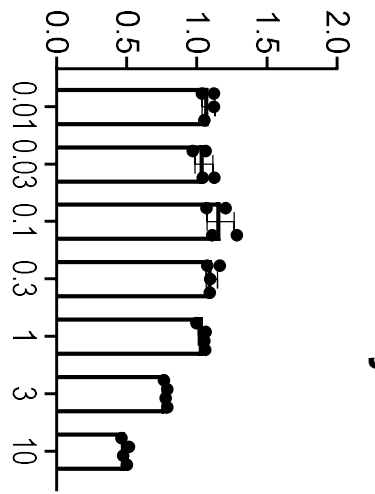


Fig.4
A
B
\begin{tabular}{llll}
\multicolumn{4}{c}{$\mathrm{HHT}$} \\
\hline 0 & 0.3 & 1 & 3
\end{tabular}
\begin{tabular}{lllll}
\multicolumn{3}{c}{$\mathrm{HFG}$} & \\
\hline 0 & 1 & 3 & 10 & $(\mu \mathrm{M})$
\end{tabular}
HHT HFG MLE-12 cells
Q
TMPRSS2
$\begin{array}{lllllllll}0 & 1 & 3 & 10 & 0 & 1 & 3 & 10 & (\mu \mathrm{M})\end{array}$
(0)

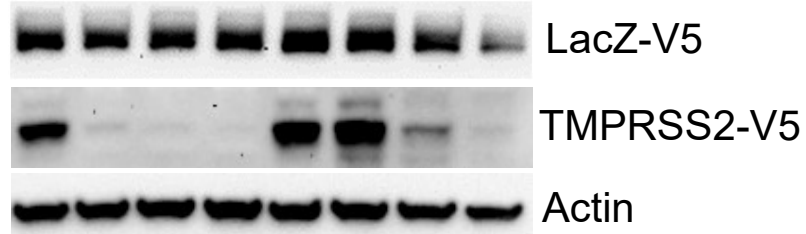

D
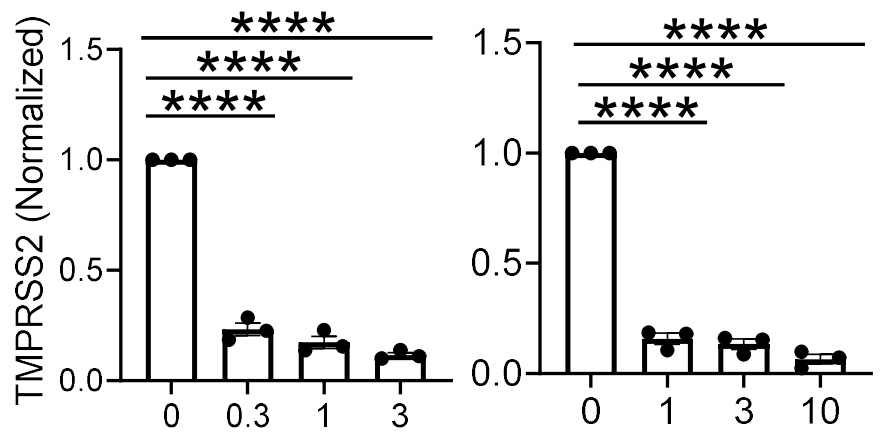

Homoharringtonine $(\mu \mathrm{M}) \quad$ Halofuginone $(\mu \mathrm{M})$

E

$\begin{array}{llll}0 & 3 & 6 & 24 \mathrm{~h}\end{array}$

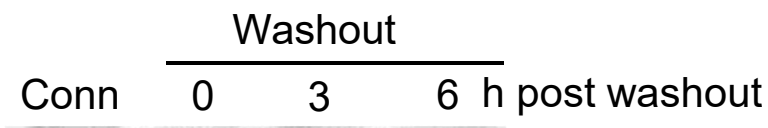

TMPRSS2

m $m$ m TMPRSS2

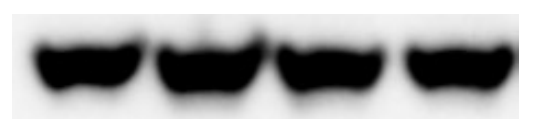

Actin
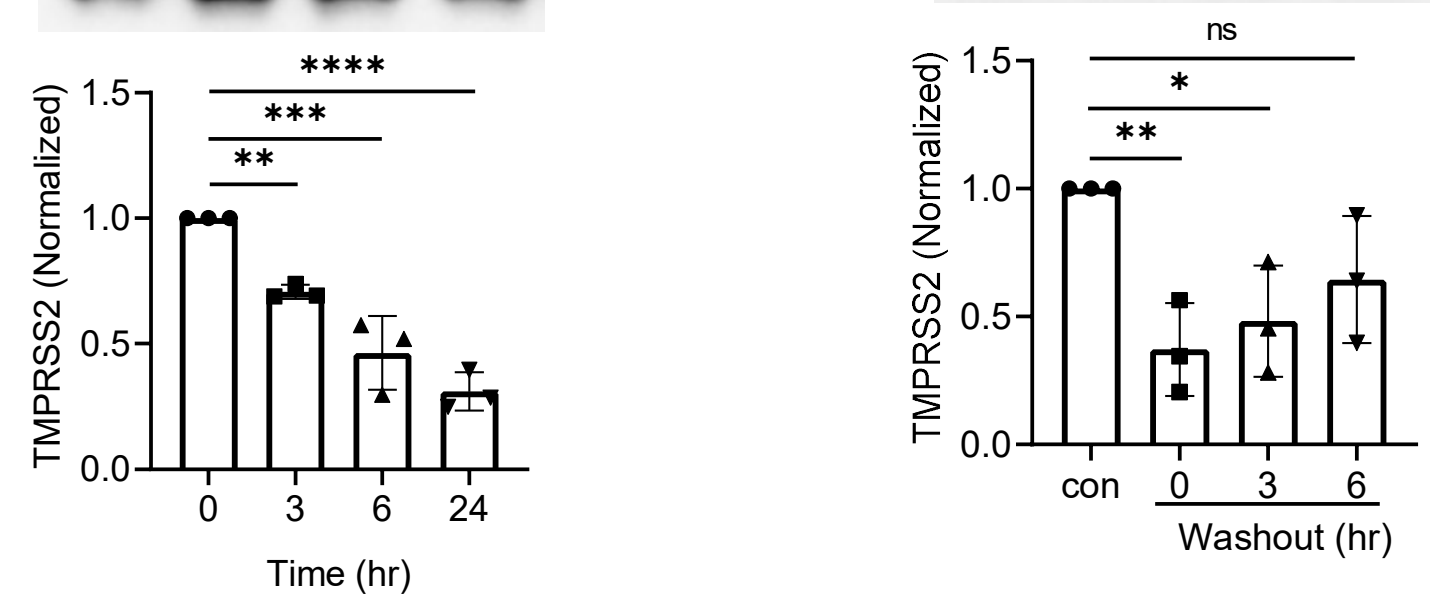

Actin 

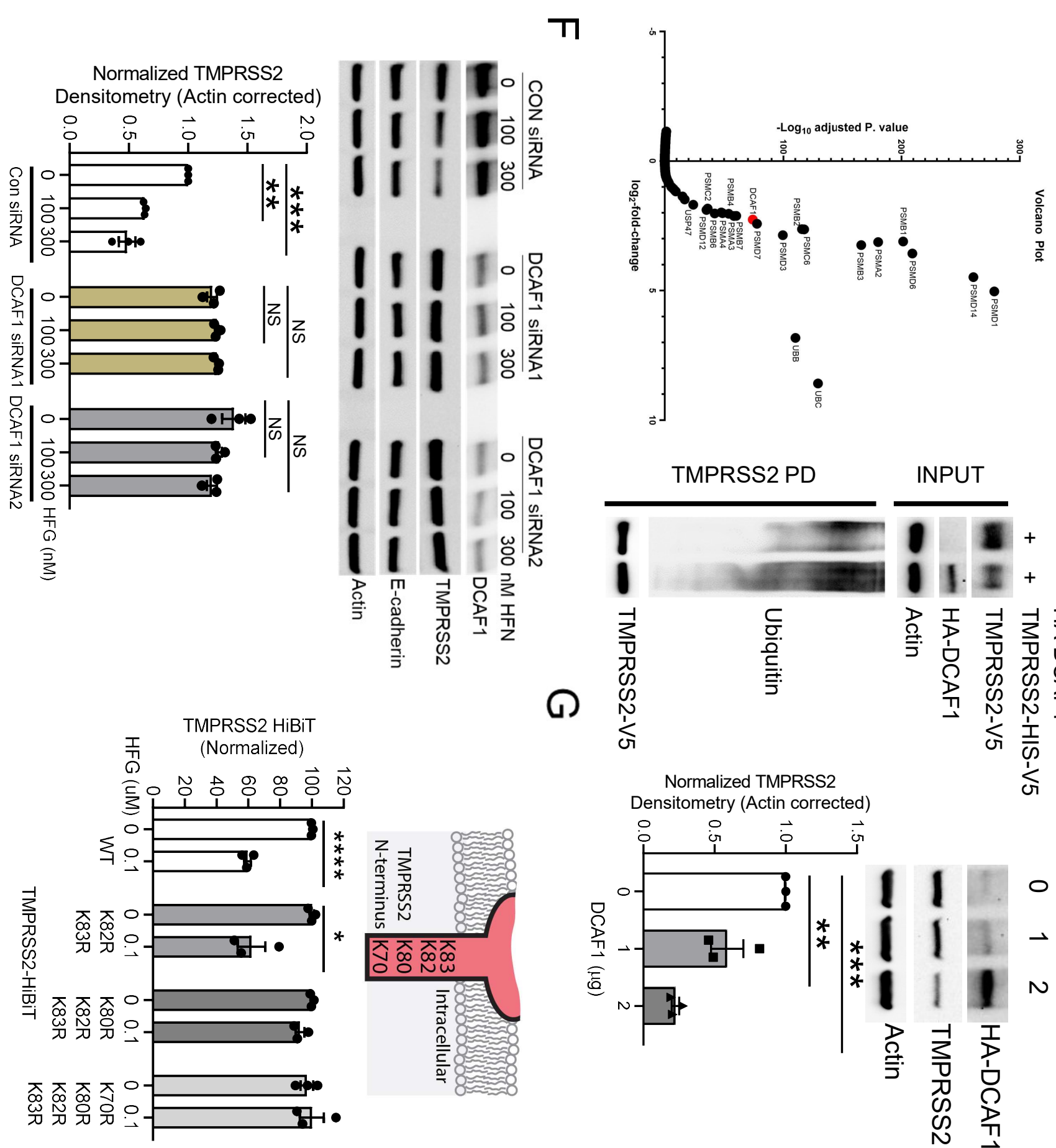

ก
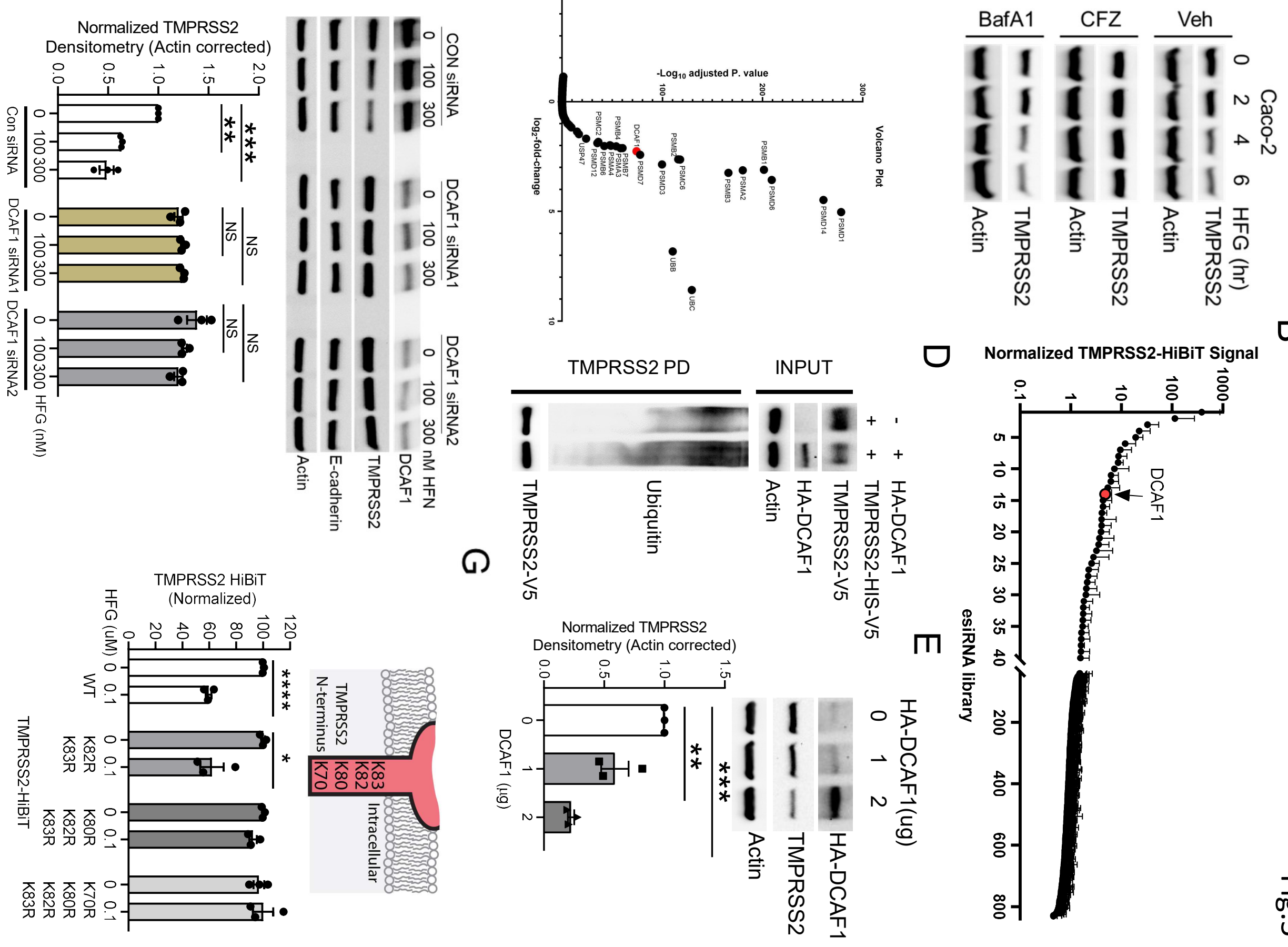

Normalized TMPRSS2-HiBiT Signal

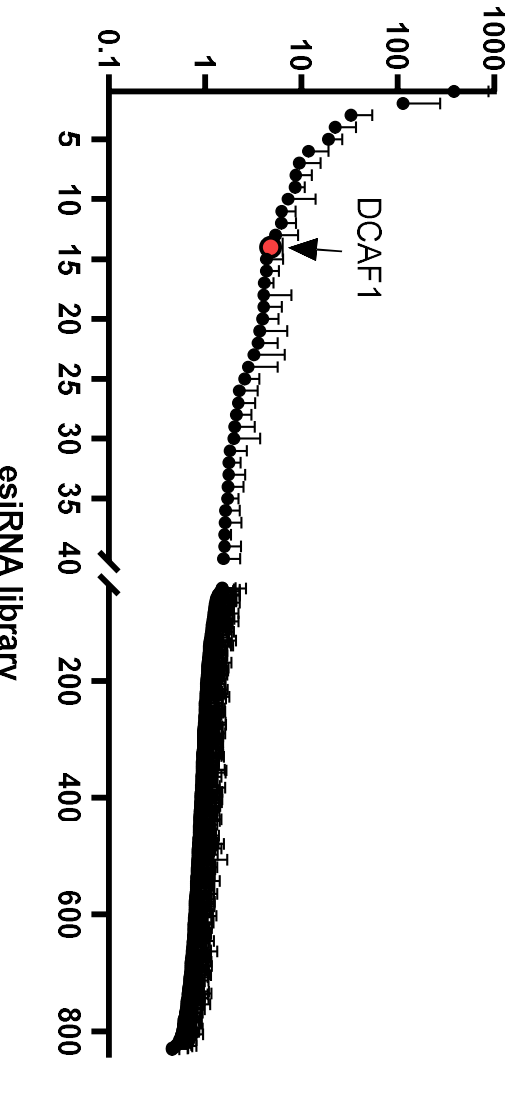


Fig.6

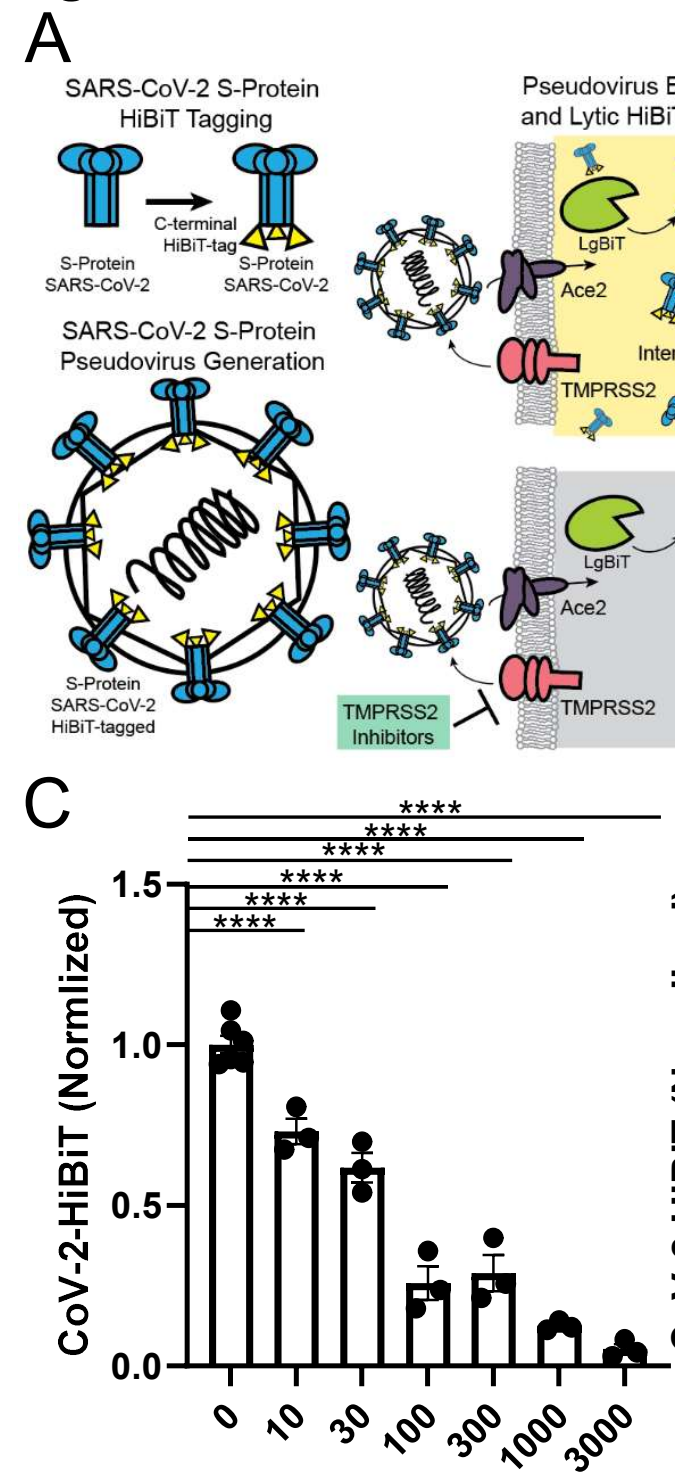

Homoharringtonine (nM)

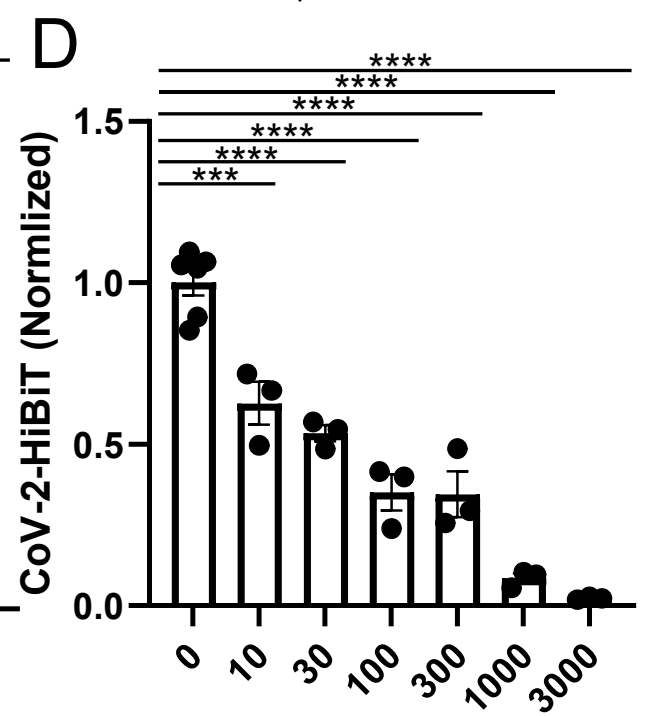

Halofuginone (nM)

F
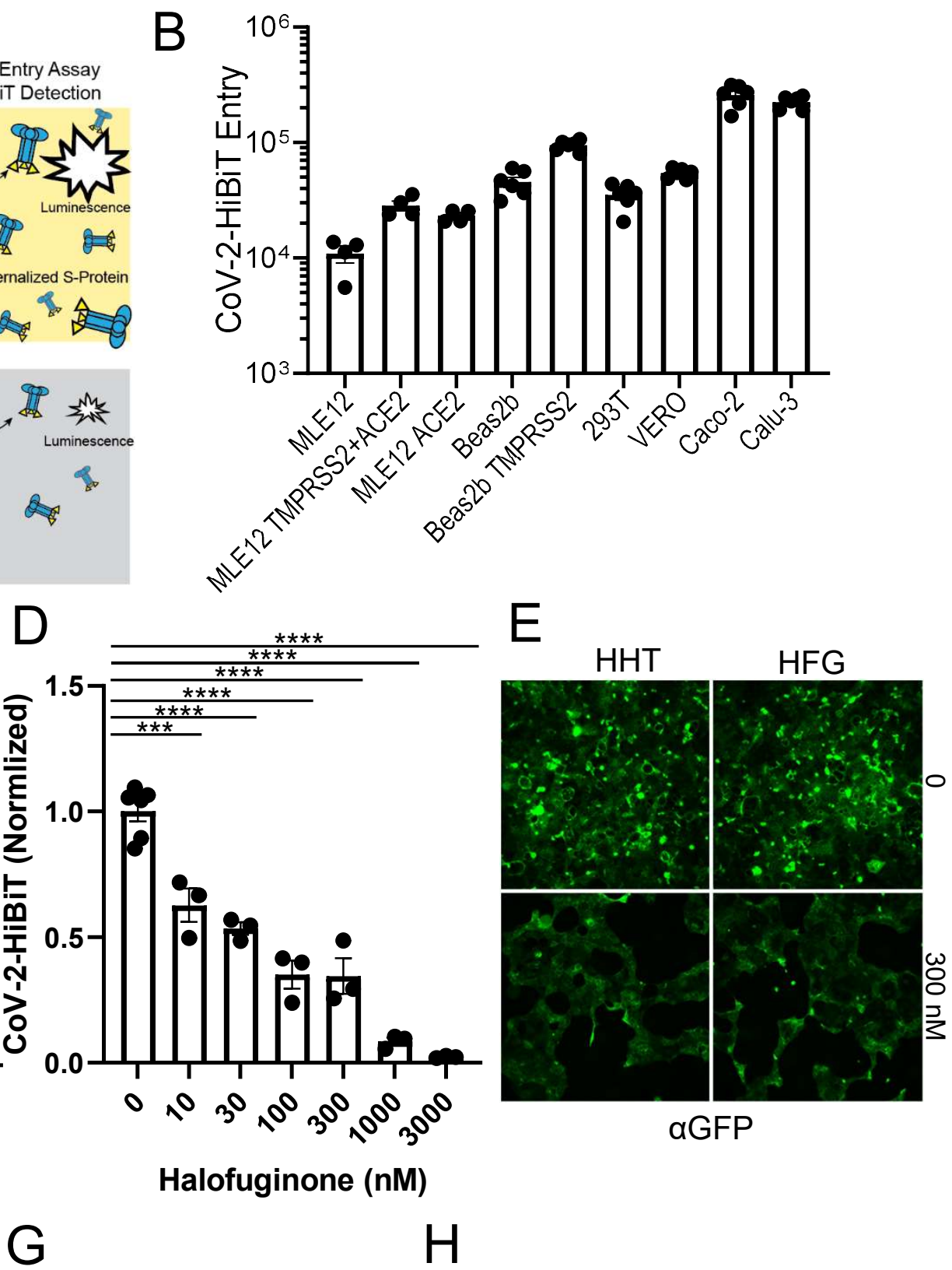

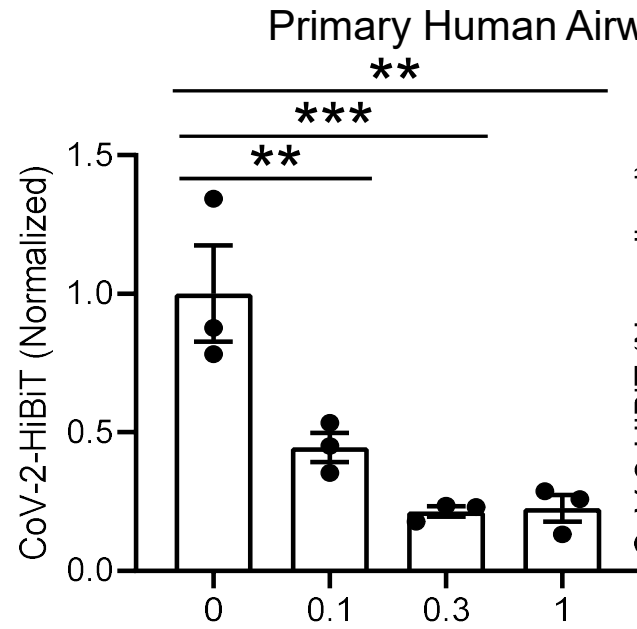

Homoharringtonine $(\mu \mathrm{M})$

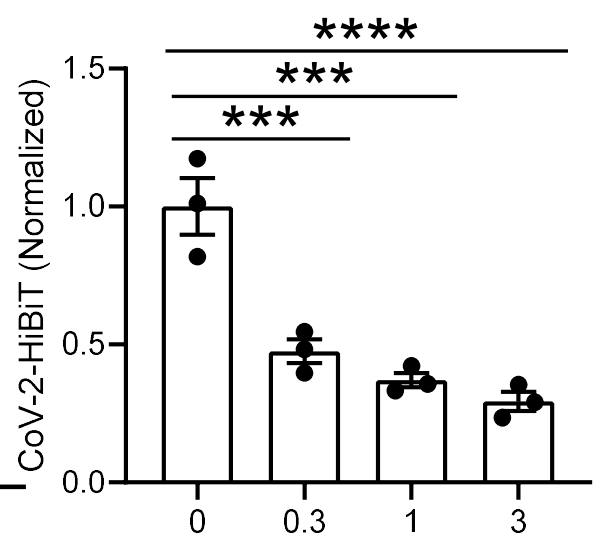

Halofuginone $(\mu \mathrm{M})$

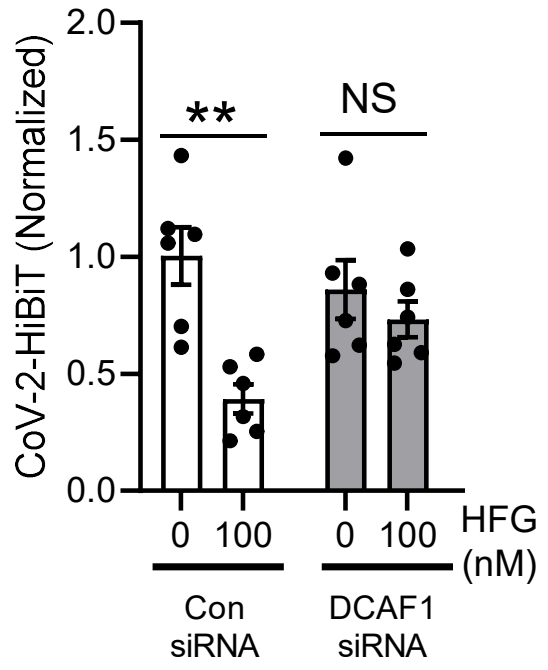


Table 1

\begin{tabular}{|c|c|c|c|c|c|c|c|}
\hline Drug & Disease & $\begin{array}{c}\text { Regulator } \\
\text { y Status }\end{array}$ & Dose & $\begin{array}{c}\text { Route } \\
\text { (frequen } \\
\text { cy) }\end{array}$ & Cmax & $\begin{array}{l}\text { In vitro } \\
\text { IC50 } \\
\text { (viral } \\
\text { entry) }\end{array}$ & $\begin{array}{c}\text { Referenc } \\
\text { e }\end{array}$ \\
\hline $\begin{array}{c}\text { Homoharringto } \\
\text { nine } \\
\text { (Omacetaxine) }\end{array}$ & $\begin{array}{l}\text { chronic } \\
\text { myeloid } \\
\text { leukemia } \\
(\mathrm{CML})\end{array}$ & $\begin{array}{c}\text { FDA } \\
\text { approved }\end{array}$ & $\begin{array}{c}1.25 \\
\mathrm{mg} / \mathrm{m} 2\end{array}$ & SC (BID) & 55nM & -30nM & [32] \\
\hline Halofuginone & scleroderma & Phase 1/2 & $3.5 \mathrm{mg} / \mathrm{day}$ & Oral & $7 \mathrm{nM}$ & $\sim 30 \mathrm{nM}$ & [33] \\
\hline Verteporfin & $\begin{array}{c}\text { photosensitizer } \\
\text { for } \\
\text { photodynamic } \\
\text { therapy }\end{array}$ & $\begin{array}{c}\text { FDA } \\
\text { approved }\end{array}$ & $0.3 \mathrm{mg} / \mathrm{kg}$ & $\begin{array}{l}\text { IV (within } \\
\text { 45min) }\end{array}$ & $1.92 \mu \mathrm{M}$ & $\sim 10 \mu \mathrm{M}$ & FDA \\
\hline Cilnidipine & Hypertension & $\begin{array}{c}\text { FDA } \\
\text { approved }\end{array}$ & $10 \mathrm{mg}$ & $\begin{array}{l}\text { Oral } \\
\text { (QD) }\end{array}$ & 18.1nM & $\sim 3 \mu \mathrm{M}$ & [34] \\
\hline Dasatinib & $\begin{array}{c}\text { chronic } \\
\text { myelogenous } \\
\text { leukemia } \\
\text { (CML) and } \\
\text { acute } \\
\text { lymphoblastic } \\
\text { leukemia (ALL) }\end{array}$ & $\begin{array}{c}\text { FDA } \\
\text { approved }\end{array}$ & $140 \mathrm{mg}$ & $\begin{array}{l}\text { Oral } \\
\text { (QD) }\end{array}$ & $0.307 \mu \mathrm{M}$ & $>10 \mu \mathrm{M}$ & [35] \\
\hline Venetoclax & $\begin{array}{c}\text { chronic } \\
\text { lymphocytic } \\
\text { leukemia (CLL } \\
\text { ) or small } \\
\text { lymphocytic } \\
\text { lymphoma (SL } \\
\text { L) }\end{array}$ & $\begin{array}{c}\text { FDA } \\
\text { approved }\end{array}$ & $400 \mathrm{mg}$ & $\begin{array}{l}\text { Oral } \\
\text { (QD) }\end{array}$ & $1.27 \mu \mathrm{M}$ & $>10 \mu \mathrm{M}$ & [36] \\
\hline
\end{tabular}


Fig. S1

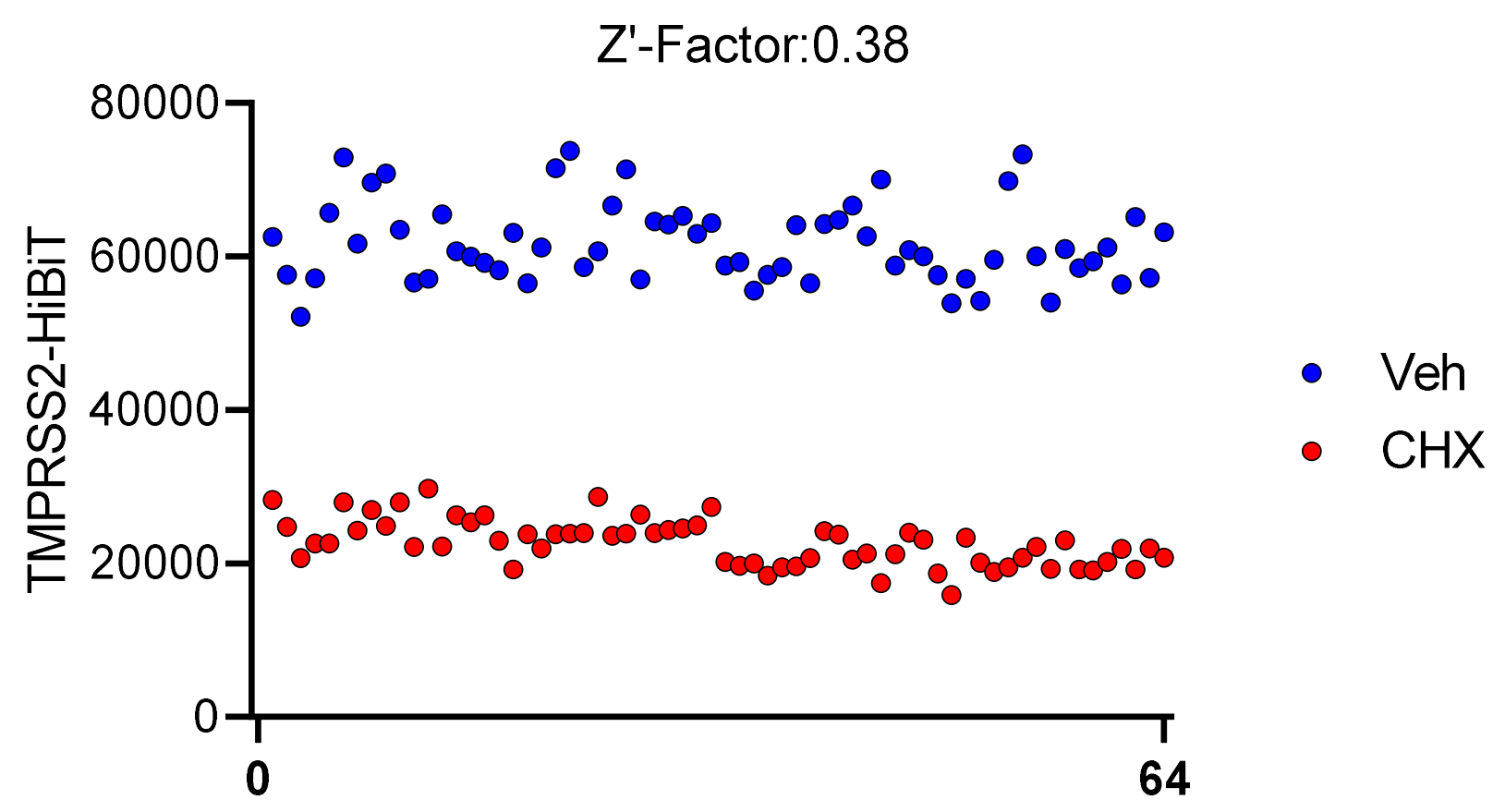




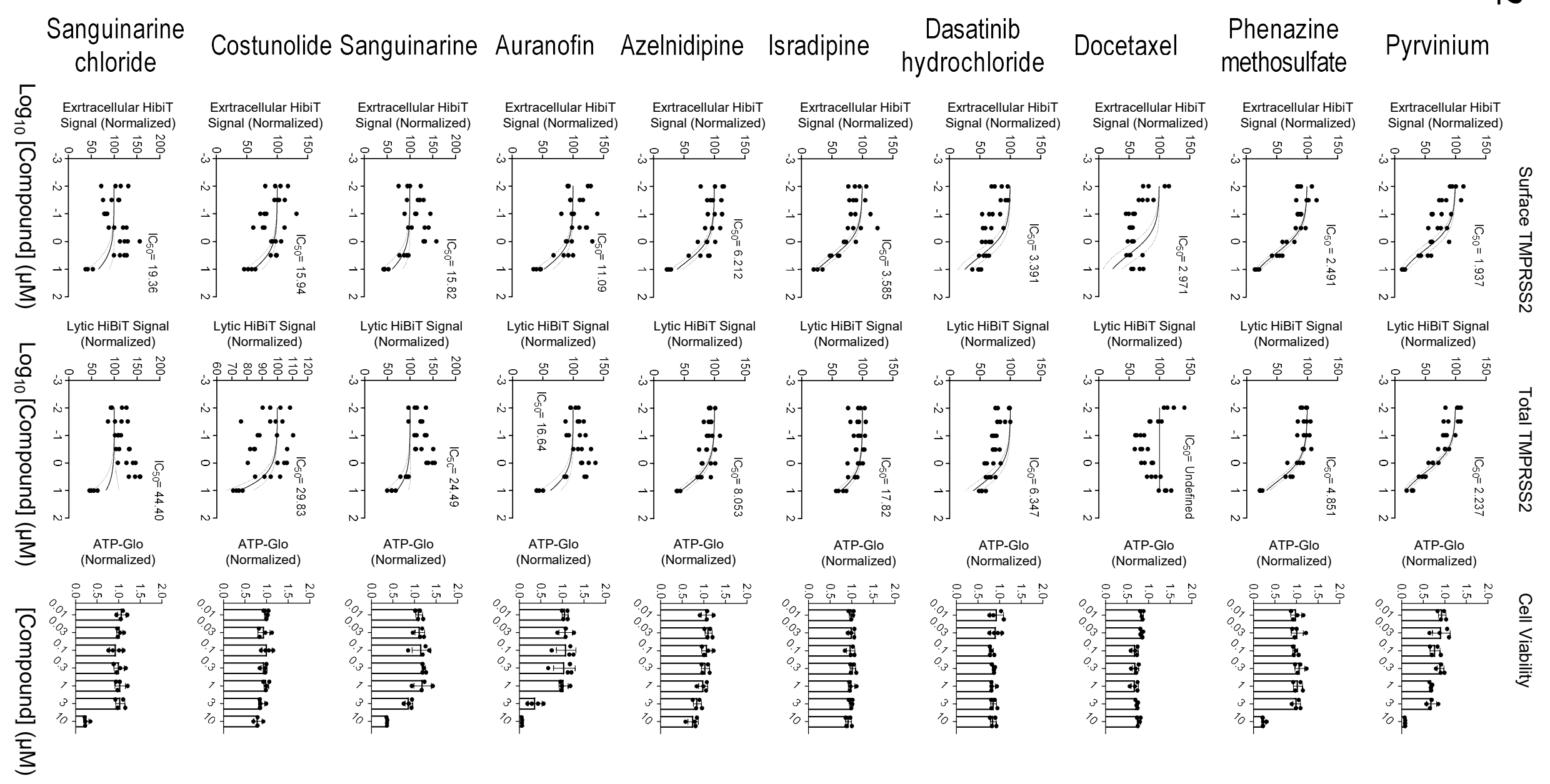


Fig. S3

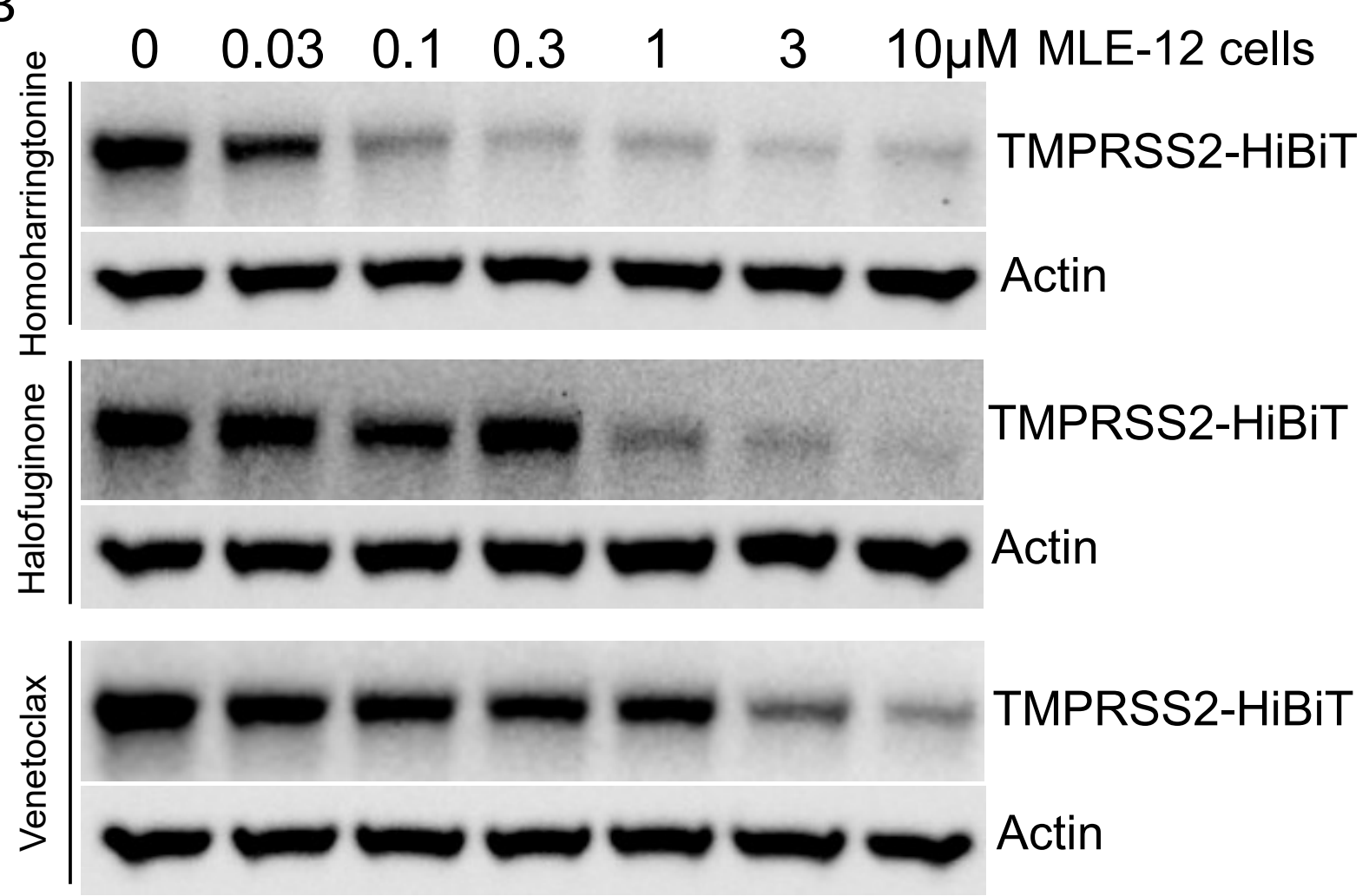


Fig. S4

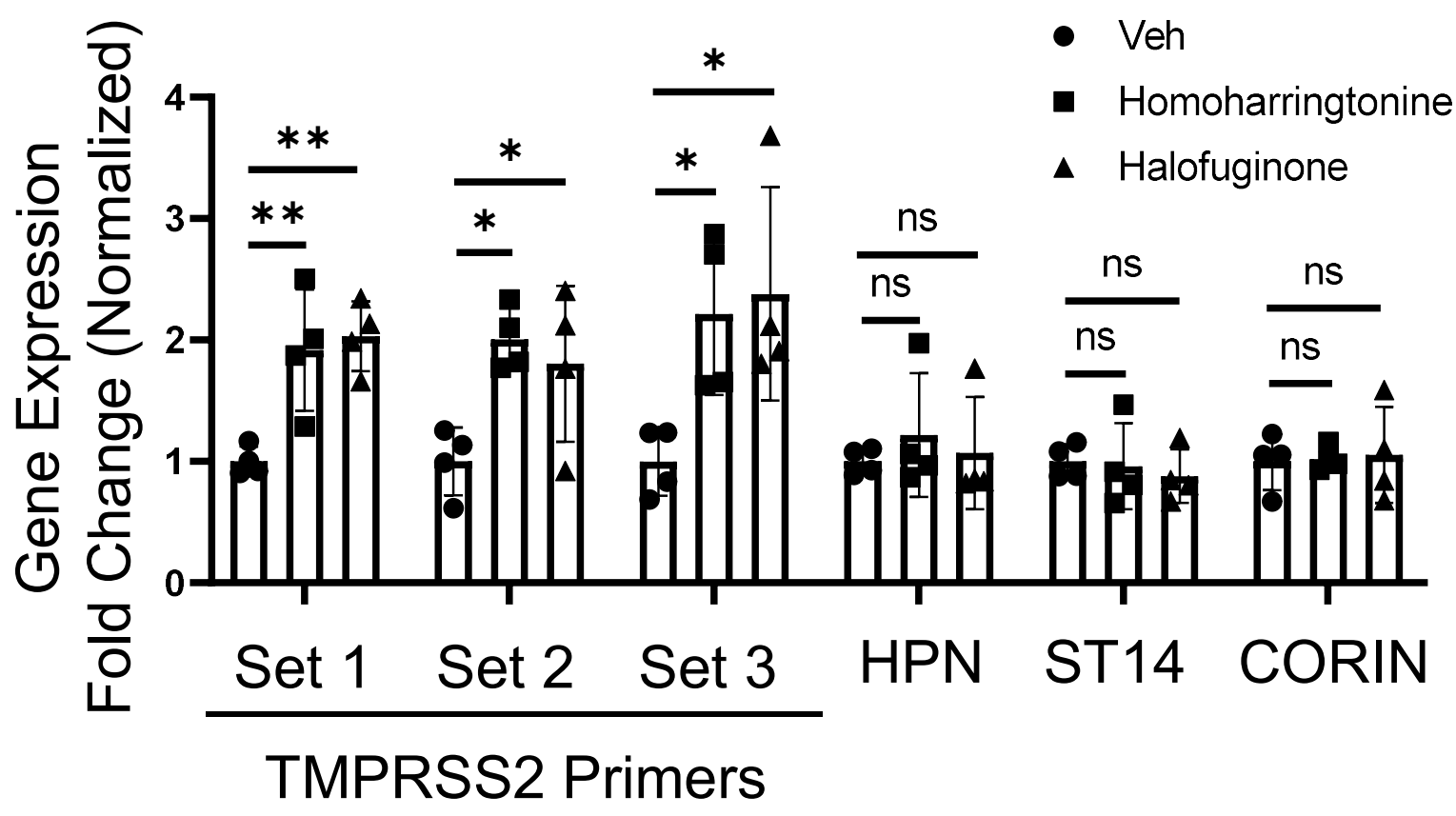


Fig. S5

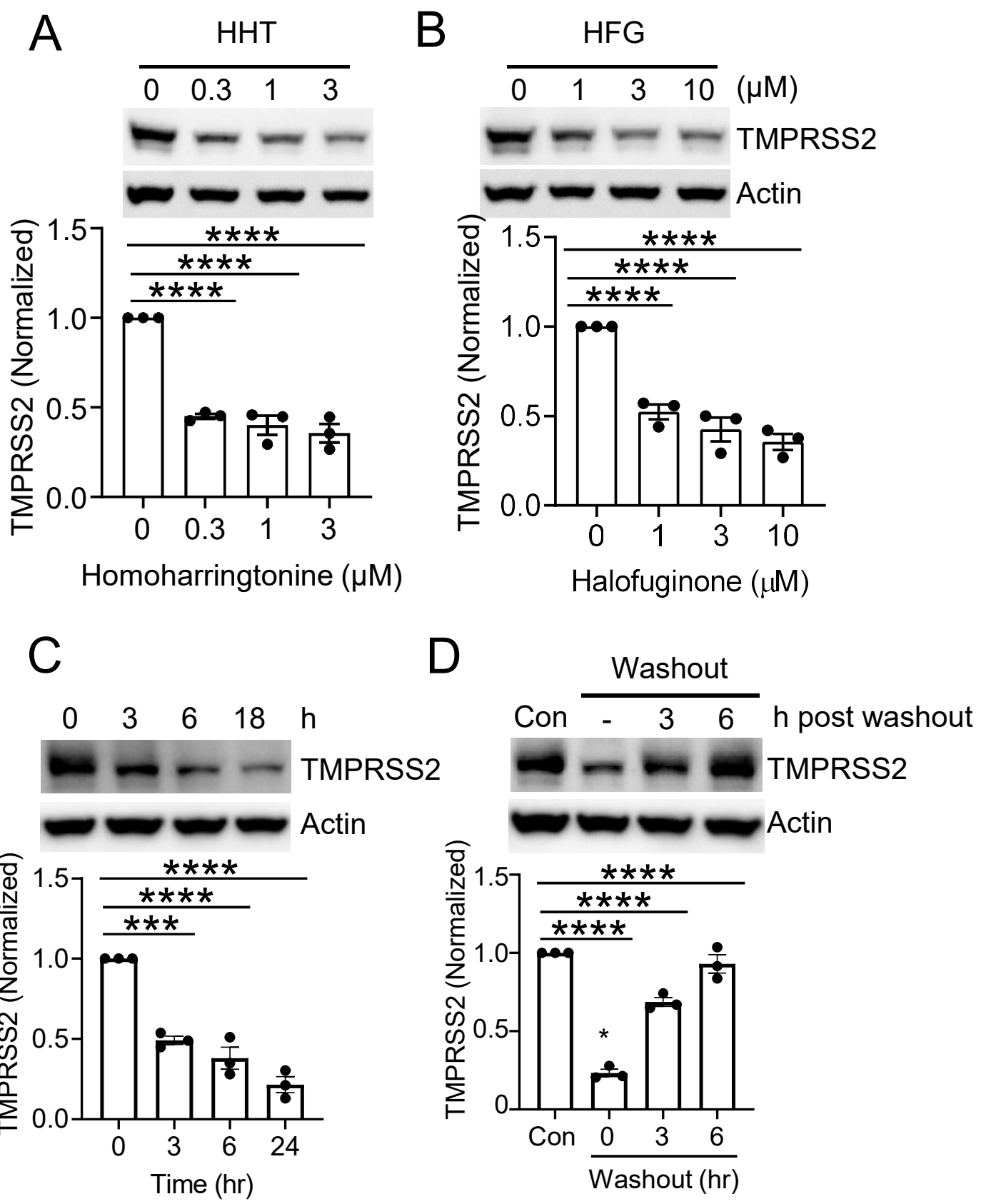


Fig. S6

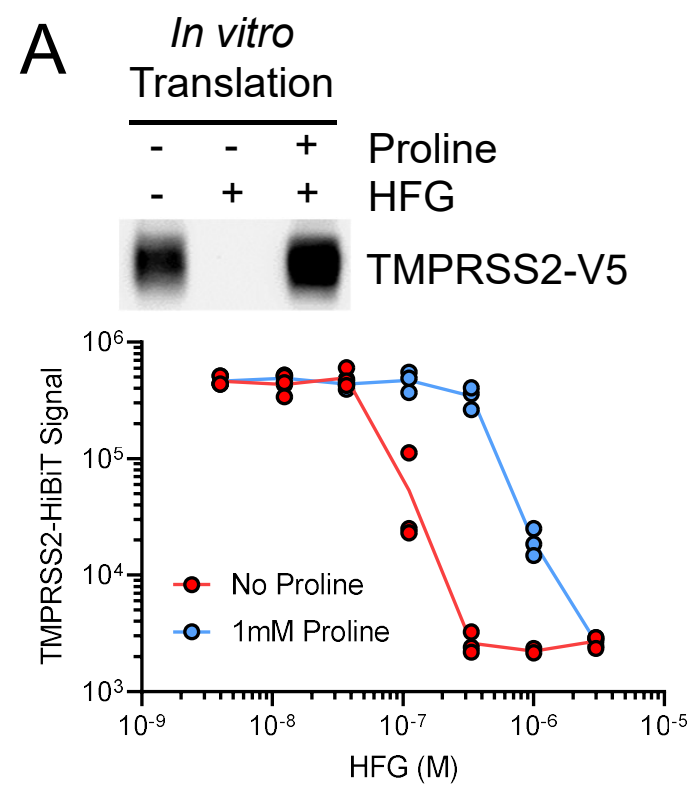

B

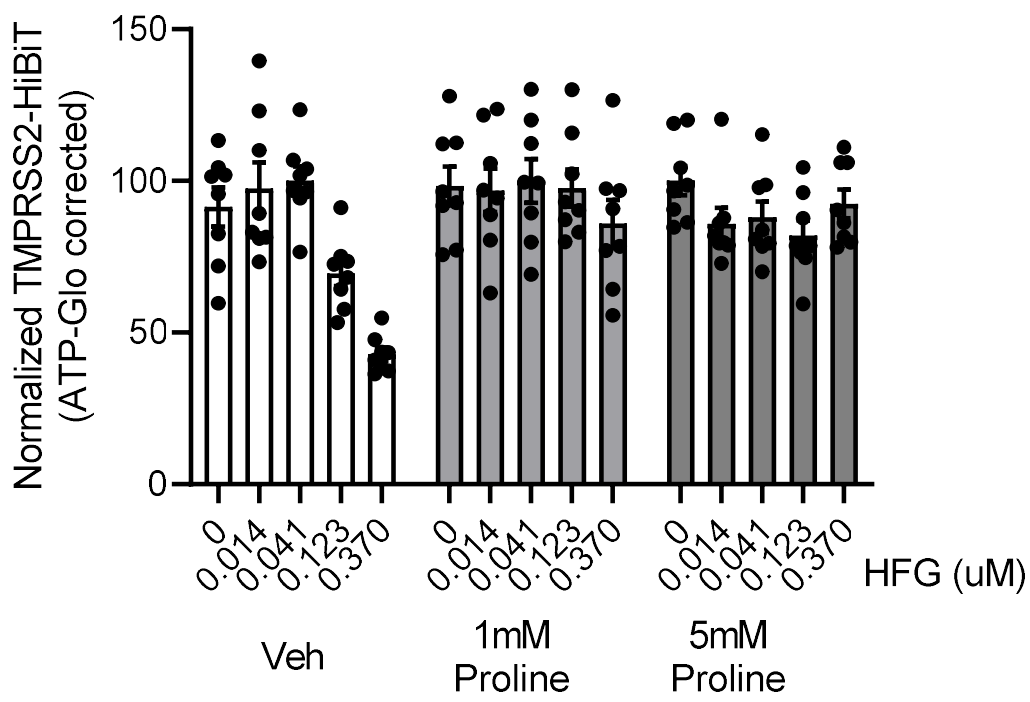


Fig. S7

A

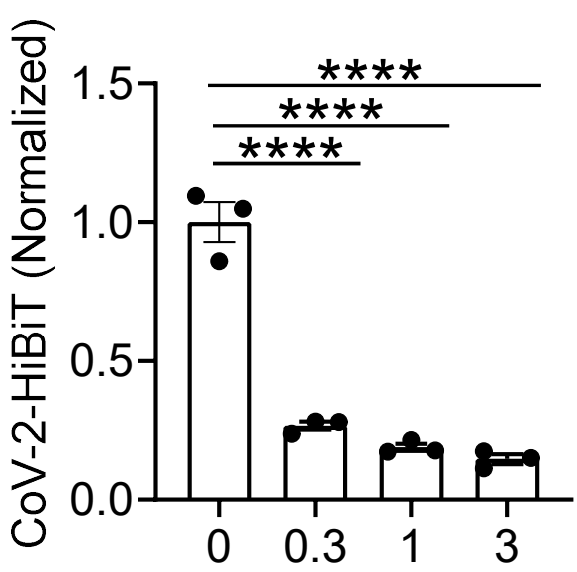

Homoharringtonine $(\mu \mathrm{M})$

C

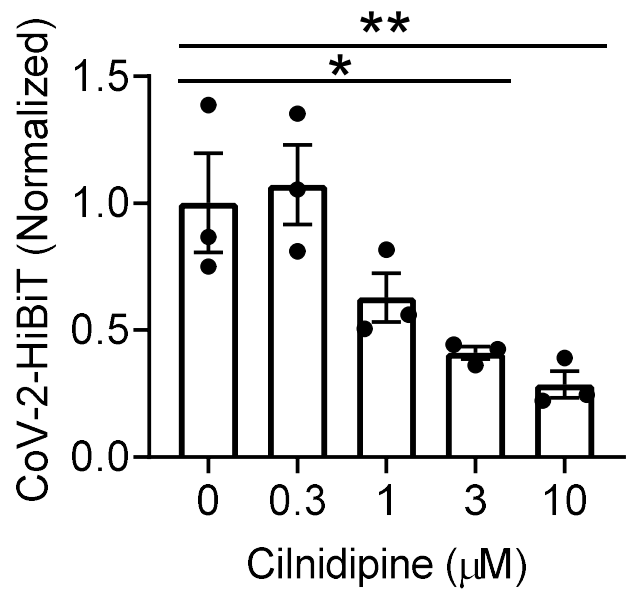

B

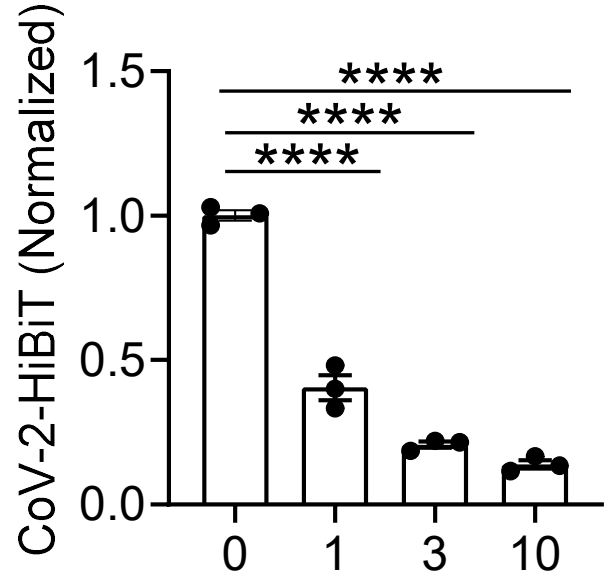

Halofuginone $(\mu \mathrm{M})$

D

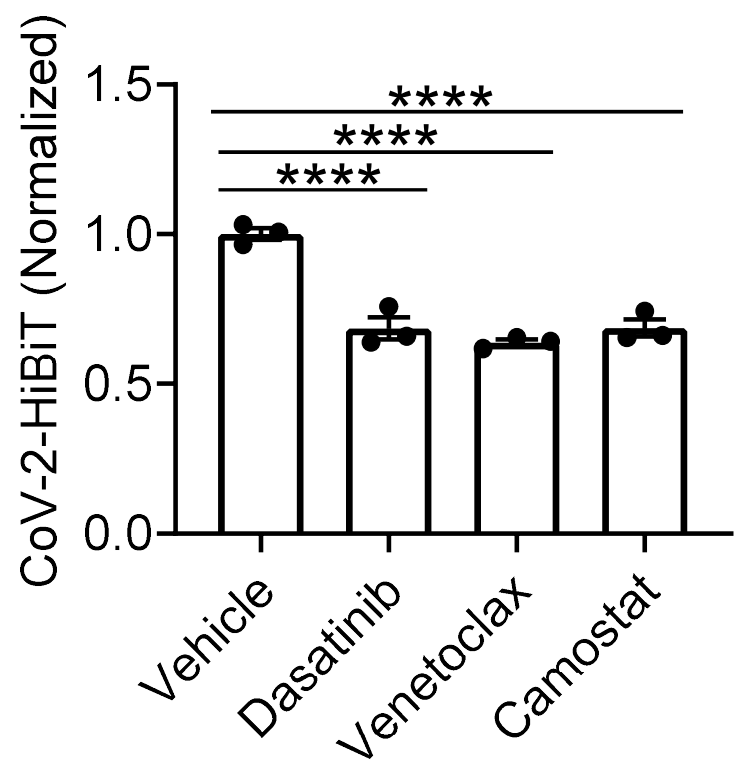




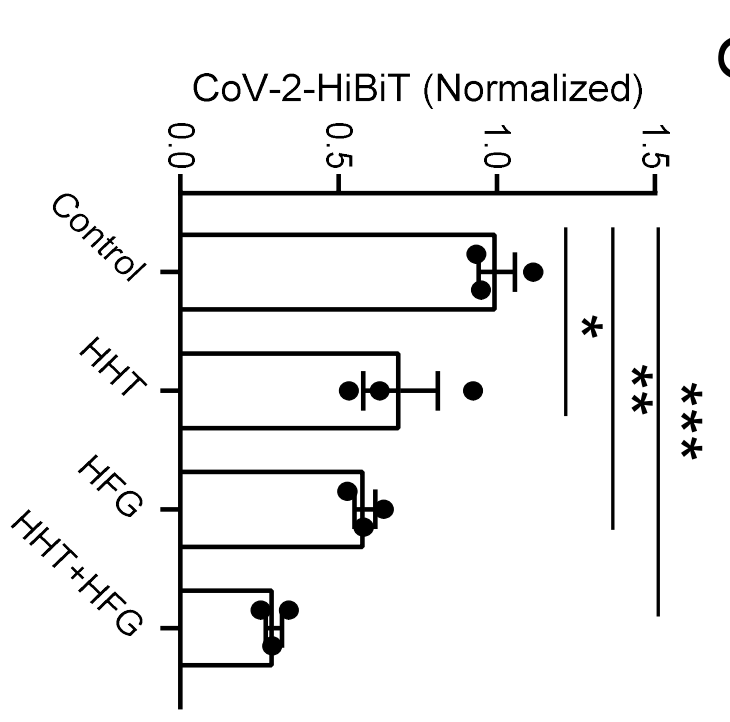

$\Omega$
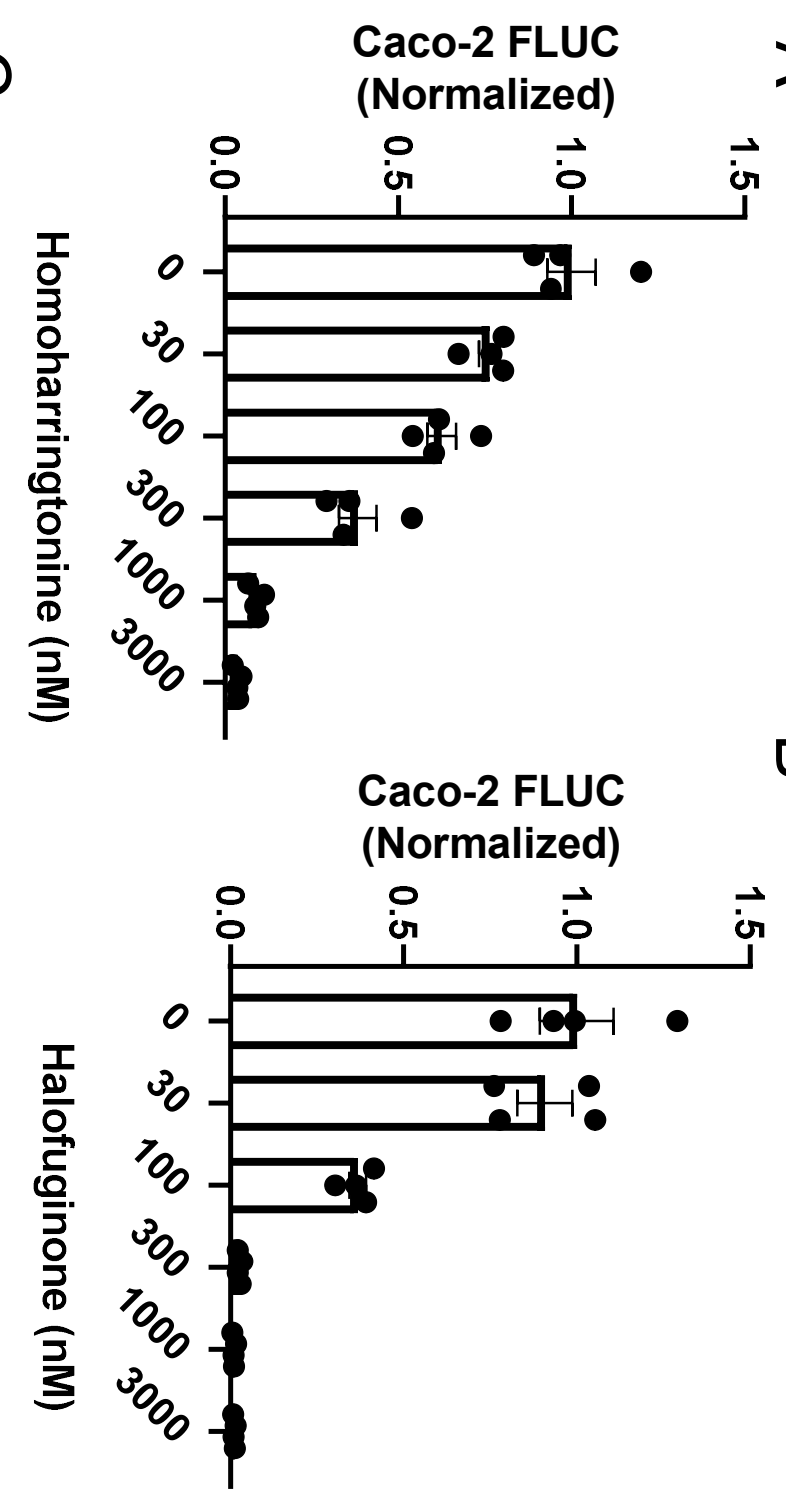
Fig. 59

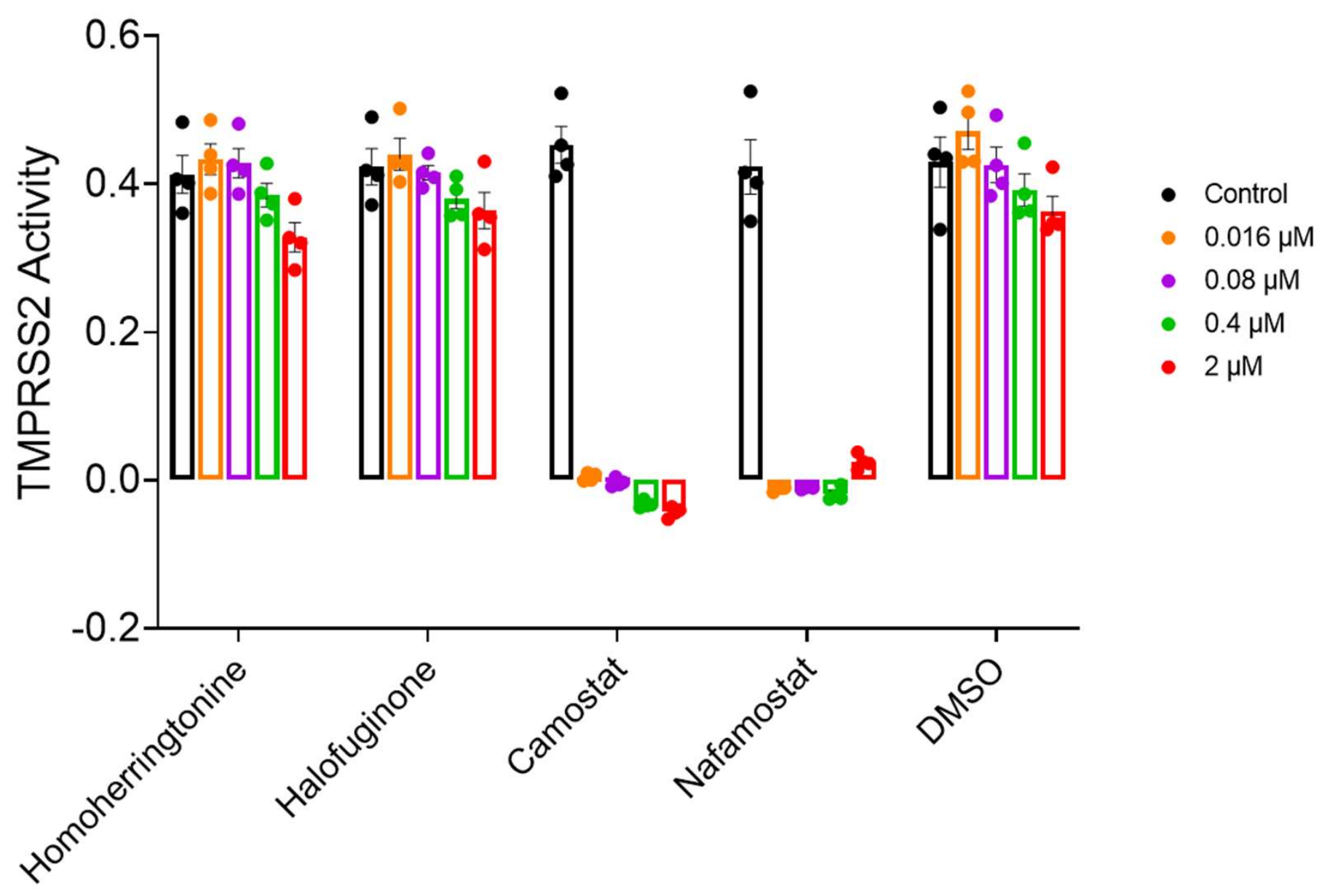


Fig. S10

A

$\begin{array}{lllllllllll}40 & 42 & 44 & 46 & 48 & 50 & 52 & 54 & 56 & 58 & { }^{\circ} \mathrm{C}\end{array}$

Veh

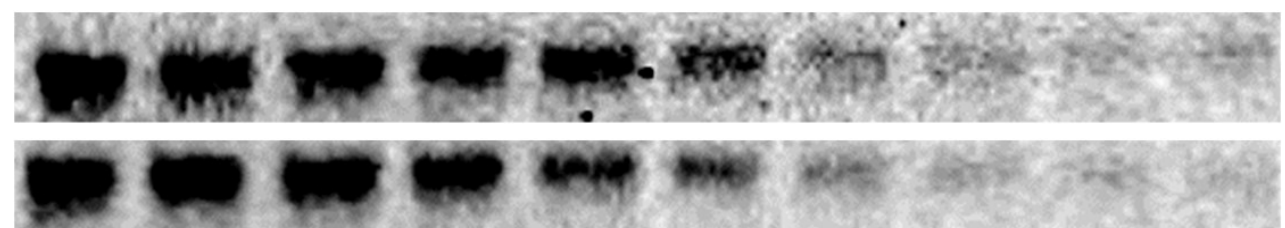

TMPRSS2-HiBiT

HFG

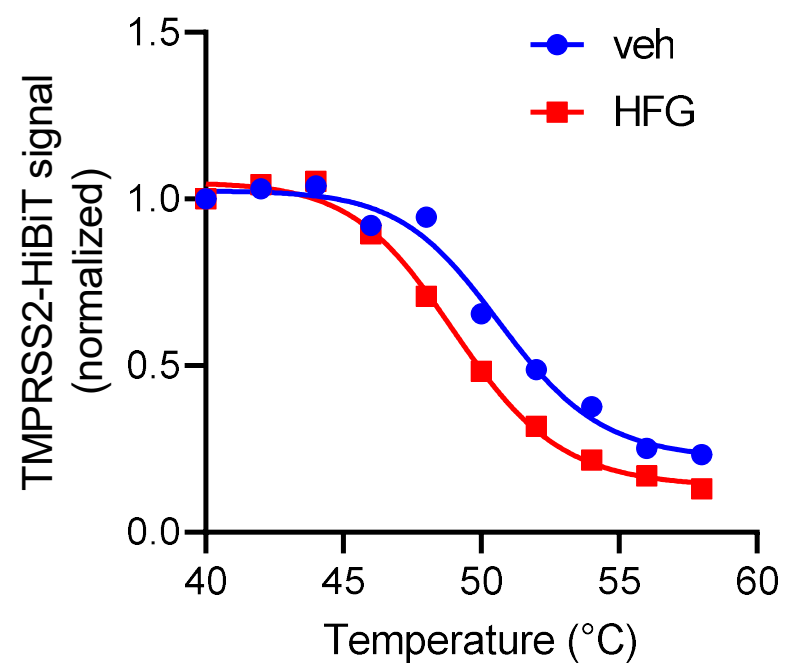

B
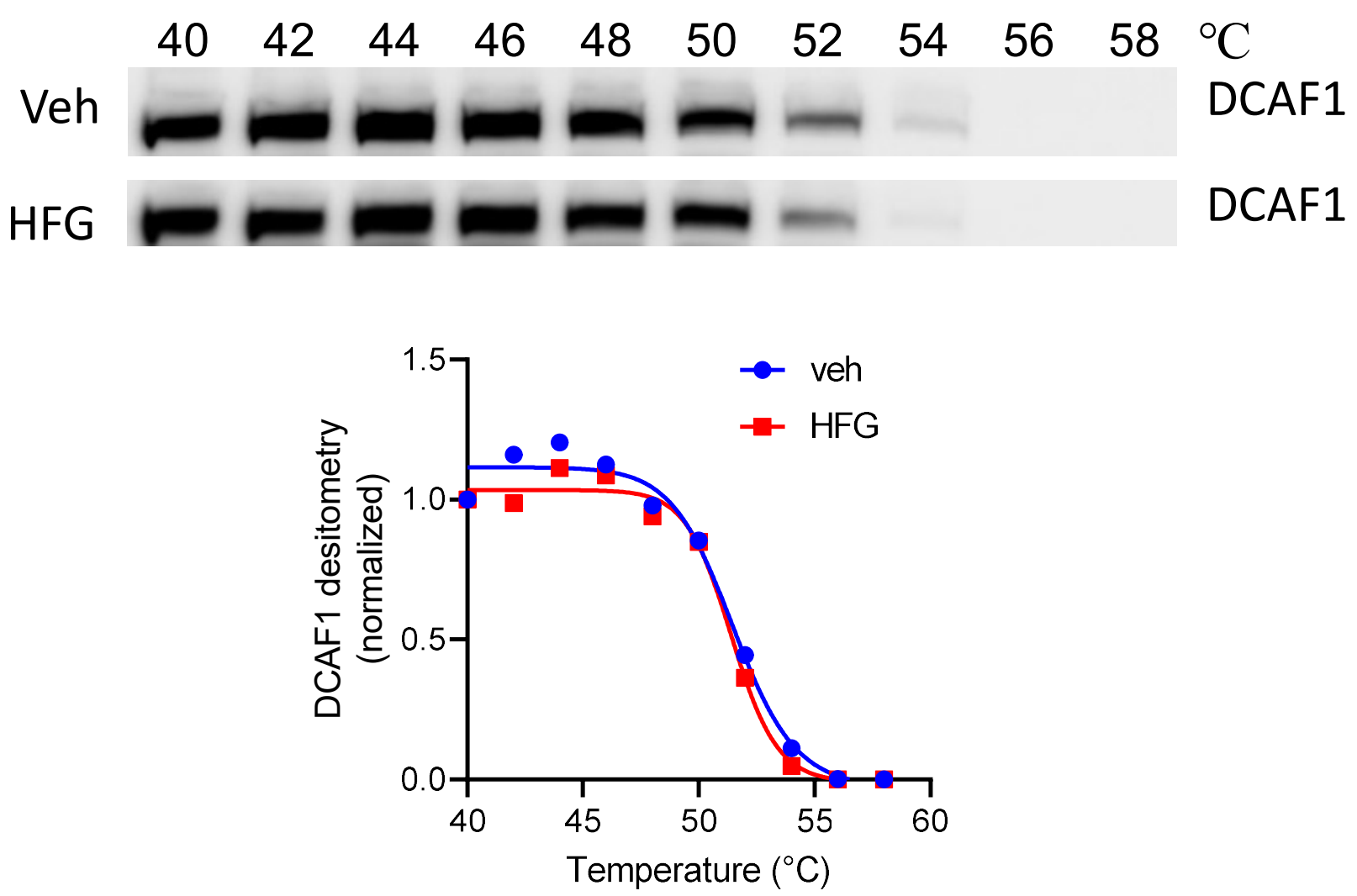
Fig. S11

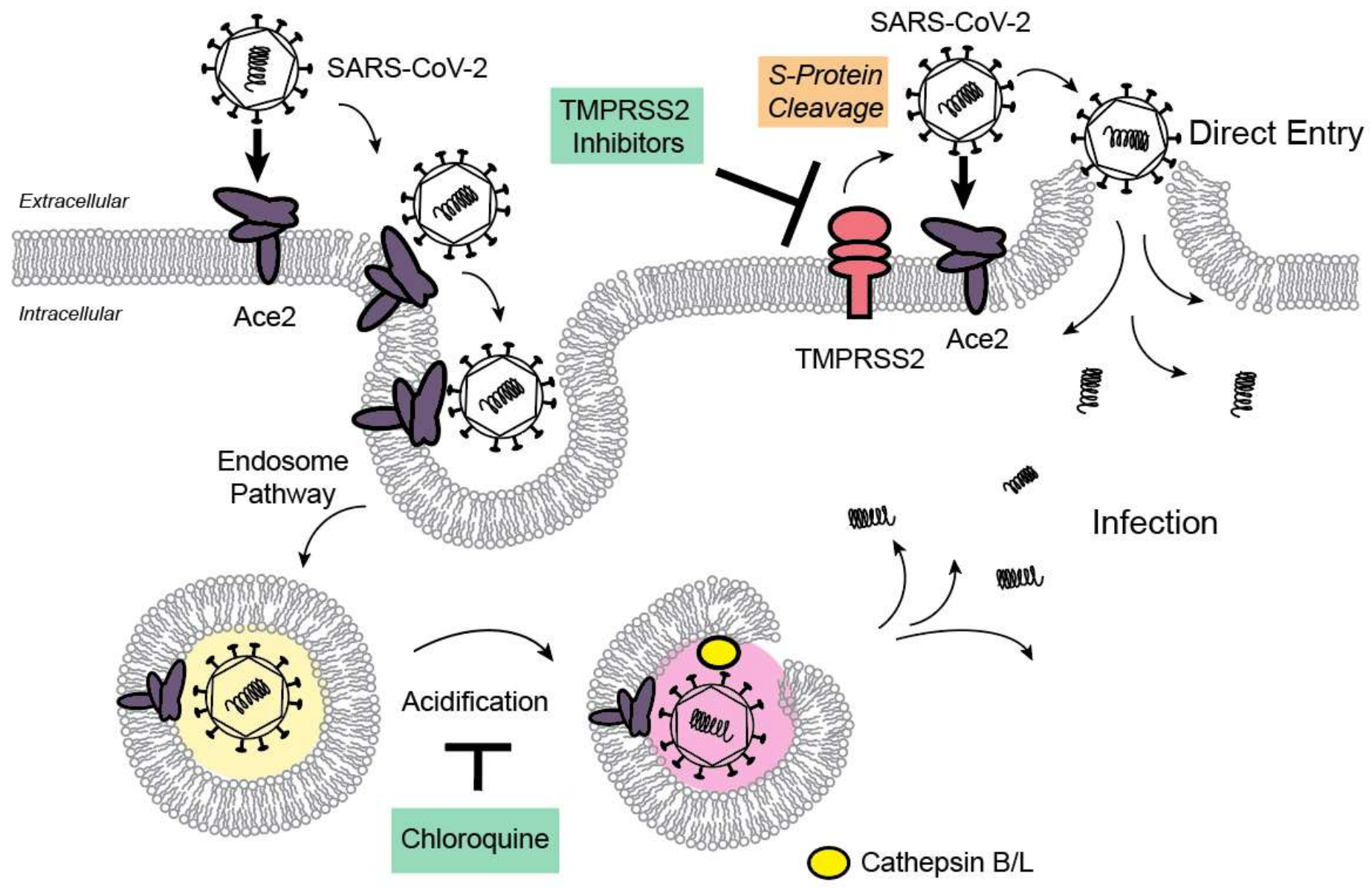




\section{Figures}

A

HiBiT-Nanoluciferase System

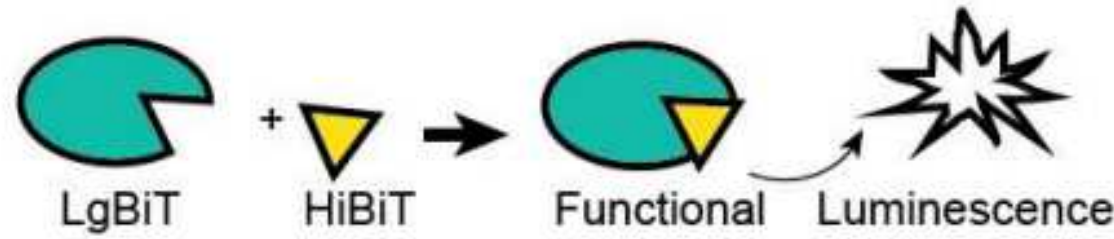

Fragment Fragment Nano-Luciferase

B

Tagging of Essential Protease for SARS-CoV-2 Infectivity

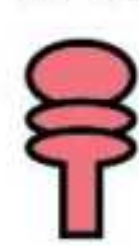

TMPRSS2

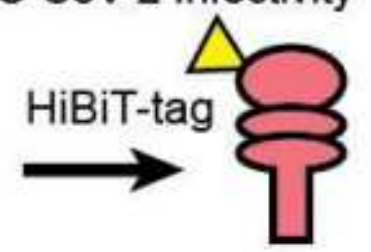

TMPRSS2
C

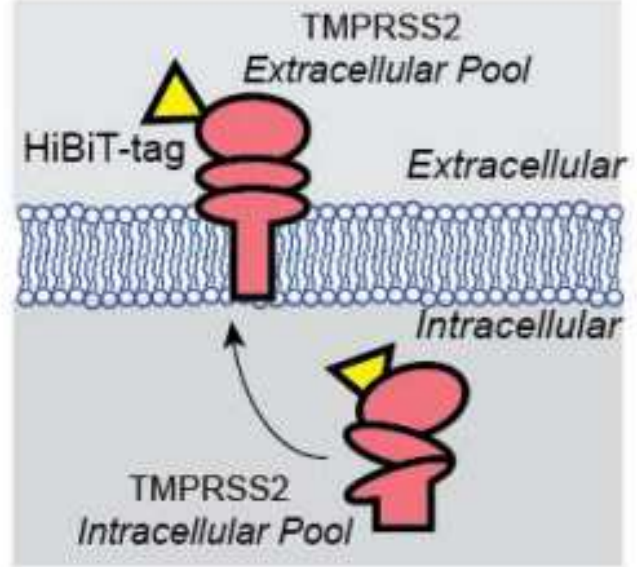

D TMPRSS2-HiBiT Expression in Airway Epithelial Cells

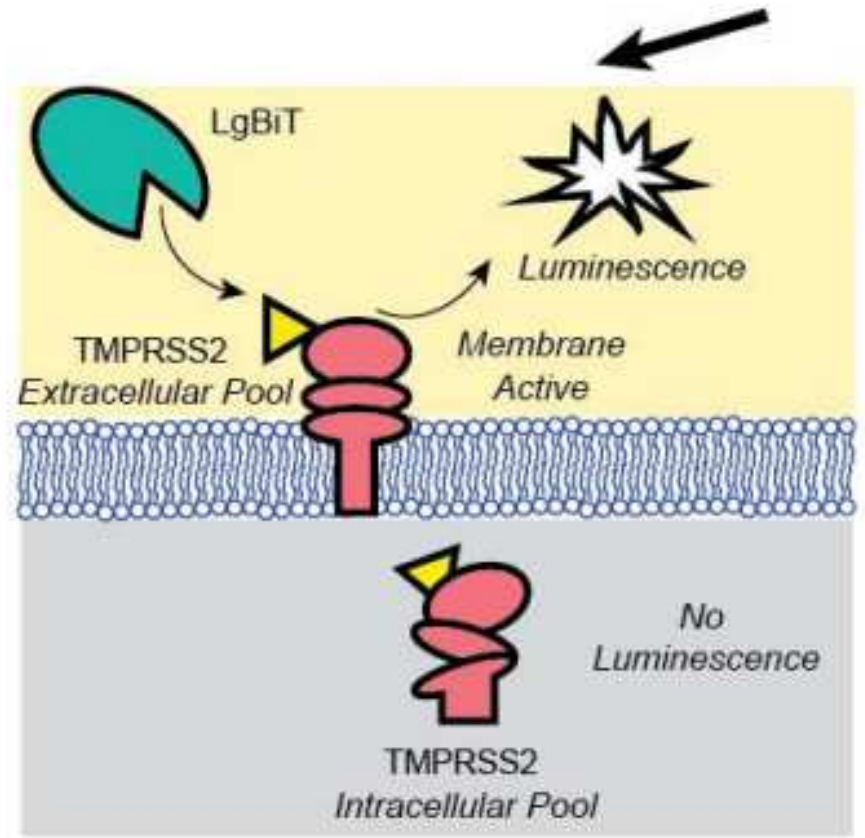

Extracellular HiBiT Detection
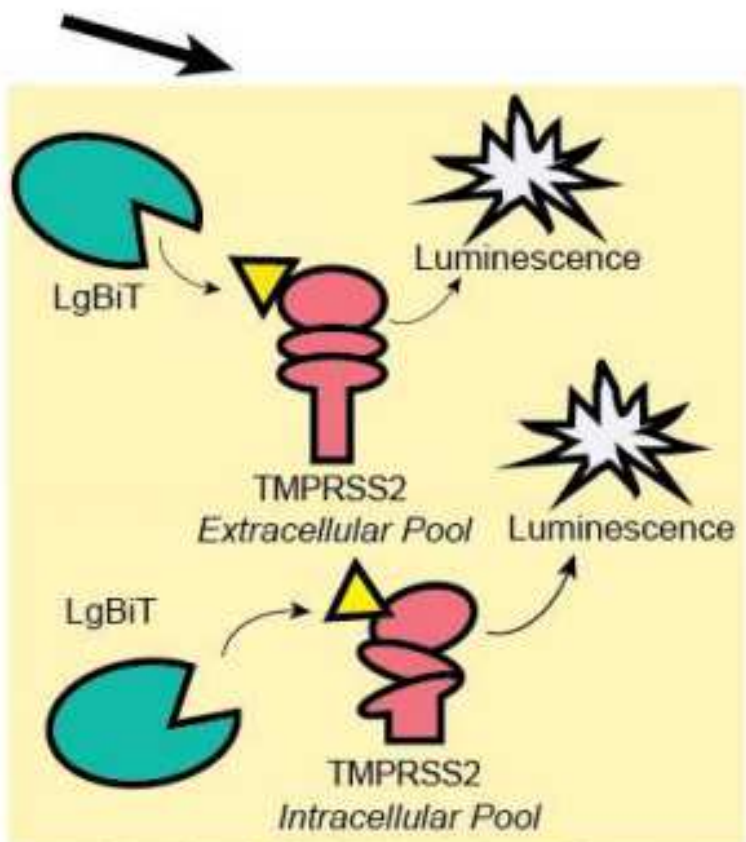

Lytic HiBiT Detection

Figure 1

Schematic of TMPRSS2-HiBiT detection for high throughput screen. (A) The split nano-luciferase components $\mathrm{LgBiT}$ and $\mathrm{HiBiT}$ can interact to form a functional enzyme that generates luminescence. (B) Human TMPRSS 2 cDNA was C-terminally tagged with a HiBiT sequence on a domain that is extracellular 
when TMPRSS2 is present in the plasma membrane. (C) The TMPRSS2-HiBiT construct was expressed in human airway cells where it exists in an intracellular pool and a plasma membrane-associated pool. (D) Non-lytic extracellular HiBiT detection results in LgBiT-HiBiT complementation solely with the pool of plasma membrane localized TMPRSS2. Following extracellular HiBiT detection, cells are lysed and the total TMPRSS2- HiBiT is then quantified.

Fig. 2 A

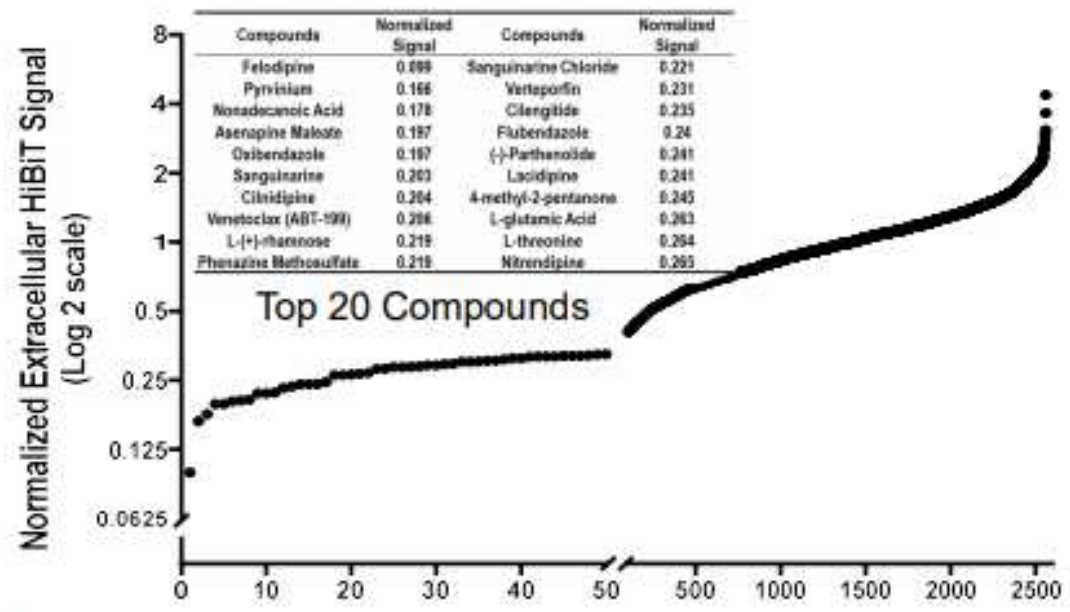

B
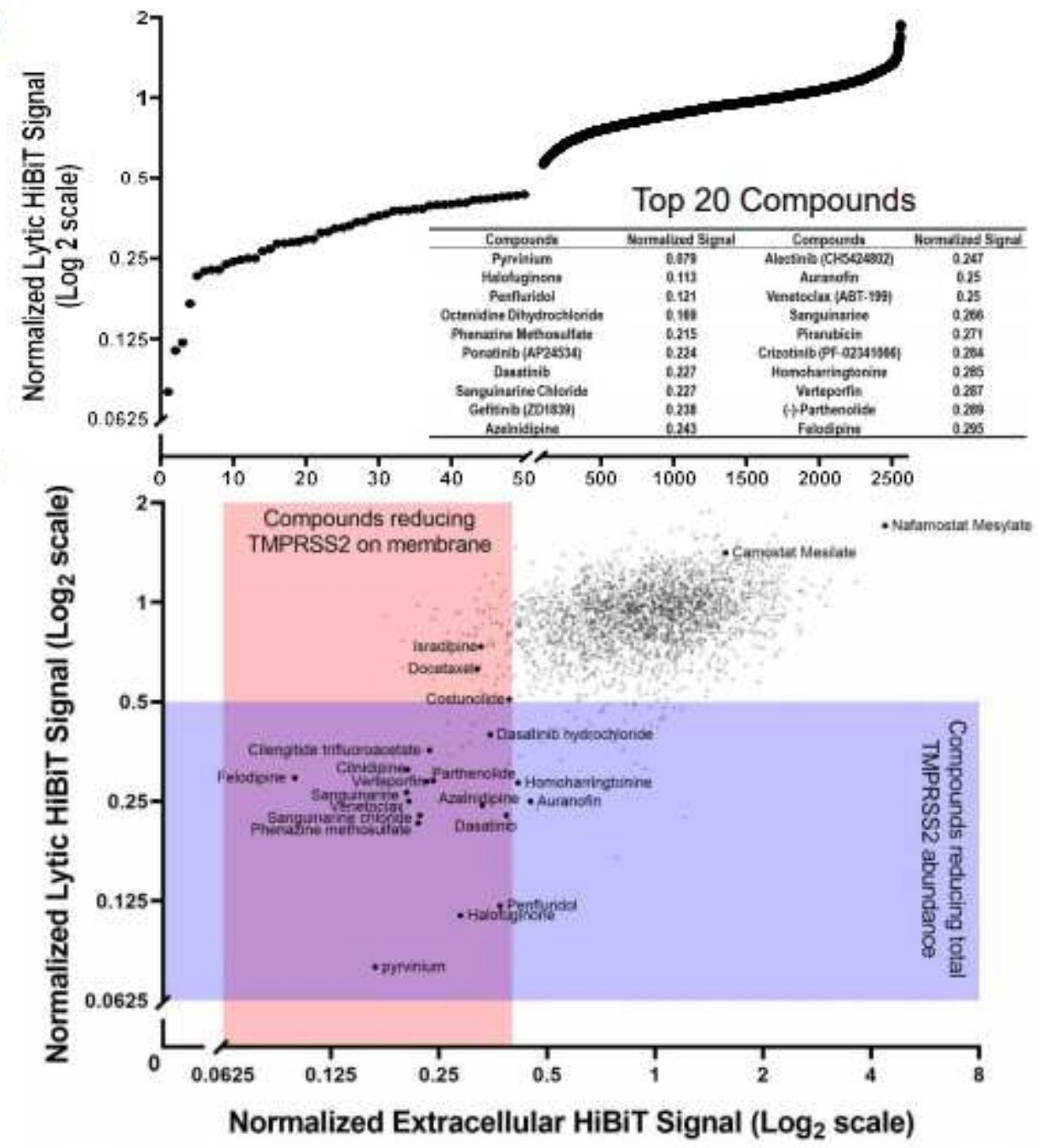

Figure 2 
Screening of airway cells with a FDA-approved or clinically active compound library for agents that reduce TMPRSS2-HiBiT levels. (A) HTS results from the non-lytic extracellular HiBiT detection of TMPRSS2-HiBiT. The top 20 compounds are specified. (B) Lytic HiBiT detection reflecting total cellular TMPRSS2-HiBiT; top 20 compound hits are listed. (C) Scatterplot of hit compounds from both screens. Compounds that reduce membrane TMPRSS2- HiBiT signal (non-lytic extracellular HiBiT detection) are shown in pink, compounds that reduce total TMPRSS2-HiBiT signal (lytic HiBiT detection) are shown in purple. Some individual compounds are specified.

\section{Fig.3 Surface TMPRSS2}
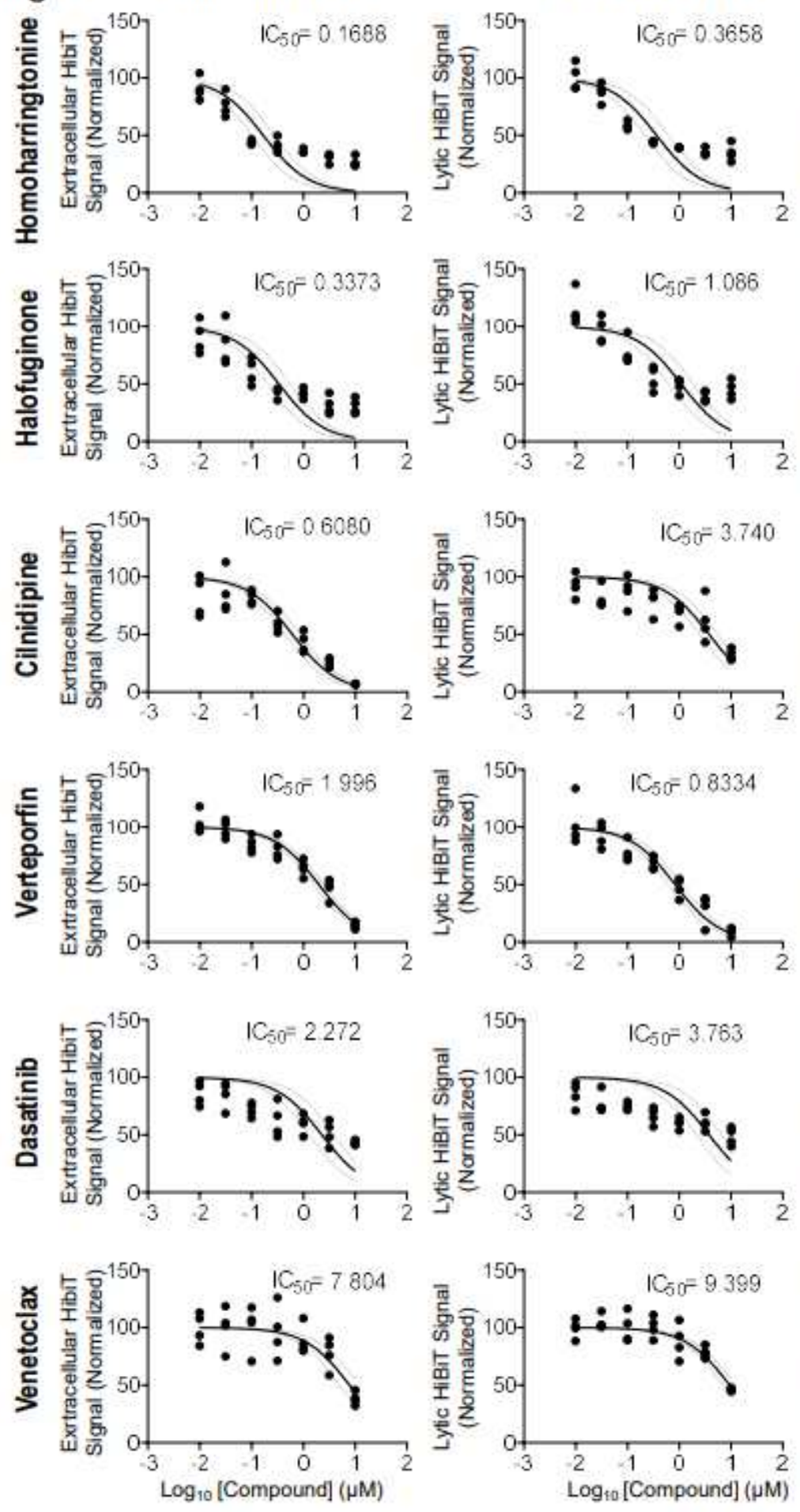

Total TMPRSS2
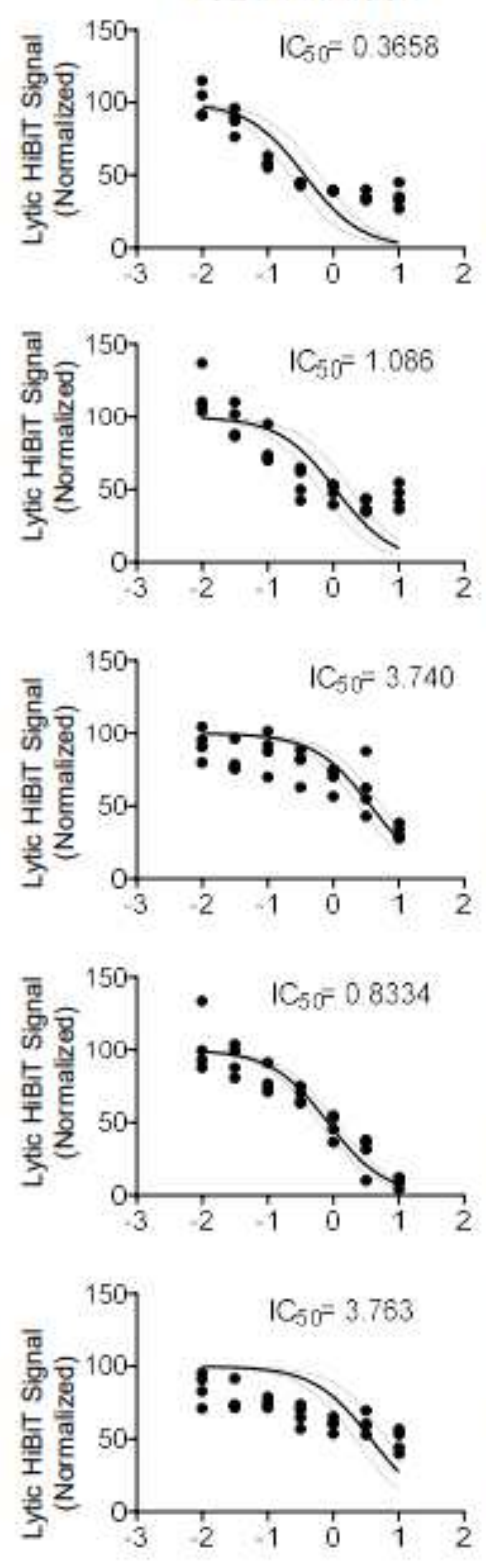

Cell Viability
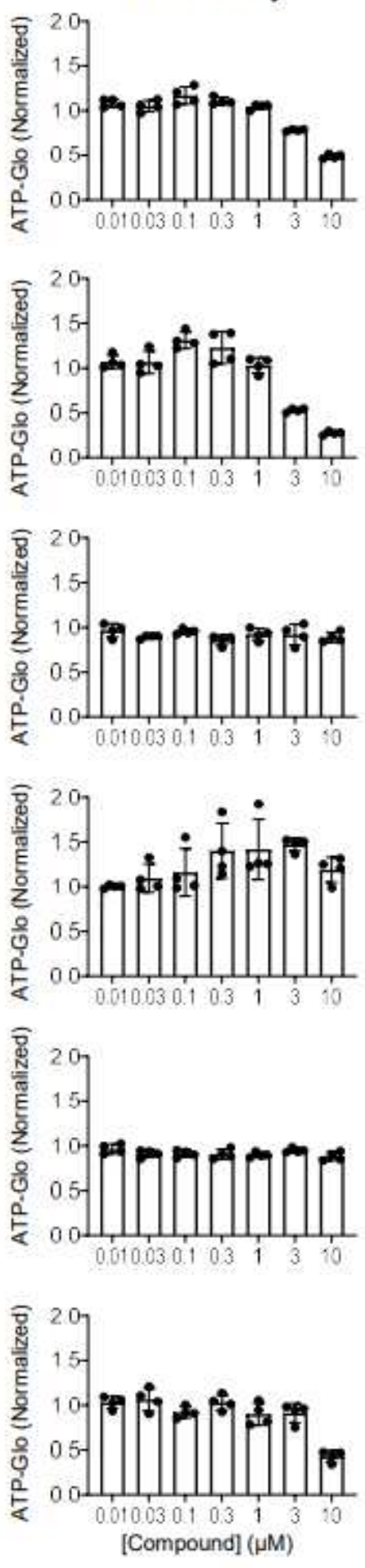


\section{Figure 3}

Determination of the potency for a subset of identified compounds. A selection of six of the most promising drugs were assessed for their activity (IC50) to inhibit TMPRSS2 expression extracellularly (first column) or to inhibit total TMPRSS2 (middle column). An assessment of cellular toxicity for each compound (CellTiter-Glo) is shown in the last column. The remaining activity profiles for other identified agents are found in the supplementary figures. data are mean +/- SEM $(n=4)$.

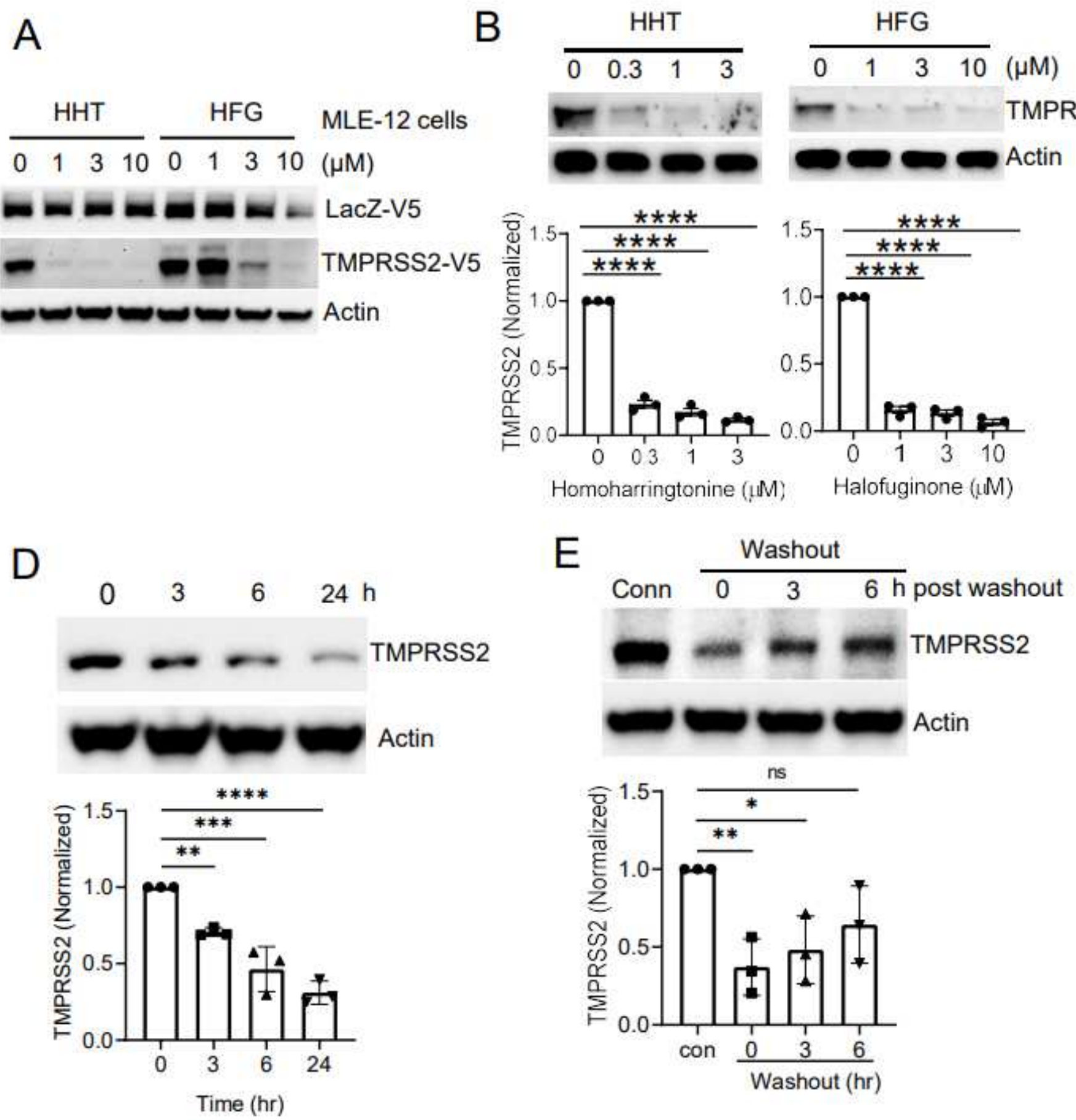

Figure 4 
Homoharringtonine and halofuginone potently reduce TMPRSS2 protein levels. (A) Immunoblot data from MLE-12 cells co-expressing LacZ-V5 and TMPRSS2-V5 treated with homoharringtonine (HHT) or halofuginone (HFG) at the indicated concentrations for $18 \mathrm{hr}$. (B-C) Immunoblot analysis of endogenous TMPRSS2 protein level in Caco-2 cells treated for $18 \mathrm{hr}$ with HHT (B) or HFG (C). TMPRSS2 densitometry is shown, data are mean +/- SEM ( $n=3)$. (D) Timecourse treatment of HFG-treated Caco-2 cells $(3 \mu \mathrm{M})$. TMPRSS2 densitometry is shown, data are mean +/- SEM ( $n=3)$. (E) Immunoblot analysis of Caco-2 cells treated with HFG for $18 \mathrm{hr}$ prior to removing the drug, adding fresh media, and then analyzing the protein recovery time course. (All TMPRSS2 densitometry that is shown represents mean $+/-\operatorname{SEM}(n=3)$. Actin is

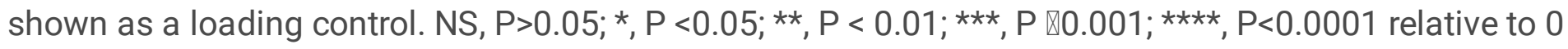
time point or control, or as indicated by one-way ANOVA with Dunnett's test of multiple comparisons (BE). 
A

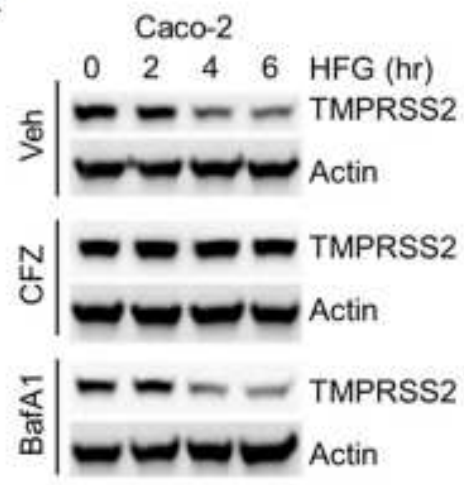

C

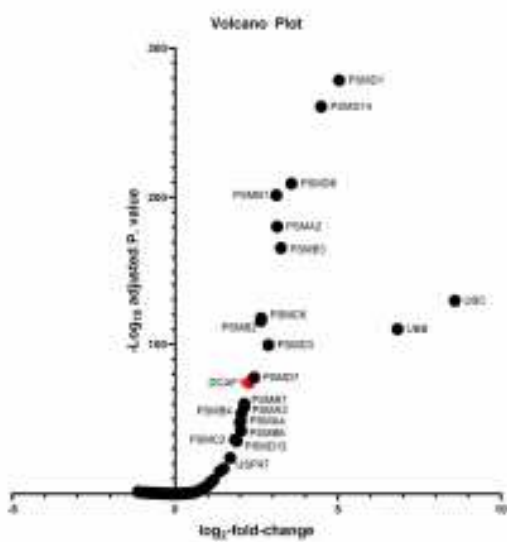

F
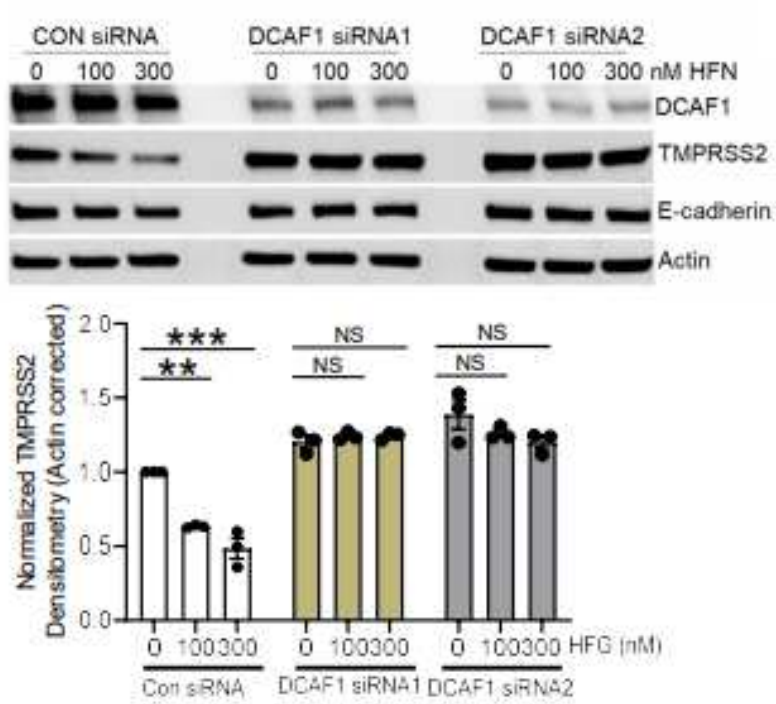

B

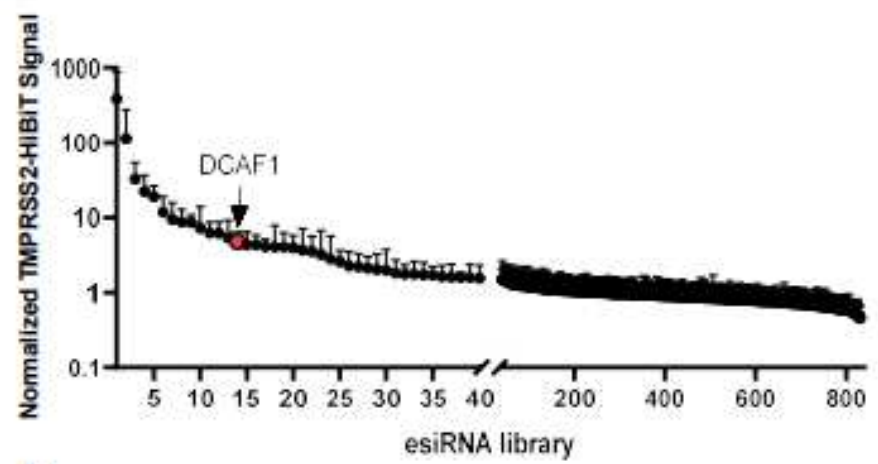

D

E HA-DCAF1(ug)

- + HA-DCAF1

+ + TMPRSS2-HIS-V5

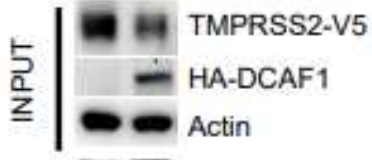

$\begin{array}{lll}0 & 1 & 2\end{array}$

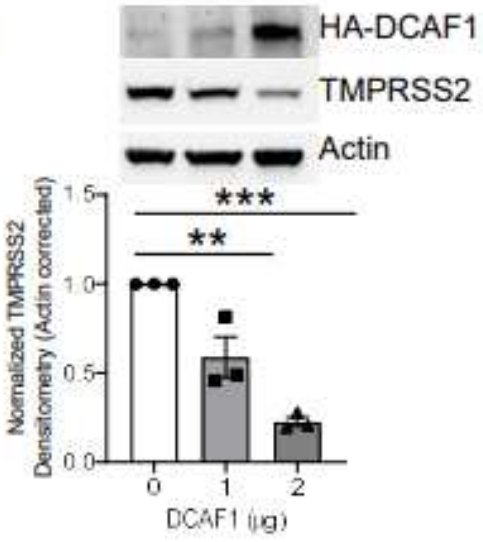

Fig.5

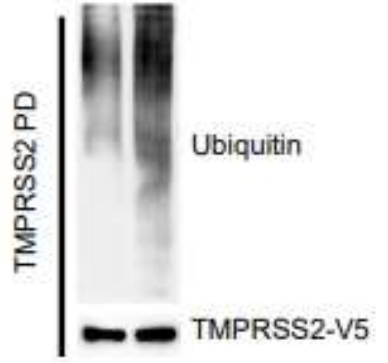

G

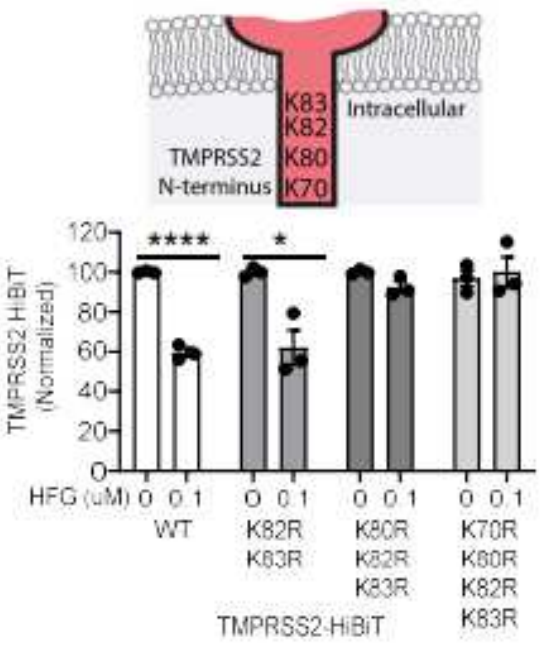

\section{Figure 5}

TMPRSS2 is degraded through ubiquitin proteasome system, which is required for HFG efficacy. (A) Immunoblotting assay of Caco-2 cells treated with HFG in combination with carfilzomib (CFZ) or bafilomycin A1 (BafA1) and probed for TMPRSS2. (B-C) TMPRSS2- HiBiT signal was measured following siRNA knockdown of ubiquitination-related machinery. (B) Ubiquitination siRNA library screening results ordered by increase in TMPRSS2-HiBiT signal. The E3 ligase DCAF1 was detected as a top hit. (C). 
Volcano plot of TMPRSS2-HiBiT signal screening with Ubiquitination siRNA library from $n=3$ independent screening assays. Statistical significance is plotted against log2-fold change in TMPRSS2-HiBiT signal. Top hits are annotated. (D) DCAF1 co-expression increases TMPRSS2 ubiquitination. (E) Immunoblot analysis of Beas2B cells with increasing expression of DCAF1, TMPRSS2 densitometry shown represents mean + /- SEM $(n=3)$. ( $F)$ Immunoblot analysis of Beas-2B cells with control or DCAF1 siRNA treatment followed by HFG treatment. TMPRSS2 densitometry represent mean -/+SEM $(n=3)$. (G) TMPRSS2 intracellular lysines were assayed for their responsiveness to HFG, data represent mean +/- SEM $(n=3)$. Actin is shown as a loading control throughout, and E-cadherin is added as an additional plasma membrane loading control. NS, $\mathrm{P}>0.05 ; *, \mathrm{P}<0.05 ; * \star, \mathrm{P}<0.01 * \star \star, \mathrm{P} \otimes 0.001 ; * \star \star \star, P<0.0001$ relative to control, or as indicated by one-way ANOVA with Dunnett's test of multiple comparisons (E-F), or two-way unpaired t-test $(G)$. 
Fig.6

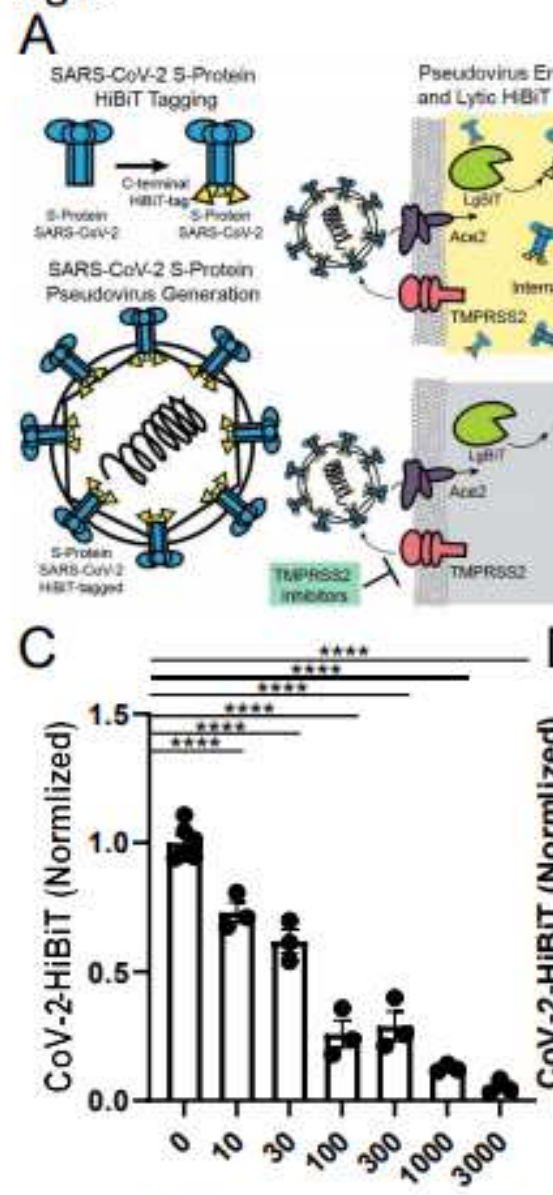

Homoharringtonine $(\mathrm{nM})$

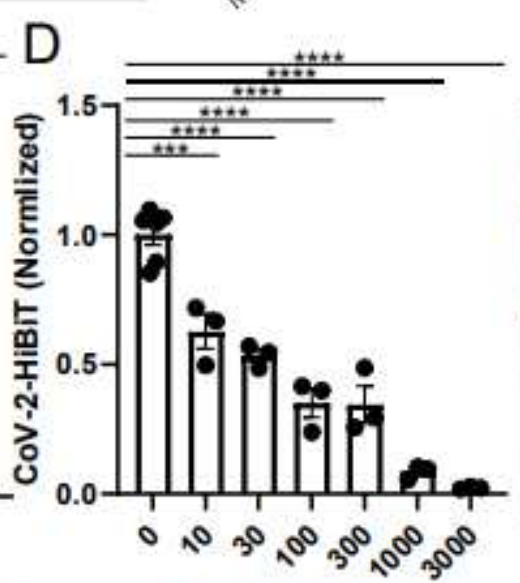

Halofuginone (nM)

$\mathrm{F}$
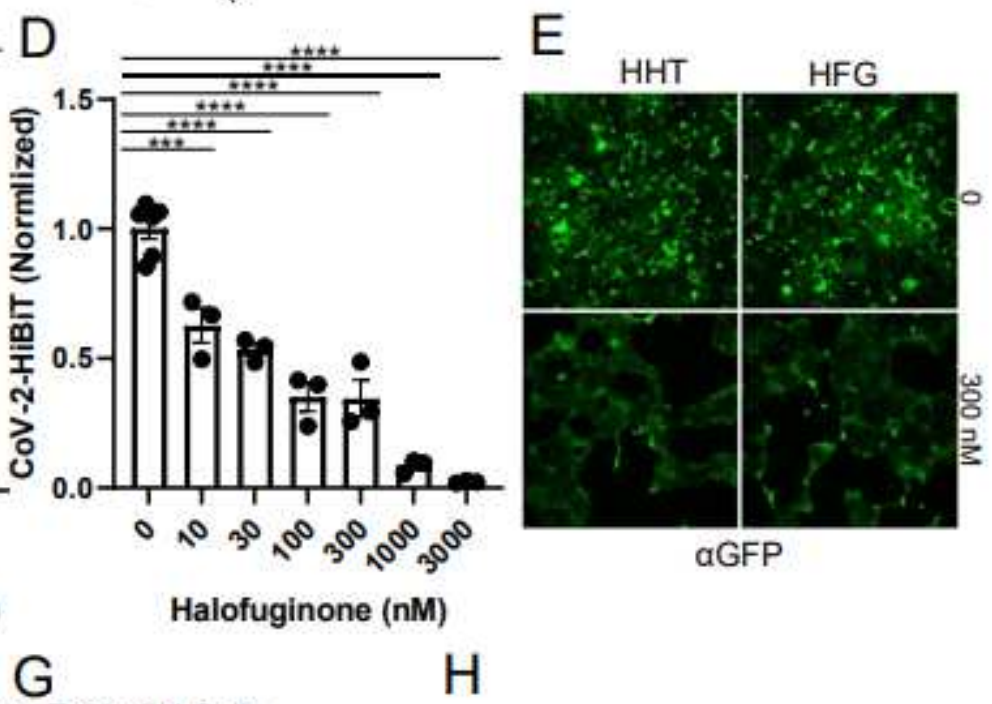

$\mathrm{H}$

Primary Human Airway Epithelial Cells
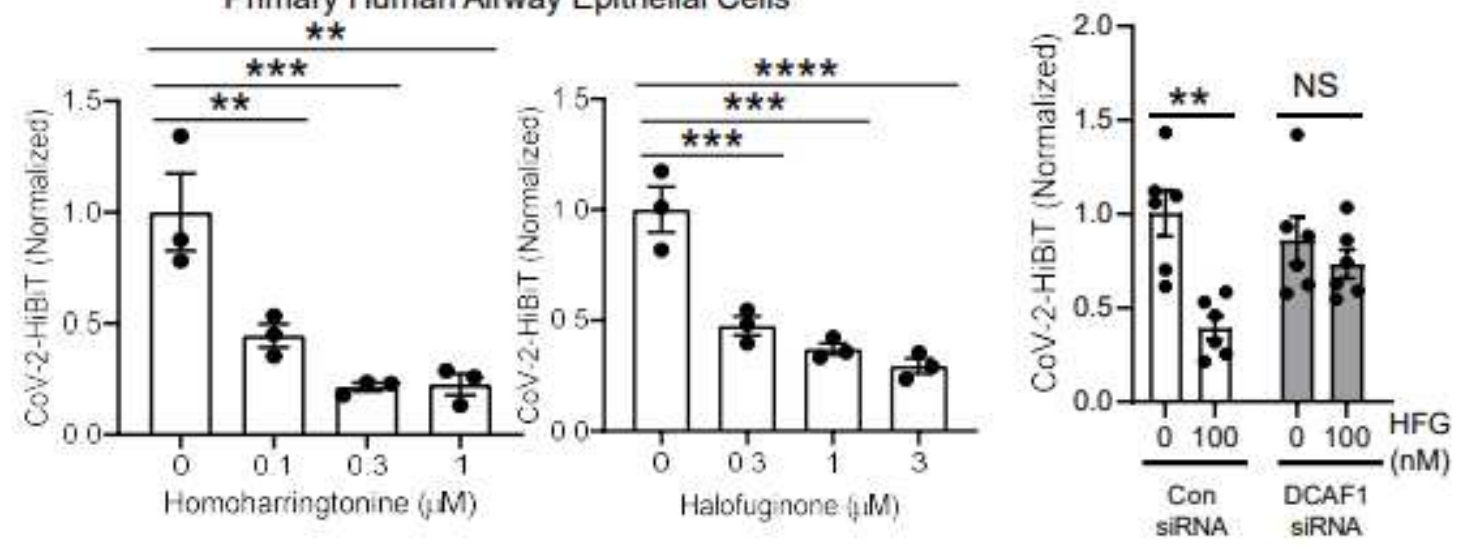

Figure 6

Agents that reduce TMPRSS2 expression markedly inhibit SARS-CoV-2 pseudoviral infection. (A) Schematic of pseudoviral construction and assay. The S protein of SARS-CoV-2 was C-terminally tagged with HiBiT. (B) Level of viral transduction in various cell lines plotted on a logarithmic scale. Calu-3 and Caco-2 cells had the highest observed rates of infection. (C-D) Effects of increasing concentrations of homoharringtonine (HHT) (C) or halofuginone (HFG) (D) on SARS-CoV-2 pseudoviral infection. (E) Effects of HHT or HFG on pseudoviral-mediated GFP expression, determined by immunostaining. (F-G) SARS- 
CoV-2 pseudoviral infection of primary human bronchial epithelial cells in the presence of increasing concentrations of HHT (F) and HFG (G). (H) SARS-CoV-2 pseudoviral infection of Caco-2 cells transfected with DCAF1 siRNA along with HFG treatment (100nM). All SARS-CoV-2 pseudoviral data is corrected to cell number as determined by CellTiter-Glo. **, $\mathrm{P}<0.01$; $* \star \star, \mathrm{P} \otimes 0.001$; $* \star \star \star, \mathrm{P}<0.0001$ relative to 0 time point or control, or as indicated by one-way ANOVA with Dunnett's test of multiple comparisons (F-G), or a twoway ANOVA with Tukey's test of multiple comparisons $(\mathrm{H})$ 